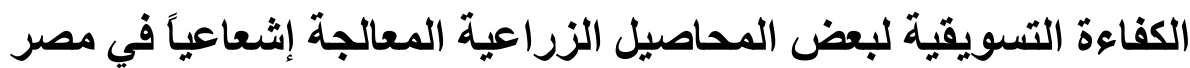

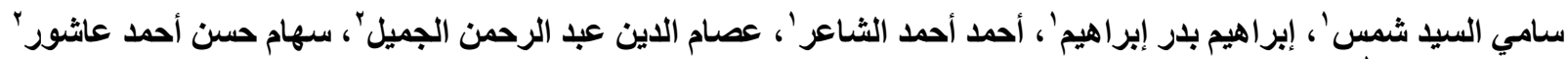

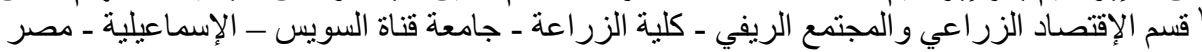

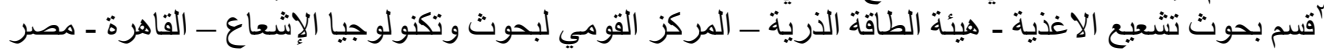

الملخص: تم في هذه الدراسة در اسة أثر استخدام تكنولوجيا التشعيع في تحسين الكفاءة التسويقية لبعص الحاصلات الزر اعية الغذائية وهي

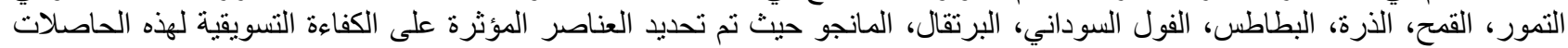

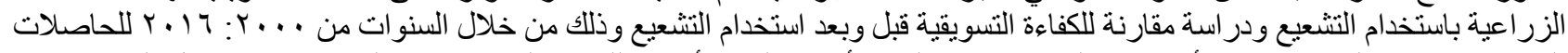

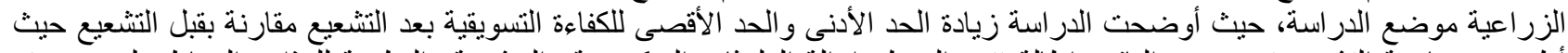

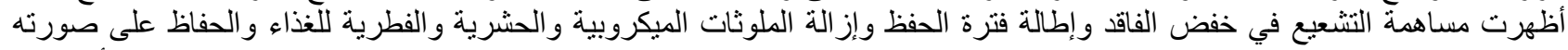

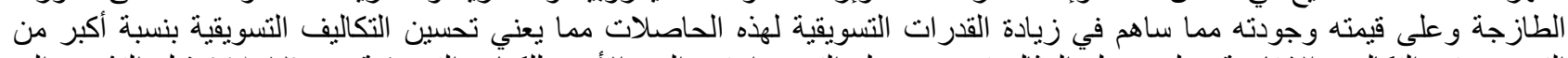

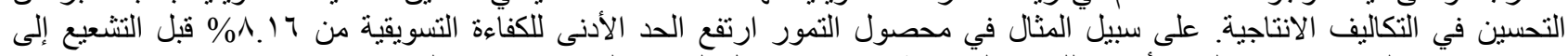

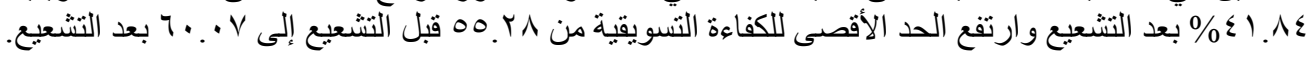
الكلمات الدالة: الفاقد الغذائى، التحليل المالى، العوائد القومية، المعجل الإلكتروني، الاتجاه الزمنى.

r- مقارنة الكفاءة التسويقية للمحاصيل السابقة قبل وبعد استخدام

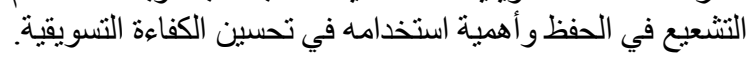
الطريقة البحثية ومصادر البيانات:

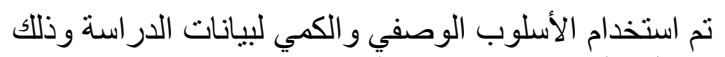

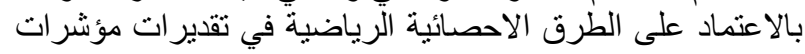

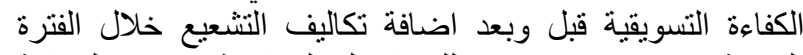

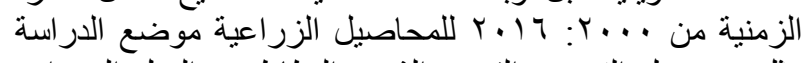

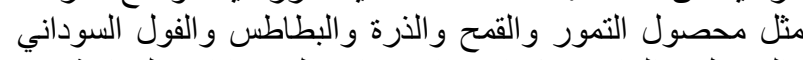

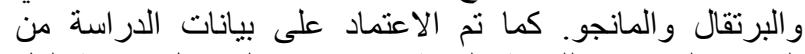

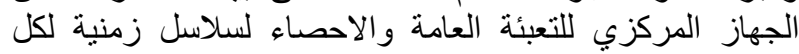

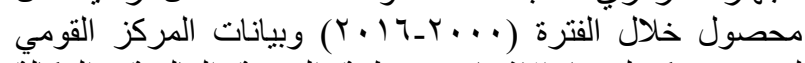
لبحوث وتكنولوجيا الإشعاع ومنظمة الصحة الهُ العالمية والوكالة ولئة الدولية للطاقة الذرية.

\section{نتائج الاراسة}

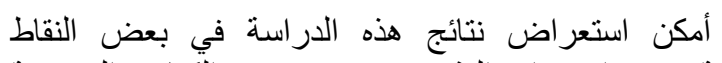
الرئيسية وهي:استخدام التشعيع في تحسين التئن الكفاءة التسويقية للمحاصيل الزر اعية موضع الدئية الدراسئ.

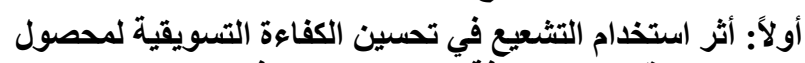
التمورقبل وبعد إضافة تكاليف التشعيع في مصني مصر.

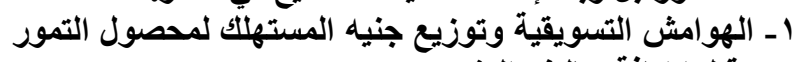
قبل إضافة تكاليف التشعيع التونيع

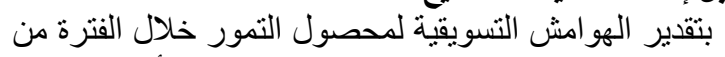

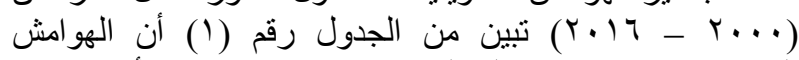

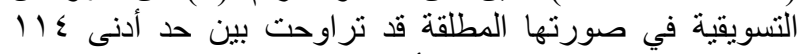

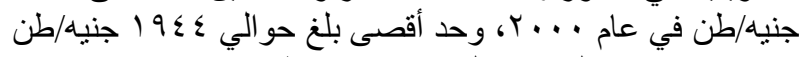

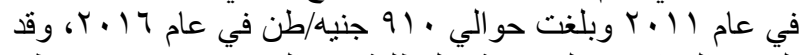

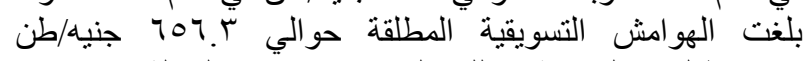

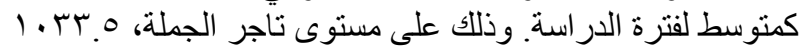
جنيه/طن على مستوى تاجر التجزئة.

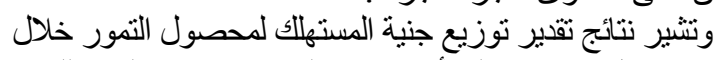

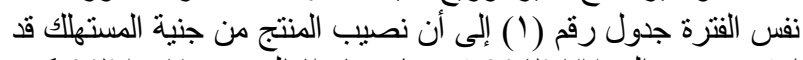

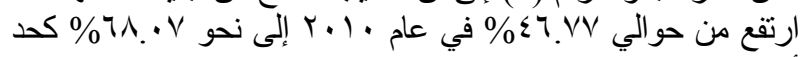

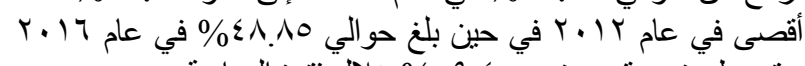

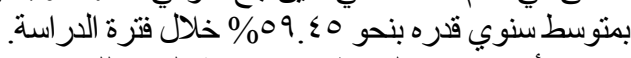

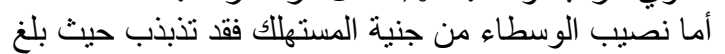

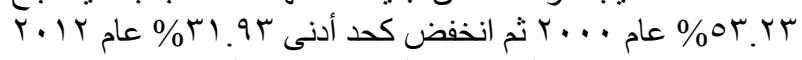

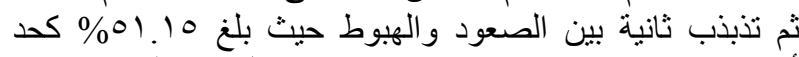

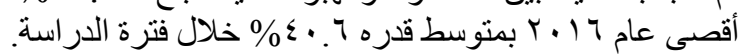

\section{مقدمة}

إمكانية استخدام المعجلات الإلكترونية في تحسين الكفاءة

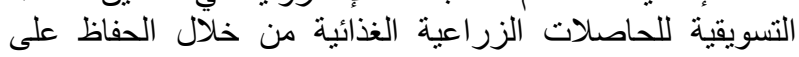

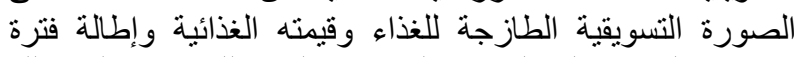

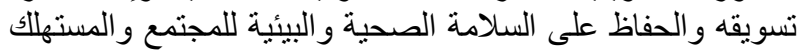

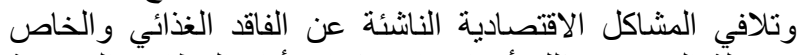

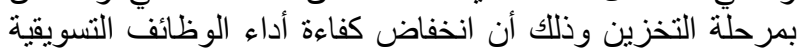

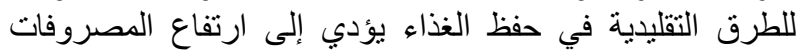

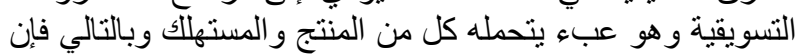

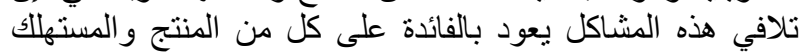

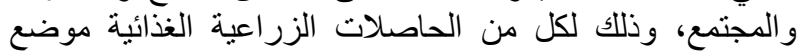

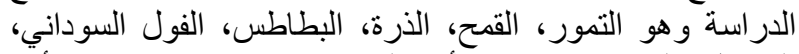

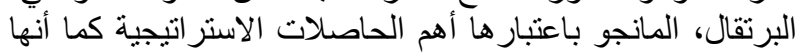

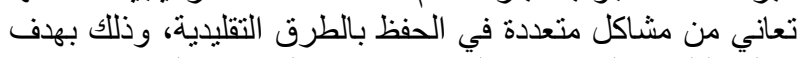

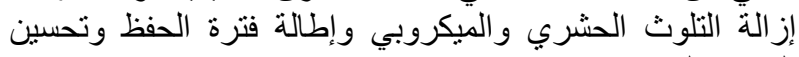
التنافس التسويقي.

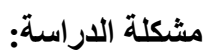

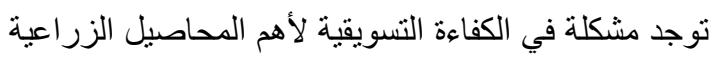

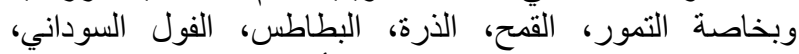

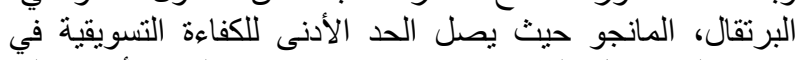

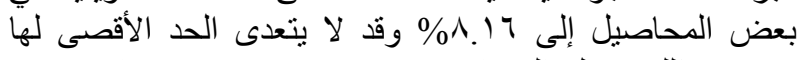

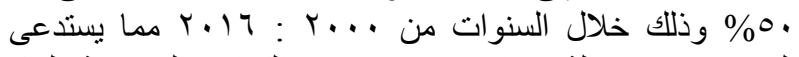

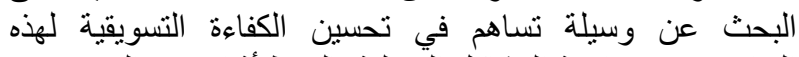
الحاصلات وبخاصة المشاكل المتعلقة بالحفظ أثناء فترة التخزين.

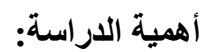

تبرز أهمية الدراسة في استخدام التشعيع في تحسين الكفاءة

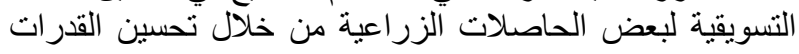
التسويقية لهذه الحاصلات وخفض الفاقد وإطالة فترة الحفة الحفظ

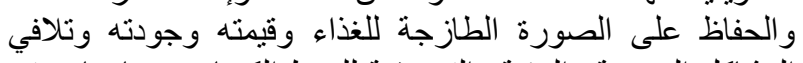

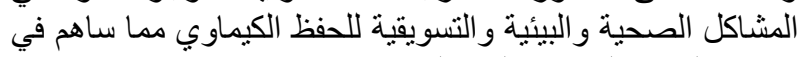

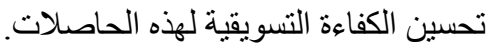

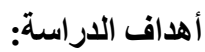

1 ـ تقدير الكفاءة التسويقية (الحد الأدنى والحد الأقصى) لمحاصيل

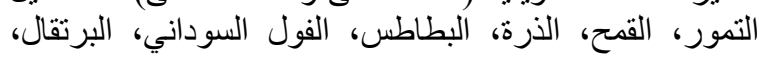

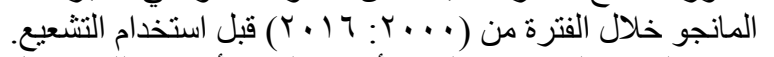
r ـ تقدير الكفاءة التسويقية (الحد الأدنى و الحد الأقصىى) للمحاصيل

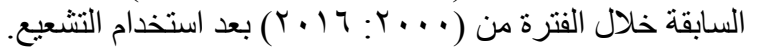




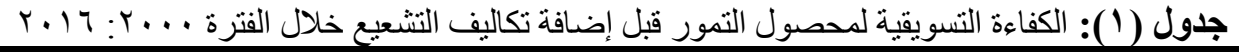

\begin{tabular}{|c|c|c|c|c|c|c|c|c|c|c|c|c|c|c|c|}
\hline \multirow{3}{*}{ التسويقية } & \multirow{3}{*}{$\begin{array}{c}\text { الوسطيبء } \\
\text { \% }\end{array}$} & \multirow{3}{*}{ نصيب تاجزة } & \multirow{3}{*}{ نصيب تاجر } & \multirow{3}{*}{$\begin{array}{l}\text { نصيب } \\
\text { \% }\end{array}$} & \multicolumn{2}{|c|}{ الانتشار السعرى } & \multicolumn{4}{|c|}{ الهوامش التسويقية } & \multirow{3}{*}{ جنيـ/طنئة } & \multirow{3}{*}{ جنيه/طن الجملة } & \multirow{3}{*}{ جنيه/طن } & \multirow{3}{*}{ 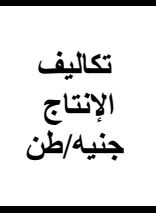 } & \multirow{3}{*}{ السنة } \\
\hline & & & & & \multirow[b]{2}{*}{ النسبى } & \multirow[b]{2}{*}{ المطلق } & \multicolumn{2}{|c|}{ هامش التجزئة } & \multicolumn{2}{|c|}{ هامش الجملة } & & & & & \\
\hline & & & & & & & النهامشب(") & المهامش (") & النسامبى(") & المهامش (") & & & & & \\
\hline ד.V.r" & זr. & $\leqslant 1.0 \leqslant$ & 11.79 & $\Sigma T . V V$ & זr.ro & 019 & $\leqslant 1.0 \leqslant$ & $\varepsilon .0$ & 11.79 & $11 \varepsilon$ & qvo & or. & $\leqslant 07$ & צT & $r \ldots$ \\
\hline$\leq \varepsilon .1$ & $\varepsilon r . r V$ & YV.qV & $1 \leq . \leq 1$ & זד. & $\varepsilon r . r V$ & $0 \ldots$ & TV.qV & שr. & $\mid \varepsilon . \varepsilon 1$ & iv. & $11 \wedge$. & 10. & $\uparrow \wedge$. & rq & $r \ldots l$ \\
\hline$\varepsilon \varepsilon, r \wedge$ & $\varepsilon r . \cdot q$ & rV.T & $1 \leq . \leqslant 1$ & ov.91 & $\varepsilon r . \cdot q$ & 099 & rV.T & rq & $1 \leq . \leqslant 1$ & $r . r$ & I & I.r. & 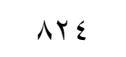 & $\varepsilon \vee \wedge$ & $r \ldots r$ \\
\hline$\leqslant \wedge .9 \leqslant$ & rv.v. & rיו.M & $10.0 \mathrm{~V}$ & שיזי & $r v . v$. & 1). & r.IT & ros & $10.0 \mathrm{~V}$ & ror & 1711 & Irצ. & $\cdots 1$ & 010 & $r \ldots r$ \\
\hline or. $\leqslant 9$ & rT.or & 17.91 & 17.74 & ג & rT.or & 010 & 17.91 & r9o & 17.74 & rq. & $1 v \leqslant 0$ & $1 \leq 0$. & 117. & TVT & $r \ldots \varepsilon$ \\
\hline or.ON & $r \leqslant . r \varepsilon$ & IV.qT & $17 . \leqslant 1$ & 70.77 & $r \varepsilon . r \varepsilon$ & $7 \wedge$. & IV.q4 & roo & 17.81 & ro & 191. & 1740 & $1, \ldots$ & Vos & r... \\
\hline 01.01 & T. Tr & $19 . \cdot v$ & 17.19 & $T \leq . V \varepsilon$ & TO.ru & YOA & $19 . v$ & «l. & 17.19 & $r \leqslant \Lambda$ & rio. & $\mid v \varepsilon$. & Irar & $\Lambda \cdot V$ & $r \ldots r$ \\
\hline$\leq 9.9 \leq$ & rV.VT & $r \cdot .90$ & 10.11 & צז. & $r v . V T$ & 101 & $r .90$ & $\leqslant 10$ & 10.11 & & ס & IAT. & $1 \leq 7 \leq$ & $\Lambda \leq q$ & $r \ldots v$ \\
\hline 1.17 & rı. $\varepsilon \varepsilon$ & o. & $10 . r 9$ & 71.07 & rA. $\varepsilon \varepsilon$ & $1.1 \varepsilon$ & r.. & 7.1 & $10 . r 9$ & $\varepsilon \cdot 7$ & אודז & $r \cdot r$. & ET & $q \leq r$ & $r \cdots \wedge$ \\
\hline$\varepsilon \varepsilon .71$ & $\varepsilon 1 . \wedge$. & TV.ro & $1 \leq .00$ & ON.Y. & ¿).^. & 101. & TV.ro & $1 . r$. & $1 \leq .00$ & 00. & TVA. & rvo. & r... & IKVT & $r \ldots q$ \\
\hline$\{0.07$ & $\varepsilon \cdot . q \mu$ & ד & $1 \leq V V$ & $09 . \cdot v$ & L. & $1 A \leq 7$ & ד.17 & 111. & $1 \leqslant V V$ & דוד & $\leqslant 01$. & שזr. & אדדז & $10 \leqslant 0$ & $r .1$. \\
\hline r..vT & $\varepsilon V . \wedge r$ & $11 . r \wedge$ & צ. דיץ & or.11 & $\sum V . \wedge r$ & Tos & $11 . r \Delta$ & $7 .$. & \&. ד & $19 \leq \varepsilon$ & orr. & EVT. & TVVT & 171. & $r .11$ \\
\hline $00 . r^{\prime}$ & r. & $1 \leq . q r$ & $I V . r$ & $\pi u_{.} \cdot v$ & r. & $\mid \wedge \Lambda \varepsilon$ & $1 \leq .9 r$ & $\wedge \Lambda$. & $I V \cdot r$ & $1 \ldots \varepsilon$ & $09 \ldots$ & O.r. & $\{.17$ & qrug & $r \cdot I r$ \\
\hline or. \&V & ס.00 & س.9. & 17.71 & 77.00 & ס.00 & ro17 & س.9. & IrV. & 17.71 & $1 r \leq 7$ & vo.. & שזיד. & $\varepsilon q \wedge \varepsilon$ & rAq) & $r .1 r$ \\
\hline$\leqslant \wedge .91$ & $\leqslant 1.71$ & $r 7.9$ & 10.09 & on.rt & $\leqslant 1.71$ & סq⿴囗十 & $r 7.99$ & riro & 10.09 & IrV. & $\lambda \mid \leq 0$ & $T \cdot r$. & $\varepsilon \vee 0$. & rro. & $r \cdot 1 \leq$ \\
\hline$\leqslant 7.0 \mathrm{~V}$ & $\leq 7 . \vee \leq$ & $r \leq . r_{0}$ & $1 r \leq 9$ & דז.ro & $\leq 7 . \vee \leq$ & $\varepsilon \cdot \wedge$. & $r \leqslant . r_{0}$ & rqq. & $1 r \leq 9$ & 1.9. & AVr. & ov $\varepsilon$. & $\leqslant 70$. & roor & $r .10$ \\
\hline$\varepsilon r .79$ & 01.10 & ¿l. & q.VV & $\sum \wedge . \wedge 0$ & 01.10 & $\sum V 70$ & $\varepsilon 1 .{ }^{\prime} \wedge$ & r & q.VV & 91. & 9410 & $0 \leq 7$. & $\leqslant 00$. & rדqV & $r .17$ \\
\hline VIr.Ao & $79 \cdot .41$ & $\leqslant 10 . \leqslant r$ & rvr.ql & $1 \cdot 1 \cdot .79$ & $99 \cdot . r 1$ & rAVYY & $\varepsilon 10 . \leqslant r$ & 18079 & rvr.qI & $1110 \mathrm{~V}$ & TaYrE & 01700 & $r \cdot \wedge \wedge . v$ & rod & الإجمالي \\
\hline$\leq \leqslant .9$ & $\varepsilon \cdot .7$ & $r \leqslant . \leqslant \varepsilon$ & 18.1 & $09 . \leqslant 0$ & $\varepsilon \cdot .71$ & 1919.89 & $r \leq . \leq \varepsilon$ & D.r. & 19.1 & 907.4 & $\varepsilon \cdot V r$ & $r \cdot r \wedge$ & $r \leqslant 1$ & 10rr.or & المتوسط \\
\hline
\end{tabular}

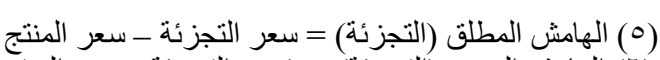

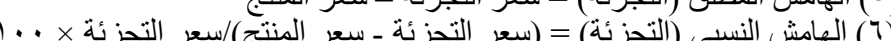

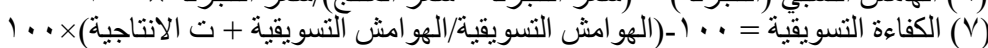

المصدر: الإدارة المركزية للاقتصاد الزر اعي ـ النشرة الاقتصادية ـ البيانات جمعت وحسبت.

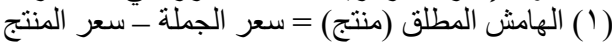

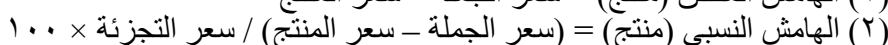

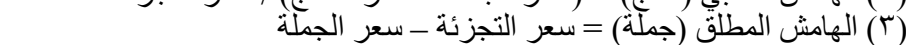

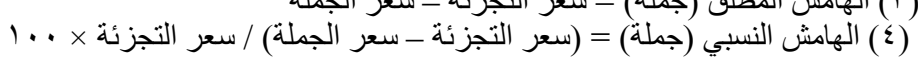




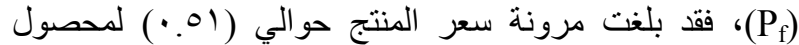

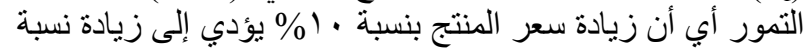

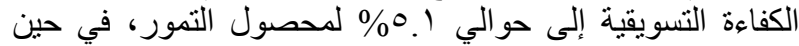

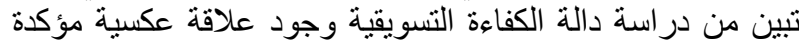

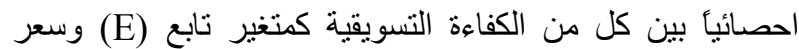

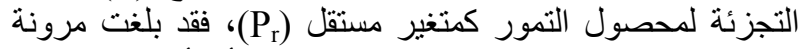

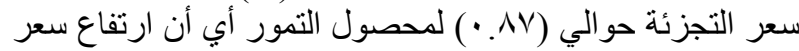

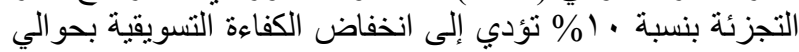

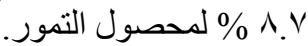

كما تبين من دراسة دالة الكفاءة التسويقية وجود علاقة طردية بين كل من الكفاءة التسويقية كمتغير تابع وتكلفة إنتاج الطن

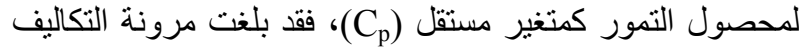
حوالي (VV. • · ) لمحصول التمور.

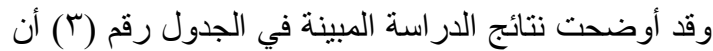

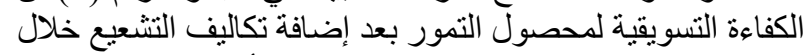

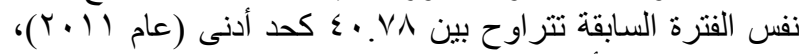

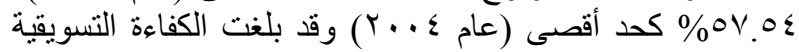

عام 17 . 17 .

كما توضح دالة الكفاءة التسويقية لمحصول التمور بعد بعد

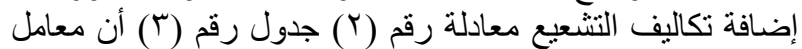

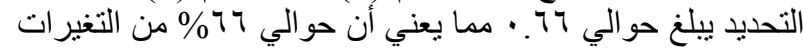

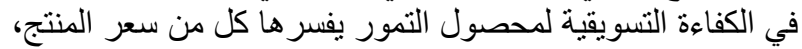

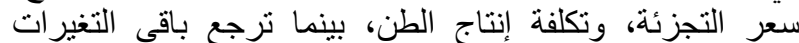
لعو امل أخرى لم تشملها دالة الكفاءة التسويقية.

حيث تبين من دراسة الكفاءة التسويقية وجود علاقة طردية

مؤكدة احصائياً بين كل من الكفاءة التسويقية كمتغير تابع (E)

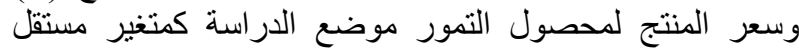

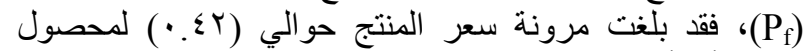

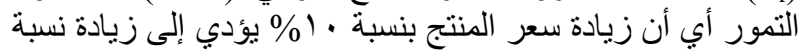

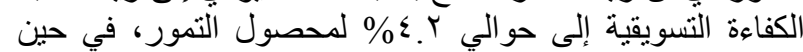

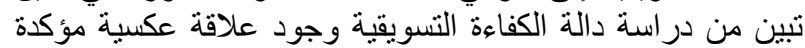

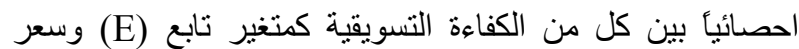

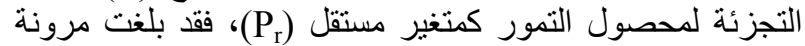

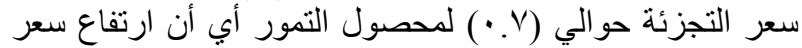

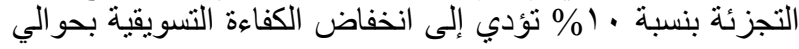

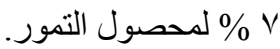

كما تبين من دراسة دالة الكفاءة التسويقية وجود علاقة

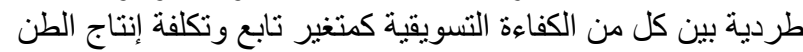

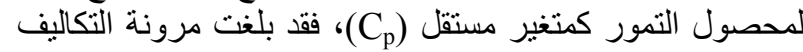
حوالي (^^ץ • ) لمحصول التمور.

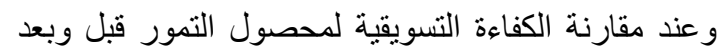

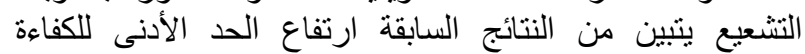

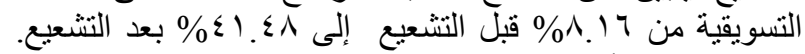

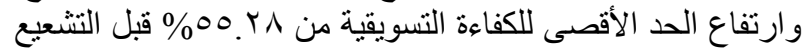

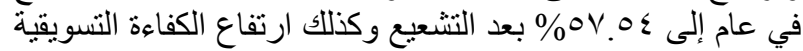

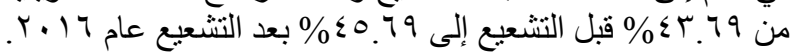

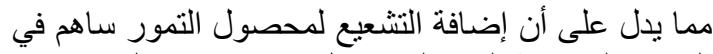

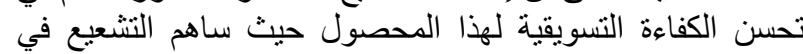

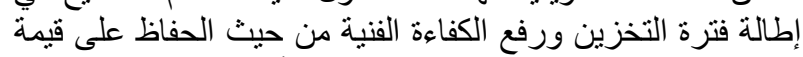

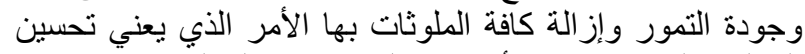
التكاليف التسويقية بنسبة أكبر من التحسين في التكاليف الاتنتاجية. r- الهوامش التسويقية وتوزيع جنيه المستهلت لمحصول التمور بعد إضافة تكاليف التثعيع:

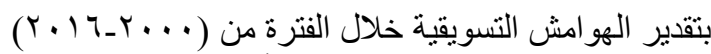

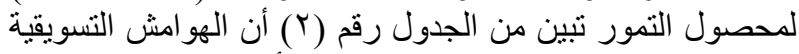

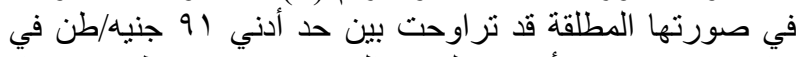

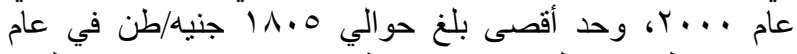

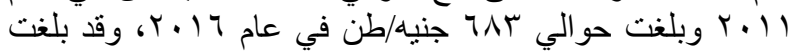

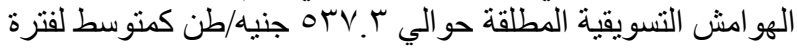

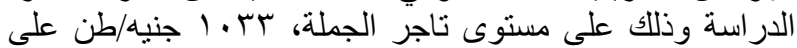

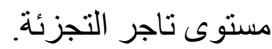

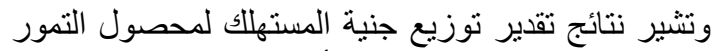

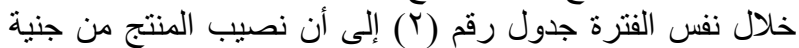

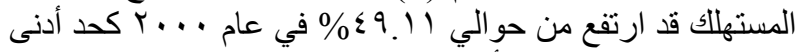

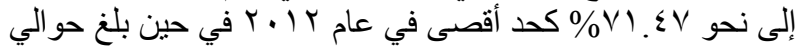

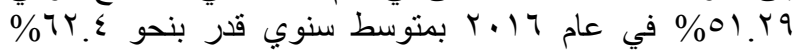

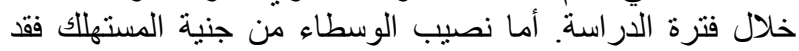

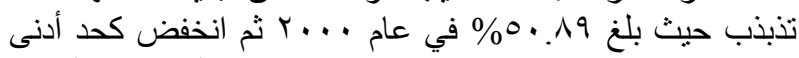

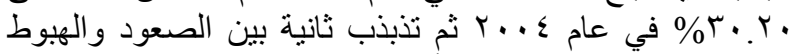

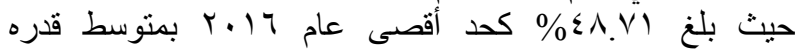

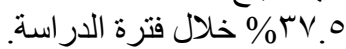

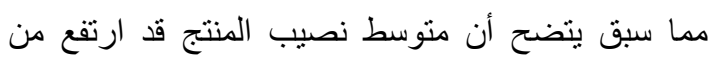

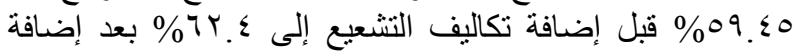

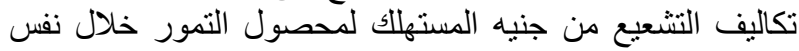

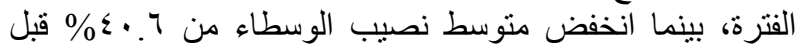

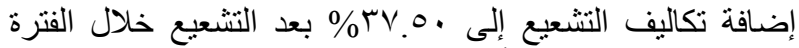

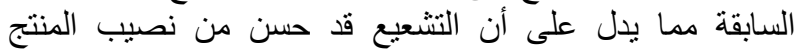

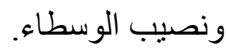

بـ العو امل المؤثرة على الكفاءة التسويقية لمحصول التمور قبل وبعد إضافة تكاليف التشعيع:

أوضحت النتائج في جدول رقم ( (1) أن الكفاءة التسويقية

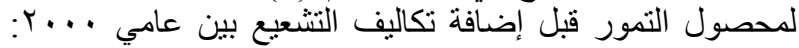

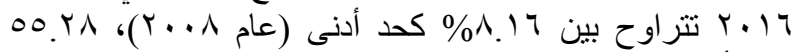

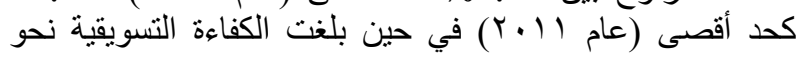

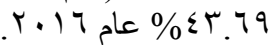

وللتعرف على طبيعة العلاقة بين الكفاءة التسويقية و العو امل

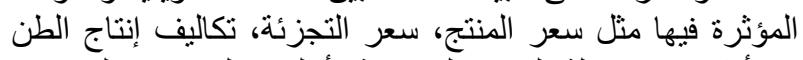

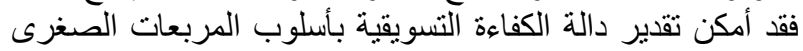

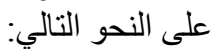

$$
\mathrm{E}=\mathrm{F}\left(\mathrm{X}_{1}, \mathrm{X}_{3}, \mathrm{C}_{\mathrm{p}}\right)
$$

تمثل دالة الكفاءة التسويقية في هذه الحالة أن الكفاءة

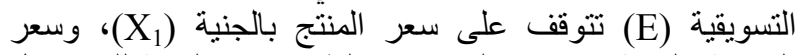

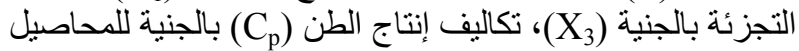

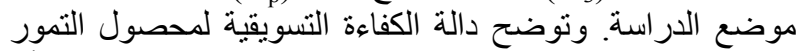

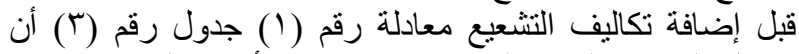

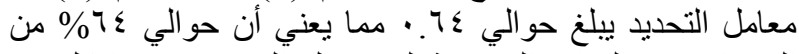

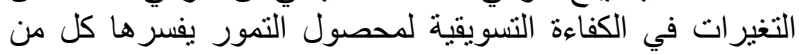

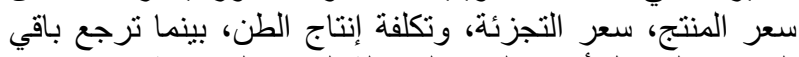
التغير ات لعو امل أخرى لم تثملها دالة الكفاءة التسويقية.

حيث تبين من در اسة الكفاءة التسويقية وجود علاقة طردية

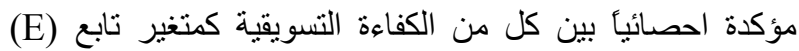

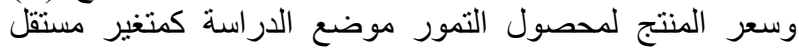




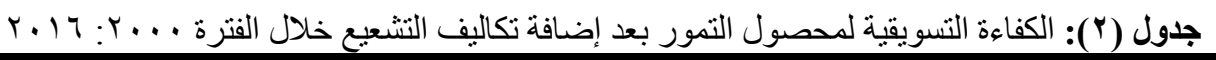

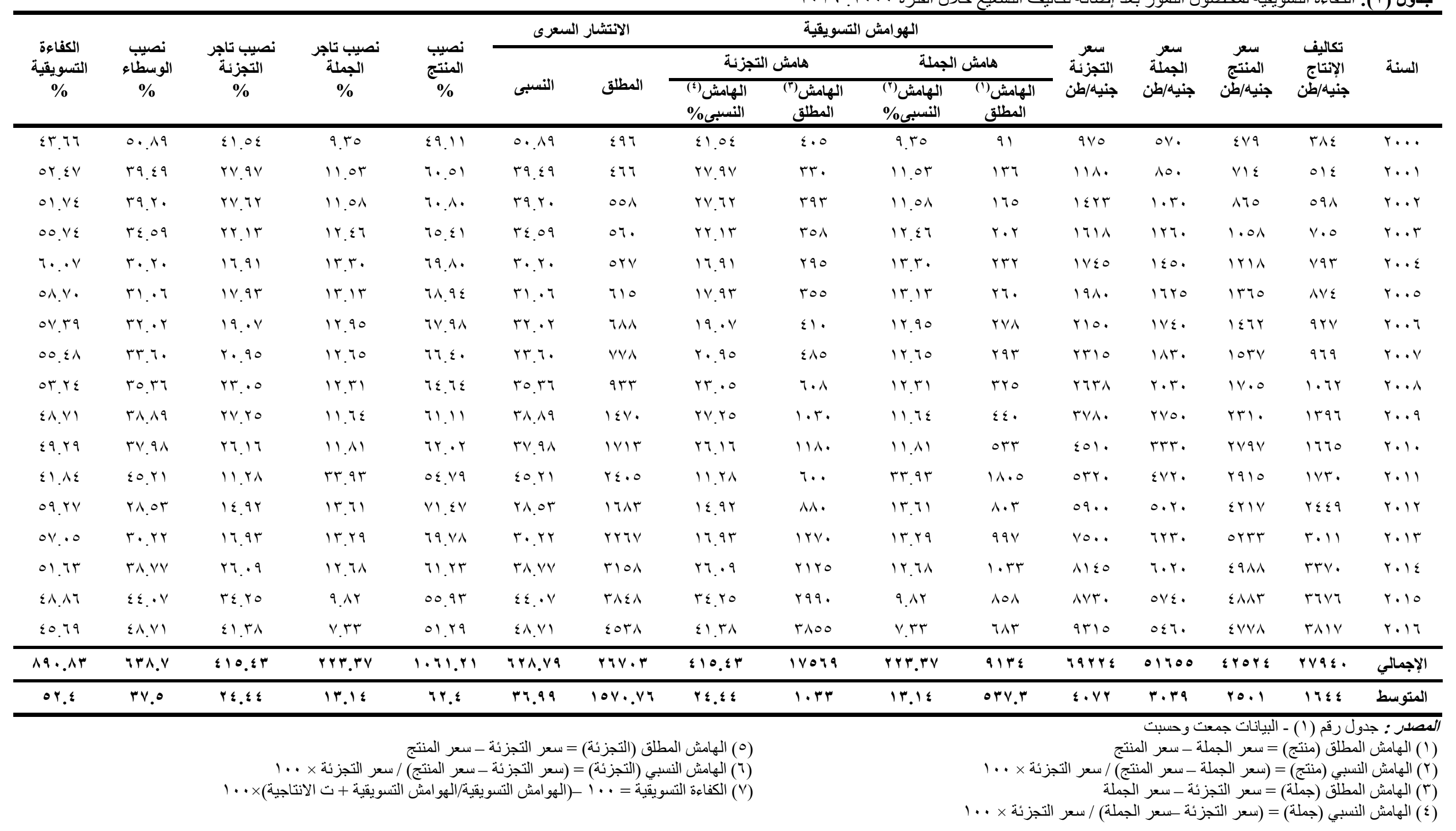




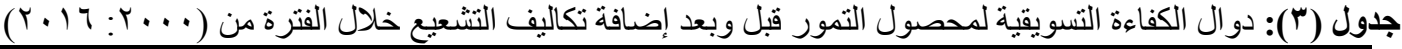

\begin{tabular}{|c|c|c|c|}
\hline $\mathbf{R}^{-2}$ & $\mathbf{F}$ & المعادلة & الت التغير \\
\hline$\because 7 \varepsilon$ & $V . V^{* * *}$ & $\begin{aligned} \mathrm{E}= & 46.93+0.011 \mathrm{P}_{\mathrm{f}}-0.013 \mathrm{P}_{\mathrm{r}}+0.017 \mathrm{C}_{\mathrm{p}} \rightarrow(1) \\
& (24.74)^{* * * *}(3.76)^{* * *}(-4.09)^{* * *}(2.45)^{* * *}\end{aligned}$ & قاكبل التشعيع التشويقية \\
\hline 0.66 & $8.437^{* * *}$ & $\begin{aligned} \mathrm{E}= & 53.68+0.009 \mathrm{P}_{\mathrm{f}}-0.009 \mathrm{P}_{\mathrm{r}}+0.0097 \mathrm{C}_{\mathrm{p}} \rightarrow(2) \\
& (28.16)^{* * *}(3.30)^{* * *}(-4.79)^{* * *}(2.40)^{* * *}\end{aligned}$ & الكفاءة التسويقية \\
\hline
\end{tabular}

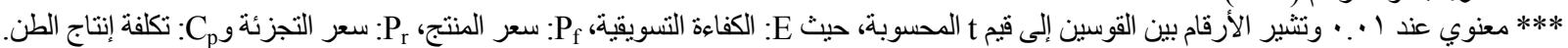

تكاليف التشعيع من جنيه المستهلك لمحصول القمح خلال الفترة

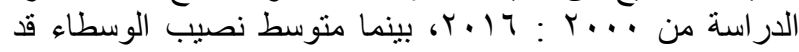

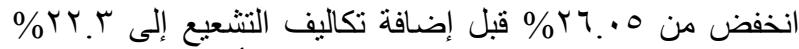

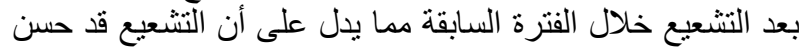
من نصيب المنتج وحد من نصيب الوسطرة الوساء.

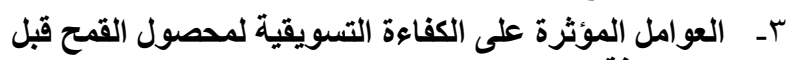
بعد إضافة تكاليف التثعيع:

أوضحت النتائج في جدول رفم (†) أن أن الكفاءة التسويقية

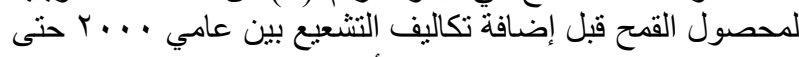

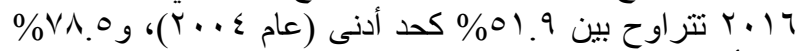

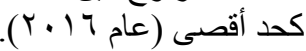
وتوضح دالة الكفاءة التسويقية لمحصول القمح معادلة رقم

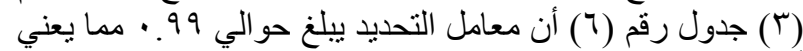

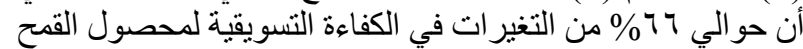

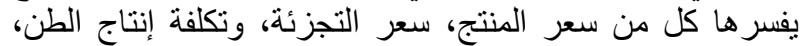
بينما ترجع باقي التغيرات لعوات امل أخرى لم تشملها دالة الكفاءة

حيث تبين من در اسة الكفاءة التسويقية وجود علاقة طردية التسويقية.

مؤكدة احصائياً بين كل من الكفاءة التسويقية كمتغير نابع (E)

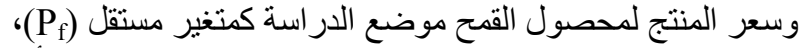

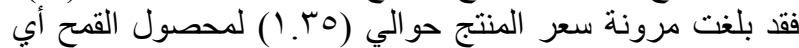

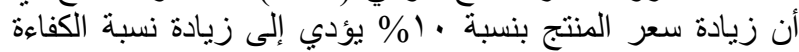

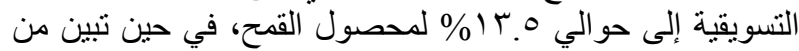

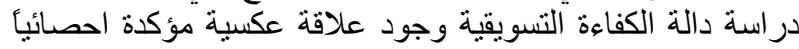

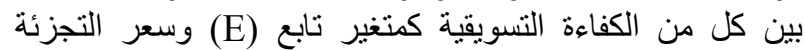

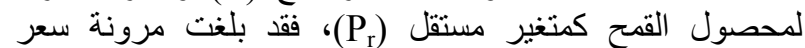

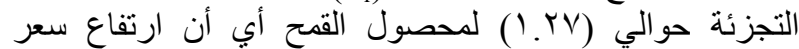

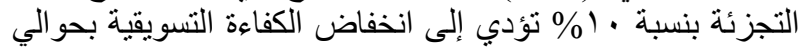

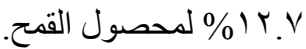

كما تبين من دراسة دالة الكفاءة التسويقية وجود علاعة علاقة طردية بين كل من الكفاءة التسويقية كمتغير تابع وتكلفة إنتاج الطن

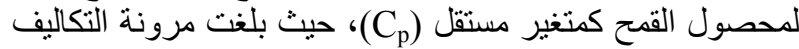
حوالي (rا . • ) لمحصول الفئرير القمح. أوضحت النتائج في الجدول رقات الفم (0) أن الكفاءة التسويقية

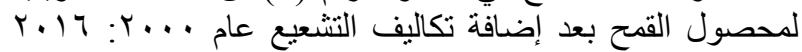

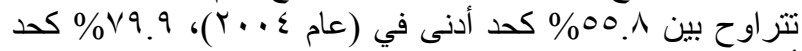

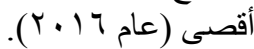

كما توضح دالة الكفاءة التسويقية لمحصول القمح بعد إضافة

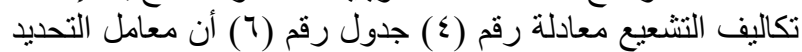
يبلغ حوالي 77.

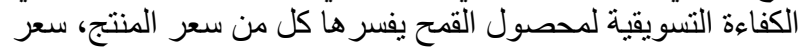

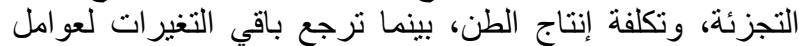
أخرى لم تشملها دالة الكفاءة التسويقية.
ثانياً: أثر استخدام التشعيع في تحسين الكفاعة التسويقية لمحصول القمحقبل وبعد إضافة تكاليف التشعيع في مصر. ا - الهوامش التسويقية وتوزيع الجنيه للمستهلك لمحصول القمح قبل إضافة تكاليف التثعيع التئيع

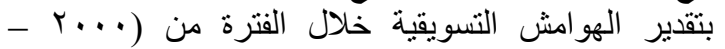

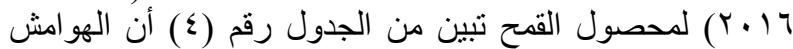

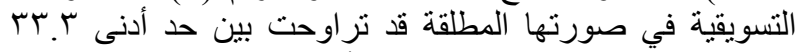

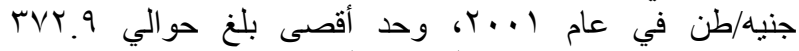

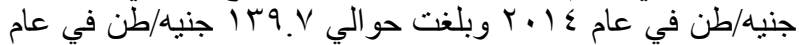

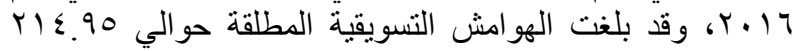

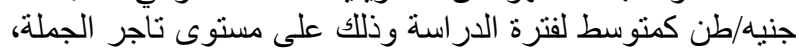

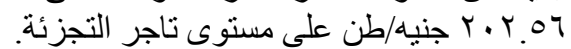

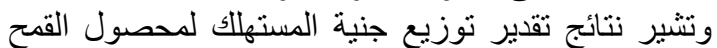

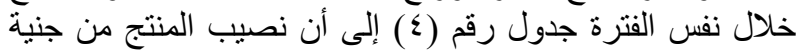

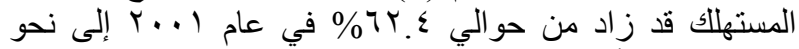

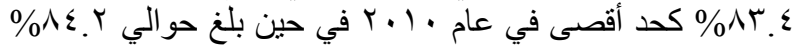

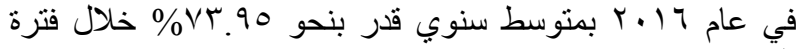

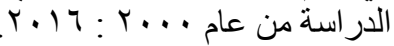

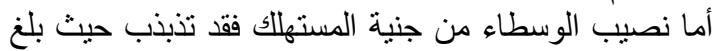

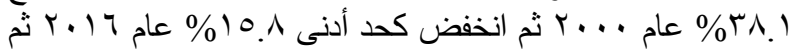

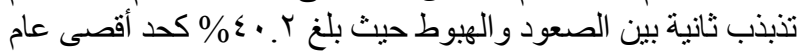

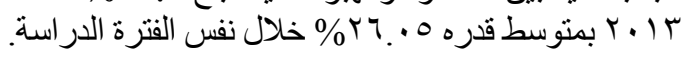

r - الهوامش التسويقية وتوزيع الجنيه للمستهلك لمحصول

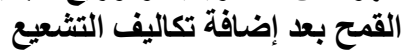

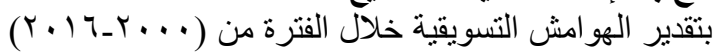

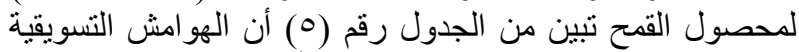

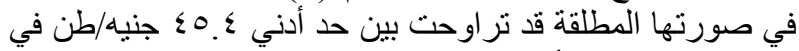

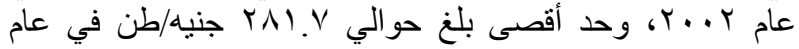

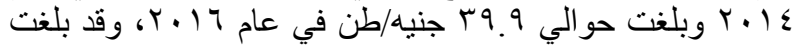

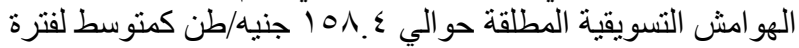

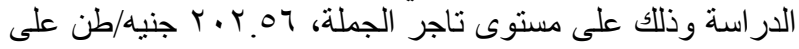
مستوى ناجر التجزئة.

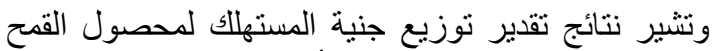

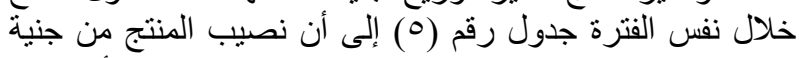

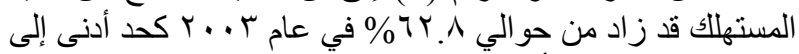

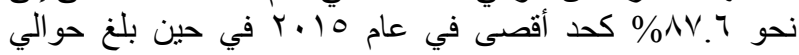

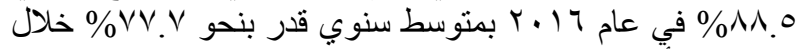

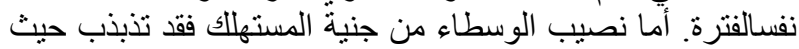

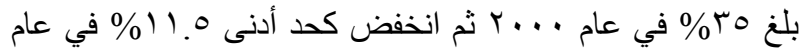

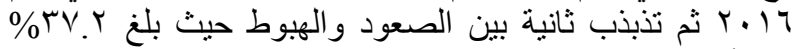

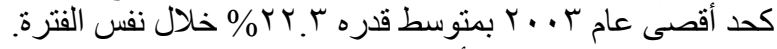

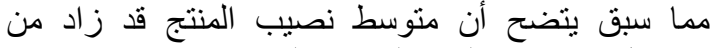

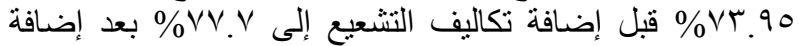




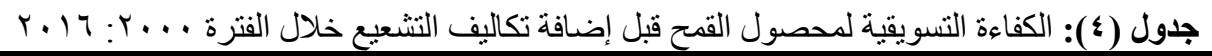

\begin{tabular}{|c|c|c|c|c|c|c|c|c|c|c|c|c|c|c|c|}
\hline \multirow{3}{*}{$\begin{array}{c}\text { التسويقية } \\
\text { \% }\end{array}$} & \multirow{3}{*}{$\begin{array}{c}\text { الوسطيب } \\
\text { \% }\end{array}$} & \multirow{3}{*}{ 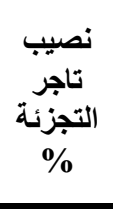 } & \multirow{3}{*}{ 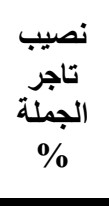 } & \multirow{3}{*}{$\begin{array}{l}\text { نصيب } \\
\text { المنتج }\end{array}$} & \multicolumn{2}{|c|}{ الانتشار السعرى } & \multicolumn{4}{|c|}{ الهوامش التسويقية } & \multirow{3}{*}{ جنيزة التجزئة } & \multirow{3}{*}{ جنيه/طن } & \multirow{3}{*}{ سنيه/طن المنتج } & \multirow{3}{*}{ جناليف الإنتاج } & \multirow{3}{*}{ السنوات } \\
\hline & & & & & \multirow{2}{*}{ النسبى } & \multirow{2}{*}{ المطلق } & \multicolumn{2}{|c|}{ هامش التجزئة } & \multicolumn{2}{|c|}{ هامش الجملة } & & & & & \\
\hline & & & & & & & الهامش(4)(النسبى\% & الهامشق(") & 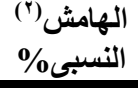 & المطلق (المش (') & & & & & \\
\hline ov. & rA. & $r 9.9$ & $\Lambda . r$ & 71.9 & rA. & $\varepsilon r v . r$ & $r 9.9$ & rmo.r & $\Lambda . r$ & qr. & IIrT & VAV & 790 & $07 V$ & r... \\
\hline ov. O & rv. & $r \leq .7$ & r. & צ. & rv. T & EYT.r & $r \leqslant . T$ & rᄉq. & $r$. & ז.Tז & Mr & VTE & $v \cdot 1$ & or. & $r \ldots l$ \\
\hline 07.1 & rᄉ. ${ }^{\prime}$ & 1.7 & $v_{.} \cdot$ & $71 . \varepsilon$ & rᄉ. $T$ & $\leqslant 01$. & r. .7 & rา.. & $v_{.}$. & NI.r & 1179 & $\vee 99$ & $\mathrm{~V} \backslash \mathrm{A}$ & orv & $r \ldots r$ \\
\hline 07. & $\varepsilon \cdot . r$ & r. $\varepsilon$ & $\mid V_{.} \wedge$ & 09.1 & $\varepsilon \cdot, r$ & 011. & Tr.s & rAะ.r & IV.A & YYT.V & $|r V|$ & $9 \wedge \mathrm{V}$ & V. & $7 \leq 9$ & $r \ldots r$ \\
\hline 01.9 & $r 9.9$ & r.. & IT.V & $7 \cdot .1$ & $r 9.9$ & . & r.. & $\varepsilon \Psi \varepsilon . \vee$ & $1 T . V$ & rYA.r & 1774 & IrTA & $1 \ldots$ & V17 & $r \ldots \varepsilon$ \\
\hline 79.8 & $r r_{.}$ & $11 . \mathrm{V}$ & $1 \cdot r$ & V^. & $r r_{.}$ & MII. & $11 . \mathrm{V}$ & $171 . V$ & $1 \cdot r$ & $1 \leq V . r$ & $1 \leq r 4$ & ITTV & Mr. & VYT & $r \ldots o$ \\
\hline 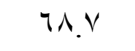 & $r \leqslant . r$ & Ir. & 11.1 & Vo.V & $r \leqslant . r$ & א.ז.r & IT & 19V. & 11.1 & $170 . r$ & $1 \leqslant 19$ & IrqY & $\| r V$ & Vq & $r \ldots r$ \\
\hline 77. & ro. $Y$ & Ir.1 & $1 T . V$ & $V \leqslant . r$ & ro. & $\varepsilon r r_{0} 0$ & Ir.1 & $191 . r$ & IT.V & TrE. & $17 \leqslant r$ & $1 \leq \leqslant \leq$ & IrT. & 119 & $r \ldots v$ \\
\hline$T V . \varepsilon$ & $r \leq .1$ & 9.9 & $1 \leq . r$ & vo. 9 & $r \leq .1$ & $\leqslant 10.0$ & 9.9 & $1 v \cdot .1$ & $1 \leq . r$ & $r \leq 0 . \varepsilon$ & $|V Y|$ & 1001 & $1 \pi \cdot 7$ & 109 & $r \ldots \wedge$ \\
\hline $71 . \wedge$ & r..$V$ & $v .9$ & $1 \leq . \wedge$ & $V V . r$ & rY.V & $\varepsilon \cdot 1.0$ & $\vee .9$ & $1 \leq 1.9$ & $1 \leq . \wedge$ & YYT.V & 11.1 & 1709 & IrqT & $9 \ldots$ & $r \ldots q$ \\
\hline$v 1.1$ & r). $\varepsilon$ & ๆ. & $10 . r$ & $\vee \wedge .7$ & Y). & $\varepsilon .1 .0$ & 7. & 111.7 & $10 . r$ & rAv.q & $1 \wedge \Lambda$. & 1V74 & $1 \leqslant \vee \wedge$ & $9 \wedge \wedge$ & $r \cdot 1$. \\
\hline$V Y . \wedge$ & $r \cdot . l$ & $\varepsilon . \varepsilon$ & 10.1 & $\vee 9.9$ & $r \cdot .1$ & ए৭ะ.० & $\varepsilon . \varepsilon$ & 10. $\varepsilon$ & 10.1 & $r .9 .1$ & 1909 & $1 \wedge V \varepsilon$ & 1070 & 1.00 & $r .11$ \\
\hline vo.A & 19. & r.A & $17 . r$ & 1). & 19. & rNv.0 & r.A & $O V . r$ & $17 . r$ & s. & $r \cdot r q$ & 1911 & 1701 & $M T$ & $r \cdot 1 r$ \\
\hline$v 7.9$ & 11. & $1 . \varepsilon$ & 17.7 & $\Delta r$. & 11. & $r \Lambda \cdot . \tau$ & $1 . \varepsilon$ & $r \wedge . q$ & 17.7 & $r 01.7$ & 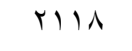 & $r \cdot 19$ & IVTV & ITIE & $r .1 r$ \\
\hline vo. 9 & 11.1 & r. $r$ & 17.7 & NI. & 11.1 & $\varepsilon r, . r$ & r.r & $\varepsilon \wedge . \varepsilon$ & 17.7 & rVY.q & $r r \leq 0$ & Y) $9 V$ & INYE & ITro & $r \cdot 1 \leq$ \\
\hline VV. $T$ & 17.7 & $\Lambda . r$ & $\wedge . \varepsilon$ & sז. & 17.7 & rvq.r & $\Lambda . r$ & IAv.r & $\Lambda . \varepsilon$ & 194. & rYAq & $r) \cdot r$ & 191. & 1411 & $r .10$ \\
\hline$\vee \wedge .0$ & 10.1 & 9.9 & 0.9 & $\Lambda \leq . Y$ & 10.1 & rVT.o & 9.9 & 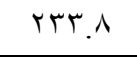 & 0.9 & $1+9.8$ & rTV. & rimt & 1997 & $1+47$ & $r .17$ \\
\hline $11 \leqslant v . V$ & $\varepsilon \leqslant$ \&.q & $r r \varepsilon . r$ & $r \cdot \Lambda . V$ & Irov. & $\leqslant \leqslant$ ५.q & VITV.T & $r r \varepsilon . r$ & $r \leqslant \varepsilon r . q$ & $r \cdot \Lambda . v$ & Mros.1 & rarrv & roßar & rrr.. & 10791 & الإجمالي \\
\hline TV.0 & $r q . .0$ & Ir.VA & IY.rA & $v r .90$ & $r 4 . .0$ & $\$ 19 . \wedge 7$ & Ir.VA & $r \cdot r .04$ & IY.rA & $r_{1} \leq .90$ & IVYO.V & lorr & $\mid r .0 . \wedge \Lambda$ & $q r r$ & المتوسط \\
\hline
\end{tabular}




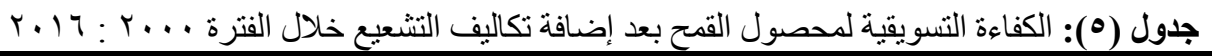

\begin{tabular}{|c|c|c|c|c|c|c|c|c|c|c|c|c|c|c|c|}
\hline \multirow{3}{*}{$\begin{array}{c}\text { التسويقية } \\
\text { \% }\end{array}$} & \multirow{3}{*}{$\begin{array}{c}\text { الوسطاء } \\
\text { \% }\end{array}$} & \multirow{3}{*}{ نصيب تاجزة } & \multirow{3}{*}{ نصيب تاجز } & \multirow{3}{*}{ نصيب المنتج } & \multicolumn{2}{|c|}{ الانتشار السعرى } & \multicolumn{4}{|c|}{ الهوامش التسويقية } & \multirow{3}{*}{ جنيه/طن التجنة } & \multirow{3}{*}{ جنيه/طن } & \multirow{3}{*}{ جنيه/طن } & \multirow{3}{*}{ تكاليف } & \multirow{3}{*}{ السنوات } \\
\hline & & & & & \multirow[b]{2}{*}{ النسبى الن } & \multirow[b]{2}{*}{ المطلق } & \multicolumn{2}{|c|}{ هامش التجزئة } & \multicolumn{2}{|c|}{ هامش الجملة } & & & & & \\
\hline & & & & & & & النهامشب(") & المامشق(") المطق & 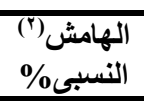 & المامشلق(') & & & & & \\
\hline ד. זד & ro." & rq. 9 & 0.1 & 10. & ro." & rqY. & rq.9 & r r. & 0.1 & OV.r & $11 K r$ & VAV & $\checkmark Y q$ & TAV & r... \\
\hline $7 \leq .1$ & $r \leqslant .0$ & $r \leqslant .7$ & $\cdot r_{-}$ & 10.0 & $r \leqslant .0$ & $r \wedge v . r$ & $r \leqslant .7$ & rیq. & $\cdot r_{-}$ & $1 . v_{.}$ & سKM & $V T \leq$ & דואי & 79. & $r \ldots l$ \\
\hline Tr.V & ro.o & M. & r.q & $7 \leqslant .0$ & ro. 0 & $\leqslant 10.1$ & T1.7 & r.9. & r. 9 & $\leqslant 0 . \leqslant$ & 1179 & $\vee १ ৭$ & Vos & $79 V$ & $r \ldots r$ \\
\hline 71.9 & $r V . r$ & r. $\varepsilon$ & $1 \leq .1$ & Tr.A & rV.r & $\varepsilon \vee r$. & rr. $\varepsilon$ & $r \wedge \leq . r$ & $1 \leq . \wedge$ & $\mid \wedge \Lambda . V$ & $|K V|$ & $9 \wedge \vee$ & $\vee १ \wedge$ & $V 79$ & $r \ldots r$ \\
\hline ov.. & r. .9 & r.. & $1 \cdot . v$ & ו.דז & $r .9$ & . זוד. & r.. & $\varepsilon r \leqslant . V$ & $1 \cdot . V$ & IVA.r & אדצ & IrYA & 1.0. & דזה & $r \ldots \varepsilon$ \\
\hline$V 7.0$ & 11.1 & $11 . \mathrm{V}$ & $7 . \varepsilon$ & 11.9 & 11.1 & r... & $11 . \mathrm{V}$ & 171.8 & $7 . \varepsilon$ & $91 . r$ & $1 \leqslant r 4$ & ITYV & $11 \times 4$ & $\lambda \leq 7$ & $r \ldots o$ \\
\hline$V \leqslant .9$ & $r \cdot .0$ & r.r. & $v . r$ & $\vee 9.0$ & $r \cdot .0$ & r. т." & Ir. & 19v. & $V . r$ & 1.9. & $1 \leqslant 19$ & Irqr & l1/r & 9) & $r \ldots r$ \\
\hline VT.Y & rr. & Ir.I & 9.9 & $\vee \wedge$. & rr. & ס.ודץ & Ir.1 & $191 . r$ & 9.9 & צ.זדו & $17 \leqslant r$ & $1 \leq \varepsilon \leq$ & $|r A|$ & 949 & $r \ldots v$ \\
\hline$V \Gamma . V$ & $r \cdot r$ & 9.9 & $1 \cdot .0$ & vq. v & $r \cdot r$ & ro. r & 9.9 & $1 v \cdot .1$ & $1 \cdot .0$ & $1 \wedge \cdot .1$ & $|V Y|$ & 1001 & $\mid$ & $9 \vee 9$ & $r \ldots \wedge$ \\
\hline vo.1 & 11.1 & V. $q$ & $1 \cdot .9$ & NI. & $1 \wedge . \wedge$ & rrs.q & $v . q$ & $1 \leq 1.9$ & $1 \cdot .9$ & 19V.. & 11.1 & 1709 & $1 \leq r r$ & $1 \cdot r$. & $r \ldots q$ \\
\hline$V V . r$ & $\mid V . \varepsilon$ & 7. & $11 . \varepsilon$ & Ar.T & $\mid V . \varepsilon$ & TrV.T & 7. & ד.r. & $11 . \varepsilon$ & YIE. & $1 \wedge 1$. & IVTT & $100 r$ & $11 \cdot 1$ & $r \cdot 1$. \\
\hline$\vee \wedge . \wedge$ & 17.1 & $\varepsilon . \varepsilon$ & 11.1 & Nז.q & 17.1 & r. & $\varepsilon . \varepsilon$ & 10. $\varepsilon$ & 11.1 & $r+. q$ & 1909 & $1 \wedge v \varepsilon$ & $17 \leq r$ & 1180 & $r .11$ \\
\hline 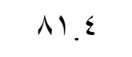 & 10. & r.A & Ir.r & 10. & 10. & r.o." & r.A & $O V . r$ & Ir.r & $r \leqslant V . \wedge$ & $r \cdot r q$ & 1911 & IVTE & Trr & $r .1 r$ \\
\hline NT.O & 17.9 & $1 . \varepsilon$ & 14.0 & ᄉт.) & $1 \% .9$ & rqז.V & $1 . \varepsilon$ & $r \wedge . q$ & $1 Y .0$ & $Y T \leq . \wedge$ & 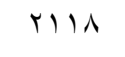 & $r \cdot \wedge q$ & INTE & IrAs & $r .1 r$ \\
\hline ᄉ). & $1 \leqslant \vee V$ & r.r & $1 r .0$ & 10.r & $1 \leqslant . V$ & Iי. & $r . r$ & $\varepsilon \wedge . \varepsilon$ & $1 r_{0} 0$ & YNI.V & $r Y \leq 0$ & Y) १V & 1910 & $1 \leq \leqslant 0$ & $r \cdot 1 \leq$ \\
\hline אז. & $1 T_{\varepsilon} \varepsilon$ & $\Lambda . r$ & $\varepsilon . Y$ & $\wedge V_{.}{ }^{7}$ & $M . \varepsilon$ & หরr.^ & $\Lambda . r$ & INV.r & $\varepsilon . r$ & 97.0 & rYAq & $r) \cdot r$ & $r \ldots o$ & $1 \leqslant r \mid$ & $r .10$ \\
\hline$\Lambda \varepsilon . \varepsilon$ & 11.0 & 9.9 & I.V & $\wedge \wedge .0$ & 11.0 & YVT.V & 9.9 & 1 & I.V & $r q .9$ & rTV. & דוrו & $r .97$ & $1 \leqslant \wedge 7$ & $r .17$ \\
\hline $1 \times 01.0$ & rvq.^ & $r r \varepsilon . r$ & $1 \leqslant V .1$ & $I r r \cdot . r$ & rvq.^ & Y.YV.A & $r r \leqslant . r$ & $r \varepsilon \varepsilon r .7$ & $1 \leqslant v . \wedge$ & หrqY.q & rqurv & YO^१Y & rrr.q & IVVYA & الإجمالي \\
\hline Vr. & YY.r & Ir.VA & 1.79 & VV.TY & rY.r & $r \Delta \leqslant .01$ & $\mid r . v \wedge$ & $r \cdot r .0 r$ & 1.79 & $101 . \varepsilon$ & IVYY & lorr & $|r v|$ & $1 \cdot \leq r$ & المتوسط \\
\hline
\end{tabular}




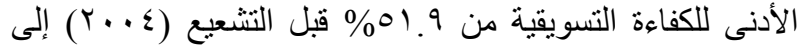

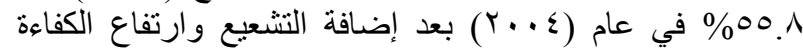

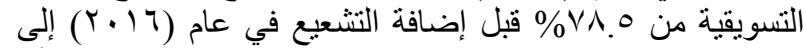

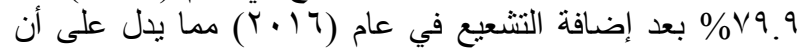

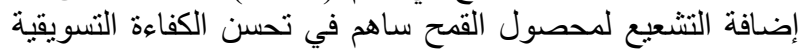

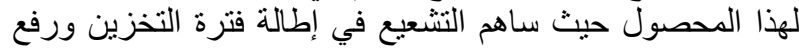

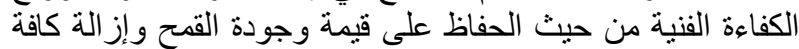

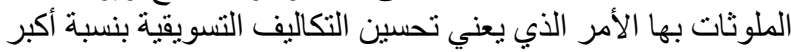
من التحسين في التكاليف الانتاجية.

كما تبين من دراسة دالة الكفاءة التسويقية وجود علائه علائة

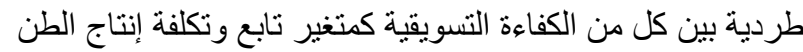

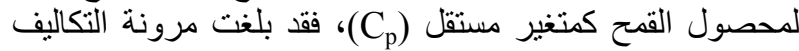
حوالي (ع ا • · ) لمحصول كئغير القمح.
حيث تبين من در اسة الكفاءة التسويقية وجود علاقة طردية مؤكدة احصائياً بين كل من الكفاءة التسويقية كمتغير تابع (E)

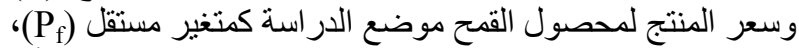

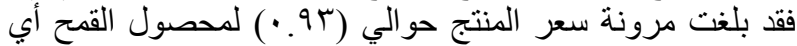

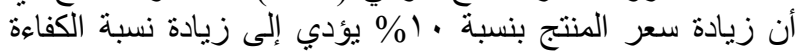

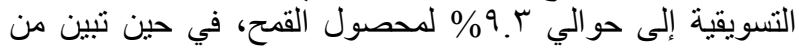

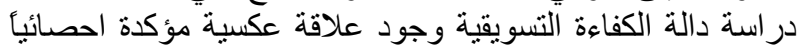

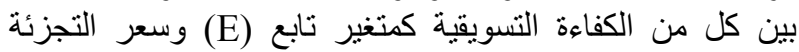

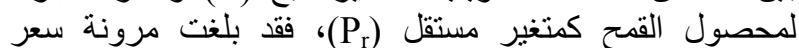

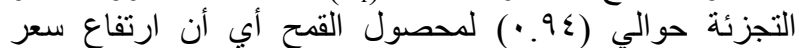

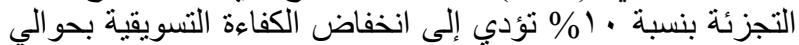

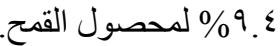
و عند مقارنة تأثير إضافة التشعيع على تحسين الكفاءة التسويقية لمحصول القمح تبين من النتائج السابقة ارتفاع إنهاع الحد

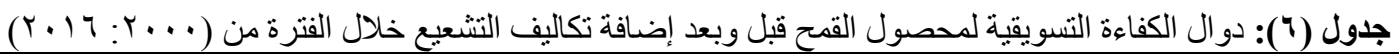

\begin{tabular}{|c|c|c|c|}
\hline $\mathbf{R}^{-2}$ & $\mathbf{F}$ & المعادلة & 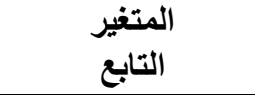 \\
\hline 0.66 & $7.7^{* * *}$ & $\begin{aligned} \mathrm{E}= & 46.93+0.011 \mathrm{P}_{\mathrm{f}}-0.013 \mathrm{P}_{\mathrm{r}}+0.017 \mathrm{C}_{\mathrm{p}} \rightarrow(3) \\
& (24.74)^{* * *}(3.76)^{* * *}(-4.09)^{* * *}(2.45)^{* * *}\end{aligned}$ & قبل تكاليف التشويقيع \\
\hline 0.99 & $8.437^{* * *}$ & $\begin{aligned} \mathrm{E}= & 53.68+0.009 \mathrm{P}_{\mathrm{f}}-0.009 \mathrm{P}_{\mathrm{r}}+0.0097 \mathrm{C}_{\mathrm{p}} \rightarrow(4) \\
& (28.16)^{* * *}(3.30)^{* * *}(-4.79)^{* * *}(2.40)^{* * *}\end{aligned}$ & بعد تكاليف التشاعة التسويقية \\
\hline
\end{tabular}

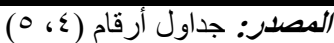

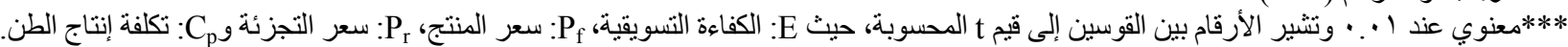

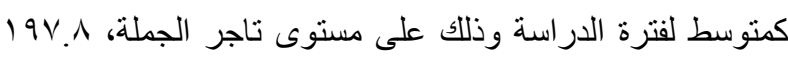
جنيا/طن على مستوى تاجر التجزئة.

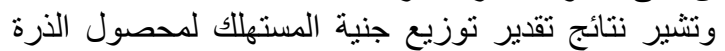
خلال نفس الفترة جدول رقم (^) تبين أن نصيب المنائ المنتج من جنية

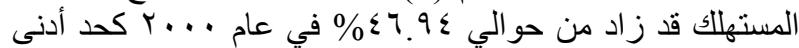

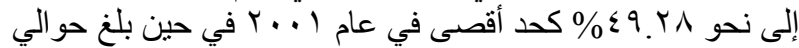

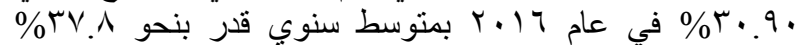

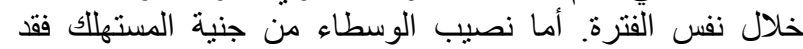

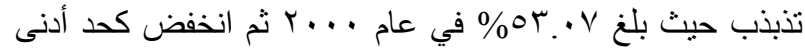

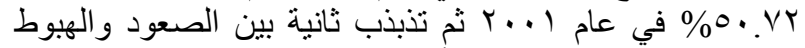

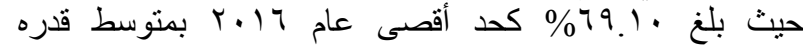
\% . IV

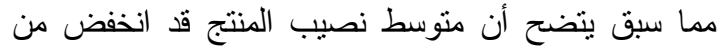

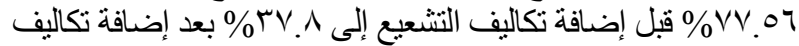

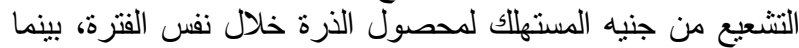

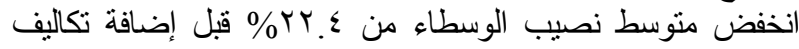

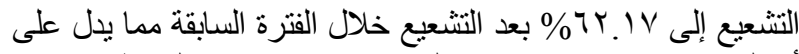

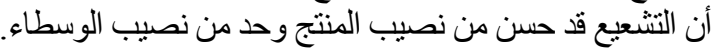

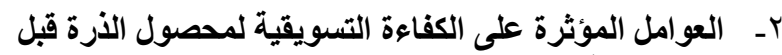

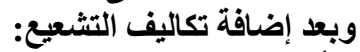

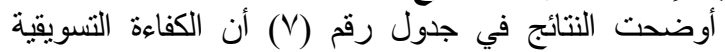

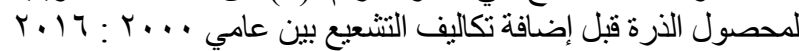

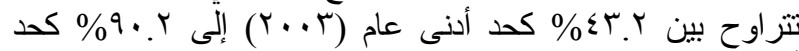

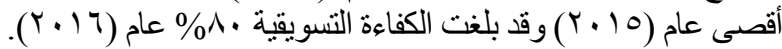

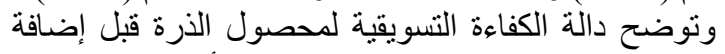
تكاليف التشعيع معادلة رقم (0) جدول رقة رقم (9) أن معامل التحديد

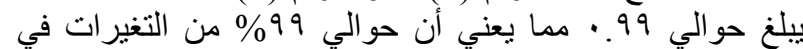

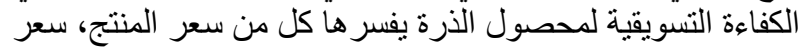

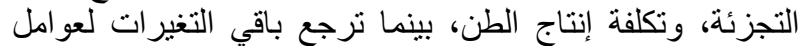
أخرى لم تشملها دالة الكفاءة التسويقية.
ثالثًا: أثر استخدام التشعيع في تحسين الكفاعة التسويقية لمحصول

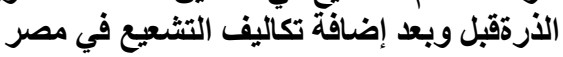

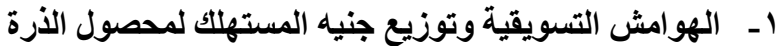
قبل إضافة تكاليف التشعيع التهويع

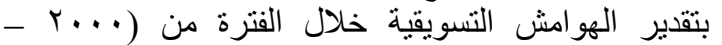

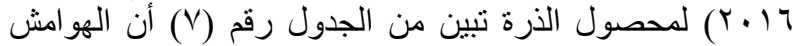

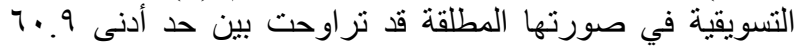

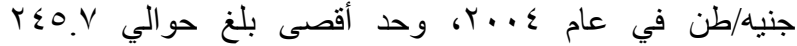

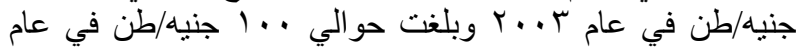

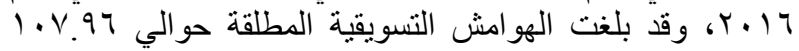
جنيه/طن كمتوسط لفترة الدراسة وذلك على الى مستوى تأجر الجملة،

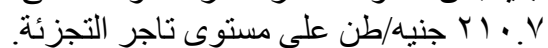

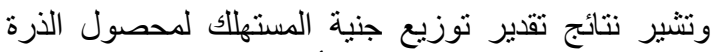

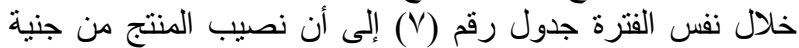

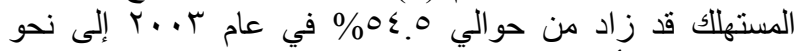

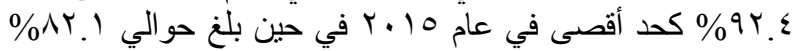

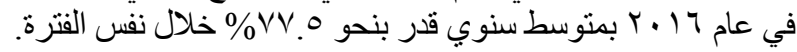

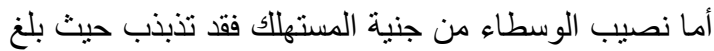

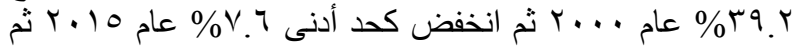

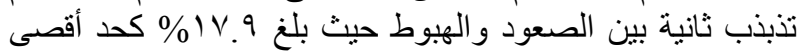

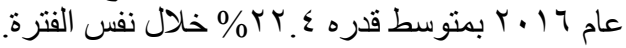

1- الهوامش التسويقية وتوزيع جنيه المستهلك لمحصول الذرة بعد إضافة تكاليف التشعيع:

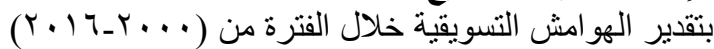
لمحصول الذرة تبين من الجدول رقم (^) أن الهو امش التش التسويقية

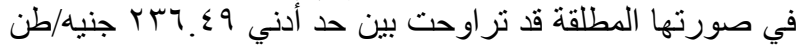

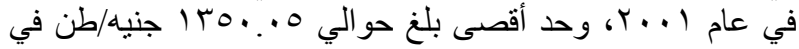

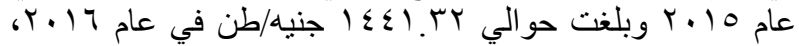

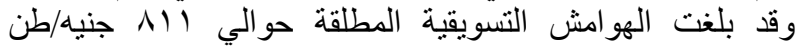




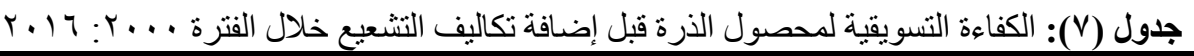

\begin{tabular}{|c|c|c|c|c|c|c|c|c|c|c|c|c|c|c|c|}
\hline \multirow{3}{*}{ التسويقية } & \multirow{3}{*}{ الوسطيب } & \multirow{3}{*}{$\begin{array}{c}\text { نتجيب } \\
\text { التجزئة } \\
\text { \% }\end{array}$} & \multirow{3}{*}{ نصيب تاجر } & \multirow{3}{*}{$\begin{array}{l}\text { المنتج } \\
\text { \% }\end{array}$} & \multicolumn{2}{|c|}{ الانتشار السعرى } & \multicolumn{4}{|c|}{ الهوامش التسويقية } & \multirow{3}{*}{ جنيه/طن التجنة } & \multirow{3}{*}{ جنيه/طن } & \multirow{3}{*}{ جنيه/طن } & \multirow{3}{*}{ جنيـاليف/طن } & \multirow{3}{*}{ السنوات } \\
\hline & & & & & \multirow[b]{2}{*}{ النسبى } & \multirow[b]{2}{*}{ المطلق } & \multicolumn{2}{|c|}{ هامش التجزئة } & \multicolumn{2}{|c|}{ هامش الجملة } & & & & & \\
\hline & & & & & & & 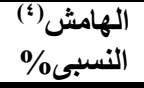 & الهامشق (") & 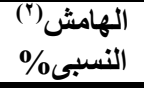 & المطلق (المشق & & & & & \\
\hline or. & rq. & rv.q & $11 . \varepsilon$ & $7 \cdot .1$ & rq. & rqז.l & rv.q & rVA.T & $11 . \varepsilon$ & $11 r .0$ & $1 \ldots$ & VYI & $7 \cdot 1$ & $\leqslant r_{0}$ & $r \ldots$ \\
\hline 01.7 & r9.0 & $r V . \varepsilon$ & Ir.1 & $7 . .0$ & r9.0 & $\varepsilon \cdots 1$ & $r V . \varepsilon$ & rVV.r & Ir.1 & Irr.A & 1.11 & VTr & זוד & $\varepsilon r V$ & $r \ldots l$ \\
\hline$\leqslant 0.1$ & $\leqslant 0$. & r.. & 14.9 & 00. & $\leq 0$. & $01 r .9$ & r. & א.4.人 & 14.9 & $1 \leq V_{.} 1$ & $11 \leqslant r$ & VVT & $7 r 9$ & $\leqslant$ ro & $r \ldots r$ \\
\hline$\varepsilon r . r$ & $\leqslant 0.0$ & r. & $19 . r$ & $0 \leqslant 0$ & $\leqslant 0.0$ & 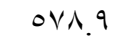 & r.. & r. & $19 . r$ & $r \leqslant 0 . V$ & ITVY & 949 & $79 \pi$ & $\leq \varepsilon 1$ & $r \ldots r$ \\
\hline r & $r$ r.I & 110.0 & $\varepsilon .0$ & $v 7.9$ & rr.l & r. & 11.0 & $r \leq q . \varepsilon$ & $\varepsilon .0$ & $7 \cdot .9$ & $1 \pi \leq 7$ & $1.9 V$ & 1.47 & orr & $r \ldots \varepsilon$ \\
\hline 70.5 & rT.o & 10.4 & $\Lambda_{.} \varepsilon$ & $V 7.0$ & rr.o & ris. 9 & $10 . r$ & $r .0 . r$ & ^. $\varepsilon$ & 114.7 & 1500 & $11 \leqslant 9$ & 1.47 & $7 . r$ & $r \ldots o$ \\
\hline $77 . Y$ & rr. & $10 . \mathrm{V}$ & $V_{.} \varepsilon$ & $v 7.9$ & $r$ r.l & $r Y \varepsilon . \varepsilon$ & $10 . \mathrm{V}$ & $r Y \cdot .1$ & $V_{.} \varepsilon$ & $1 \cdot \varepsilon . r$ & $1 \varepsilon \cdot r$ & 111 & 1.19 & דזד & $r \ldots r$ \\
\hline$v \cdot . v$ & r). $\varepsilon$ & $1 \leq .1$ & V.r & $\vee \wedge .7$ & r). & $r r \leqslant . r$ & $1 \leq .1$ & TIT.s & V.r & $11 \cdot .1$ & 1011 & $1 \pi .0$ & $119 \varepsilon$ & $\vee \wedge \varepsilon$ & $r \ldots v$ \\
\hline$v 7.7$ & 19.1 & IT. & 7. V & $1 \cdot .9$ & 19.1 & $r \cdot r . q$ & IT. $\varepsilon$ & $19 V .1$ & $7 . V$ & 1.7 .1 & $109 \pi$ & 11490 & $1 \% \wedge 9$ & 994 & $r \ldots \lambda$ \\
\hline vq.1 & IV. & $1 \cdot .9$ & 7.1 & Ar. & IV. & KAT.T & $1 \cdot .9$ & IAr.r & 7.1 & $1.1 . \varepsilon$ & 1771 & $1 \leqslant \wedge 7$ & IT人E & $1 \cdot v \varepsilon$ & $r \ldots q$ \\
\hline 1). $\varepsilon$ & 10.1 & 9.7 & 0.0 & $\lambda \leq .9$ & 10.1 & rד. & 9.7 & 177.7 & 0.0 & $97 . \mathrm{V}$ & $I V \leqslant r$ & $10 V 7$ & $1 \leq \vee 9$ & 1100 & $r \cdot 1$. \\
\hline Ar. & $1 T . \varepsilon$ & $\Lambda . r$ & 0.1 & 17.7 & $1 T . \varepsilon$ & $r \leq r . q$ & $\Lambda . r$ & 101. & 0.1 & 91.9 & INIV & 1777 & $10 V \leqslant$ & 119. & $r+11$ \\
\hline$\wedge \leq . \wedge$ & 11.1 & Y. $Y$ & $\varepsilon .7$ & $\Lambda \Lambda . r$ & 11.1 & MrY.T & V.r & 1ro.s & $\varepsilon .7$ & AV. $Y$ & 1194 & IVOV & 178. & $1 K \leq 7$ & $r \cdot I r$ \\
\hline$\wedge \uparrow . \wedge$ & $1 \cdot r$ & 7.1 & $\varepsilon . r$ & ^৭. . & $1 \cdot r$ & $r \cdot r \cdot r$ & 7.1 & 119.9 & $\varepsilon . r$ & Nr.o & $197 \mathrm{~V}$ & $1 A \leqslant V$ & $1 \times 70$ & ITr. & $r \cdot 1 r$ \\
\hline$\wedge \Lambda . \varepsilon$ & $\wedge .9$ & 0.1 & r.A & 91.1 & 1.9 & 111.9 & 0.1 & $1 \cdot \varepsilon . r$ & r.A & $V V . \wedge$ & $r \cdot \leq r$ & 1941 & 111. & $1 \pi q$. & $r \cdot 1 \leq$ \\
\hline$q \cdot . r$ & $\vee .7$ & $\varepsilon . r$ & r.o & $94 . \varepsilon$ & $v .7$ & 171.7 & $\varepsilon . r$ & $\wedge \wedge .7$ & r.o & $v r$. & YIV & $r \cdot r \wedge$ & 1900 & $1 \leqslant \wedge \varepsilon$ & $r \cdot 10$ \\
\hline$\wedge \cdot$. & 18.9 & IT. & $\varepsilon .7$ & Ar.l & 18.9 & rq1.0 & $1 T . r$ & Y91.0 & $\varepsilon .7$ & $1 \cdots$ & 1191 & $19 \ldots$ & $11 \ldots$ & $107 \varepsilon$ & 4.17 \\
\hline $1 \% \cdot \Lambda . \varepsilon$ & 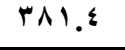 & Yos. $r$ & $1 Y V_{. \varepsilon}$ & $1 \% 1 \wedge .7$ & rᄉ . & $0 \leq 17 . r$ & YO.$Y$ & rosI.r & Irv.s & INHo.r & $r v \cdot \Lambda \cdot$ & $r r \leq q q$ & Y177ะ & IVY7 & الإجمالي \\
\hline$v 1 . \cdot 1$ & YY. & $1 \leq .90$ & $v .0$ & $v v_{.0}^{\circ}$ & YY.\& & M1^.7 & $1 \leqslant .90$ & $r \cdot . v$ & $v_{0} 0$ & $1 \cdot 8.97$ & 1094 & IrAr & IYVE & 1.10 & المتوسط \\
\hline & & جية) & سعويقية + ن ن التجئ & سعر المعر المنت. & امش التجزئ التُّا & 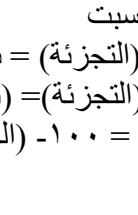 & لكامش الهاءث المطب النسب & & & |زئة ×. & الإدارة المركر & جزئة -سعر - سعر & 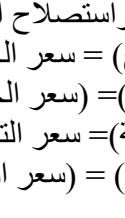 & للاقل الزب (منتة & 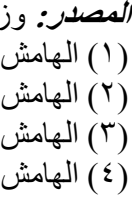 \\
\hline
\end{tabular}




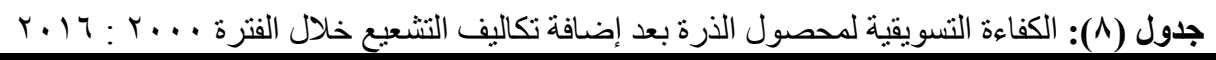

\begin{tabular}{|c|c|c|c|c|c|c|c|c|c|c|c|c|c|c|c|}
\hline \multirow{3}{*}{ التسويقية } & \multirow{3}{*}{$\begin{array}{c}\text { نصيب } \\
\text { \% }\end{array}$} & \multirow{3}{*}{ نصيب تاجز } & \multirow{3}{*}{ نصيب تاجز } & \multirow{3}{*}{ نصيب } & \multicolumn{2}{|c|}{ الانتثار السعرى } & \multicolumn{4}{|c|}{ الهوامش التسويقية } & \multirow{3}{*}{ جنيه/طن التزة } & \multirow{3}{*}{ جنيه/طن الجملة } & \multirow{3}{*}{ جنيه/طن } & \multirow{3}{*}{ تكاليف الإتتاج } & \multirow{3}{*}{ السنوات } \\
\hline & & & & & \multirow[b]{2}{*}{ النسبى } & \multirow[b]{2}{*}{ المطلق } & \multicolumn{2}{|c|}{ لـامبر التجزئة } & \multicolumn{2}{|c|}{ هامش الجملة } & & & & & \\
\hline & & & & & & & الهامش (₹) النسبى\% & المطامش") & 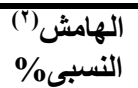 & الهامشق(") & & & & & \\
\hline$\leq 0 . V$. & or..v & rV.AT & ro.r. & $\leq 7.9 \leq$ & or. . v & or. . V & rV.AT & TVA.T. & YO. Y & ror..o & $1 \ldots$ & VYI & $\leqslant 79$ & $\varepsilon \leqslant V$ & $r \ldots$ \\
\hline$\leq 7.7 T$ & $0 . V Y$ & $r v . r v$ & סז.זץ & $\leqslant 9 . Y \wedge$ & $0 . V Y$ & $01 \Gamma . \wedge$ & $r v . r v$ & TVV.T. & סז.דז & rrד. $\leqslant q$ & 1.14 & & $\leqslant 99$ & $\leq \leqslant 9$ & $r \ldots l$ \\
\hline$\varepsilon r .+1$ & or. $9 \leq$ & rr. $q$ & $r \cdot .10$ & $\varepsilon V .+T$ & or. $9 \leq$ & $7.0 . \mathrm{Y}$ & r. 9 & . & $r \cdot .10$ & זr.ґ & $11 \leq r$ & VVT & ors & EOV & $r \ldots r$ \\
\hline rv.o. & $7 . . T Y$ & r... & $r \leqslant . \leqslant r$ & rq.r & $7 . .74$ & $V V \cdot .9$ & r... & •.ץ"זr & $r \leqslant . \leqslant r$ & $\varepsilon r V . V)$ & ITVK & 949 & 0.1 & $\leq \pi$ & $r \ldots r$ \\
\hline$\varepsilon \cdot . \wedge V$ & $7 . .11$ & 11.04 & $\leqslant 1.01$ & $r q . \wedge 9$ & $7 . .11$ & 1.9 .1 & 11.04 & $r \leq 9 . \varepsilon$. & $\leqslant 1.01$ & 009.71 & $1 \pi \leq 7$ & $1.9 \mathrm{~V}$ & orv & 009 & $r \ldots \varepsilon$ \\
\hline$\varepsilon r . V T$ & Tצ.T & 10.17 & $\leqslant V . \leqslant q$ & דז. & TY.TE & $\wedge \leqslant \wedge .7$ & 10.17 & r.o.r. & $\leqslant V . \leqslant q$ & $T \leq r . Y_{0}$ & 1500 & $11 \leq 9$ & 0.7 & אזד & $r \ldots o$ \\
\hline$\leqslant 0.01$ & or.Ar & 10.79 & $\leq 1.1 \leq$ & $\varepsilon r .1 V$ & & V৭V.r & 10.79 & $r r \cdot .1$. & $\leq 1.1 \leq$ & OVV.rT & $1 \varepsilon \cdot r$ & 111 & 7.7 & 771 & $r \ldots r$ \\
\hline$\leqslant 0 . \vee 7$ & $T \leqslant . T V$ & $1 \varepsilon .7$ & $0 . Y_{1}$ & ro.VT & $T \varepsilon . Y V$ & qvo. & $1 \varepsilon .7$ & rIT. & $0 . M_{1}$ & דצYYT & 1011 & $1 \pi .0$ & $0 \leq r$ & NTr & $r \ldots v$ \\
\hline ט. & זr.זד & $M . \leqslant r$ & 0.9. & גו.ד & זיזיד & $1 \cdots 1.7$ & $M . \leqslant r$ & $19 \vee . \wedge$. & 0.9. & $11 \cdot . \vee q$ & 1094 & 1490 & $0 \wedge \leq$ & $1 . \leqslant r$ & $r \ldots \wedge$ \\
\hline $01 . Y \leq$ & ס & $1.9 r$ & or. $\leqslant$ r & ro. 70 & ס ז. T & I.Vr.r & $1.9 r$ & 1Ar.19 & or. $\leqslant$ r & $19 \cdot .91$ & 1771 & $1 \leq \Lambda 7$ & 090 & IIK & $r \ldots q$ \\
\hline or.rv & ש.r.זי & 9.07 & or.v & rq.v. & ש.r.זד & $11 . r .1$ & 9.07 & 177.09 & or.v & 947.01 & $1 V \leqslant r$ & $10 V 7$ & $7 r q$ & MTIT & $r \cdot 1 \cdot$ \\
\hline 01.9 & To.Ar & A.r. & $0 V .01$ & $r \varepsilon .11$ & $70 . \wedge \mathrm{r}$ & 1197.4 & $\Lambda . M$ & 10.99 & $0 V .01$ & $1 . \leq 0.19$ & INIV & 1777 & TY & iro. & $r .11$ \\
\hline $01.1 \leq$ & & $v .10$ & $09.1 \mathrm{~V}$ & גז.דז & r.r. & 1 ros.9 & V.10 & 1ro.r. & $09.1 \mathrm{~V}$ & $1119.0 \mathrm{~V}$ & 1194 & IVOV & TrV & $1 \mu \cdot \Lambda$ & $r \cdot 1 r$ \\
\hline 01.19 & $T V . \leqslant Y$ & 7.9 & $\pi$ ז & M.OA & $T V . \leqslant Y$ & Irrt.r & 7.99 & 119.81 & TI. & $M \cdot T . \leqslant V$ & 1978 & $1 \wedge \leqslant V$ & $7 \leqslant 1$ & IT TQV & $r .1 r$ \\
\hline $01 . Y \leq$ & $r \Lambda \cdot r$ & 0.1 & Tr.qY & $r 1.91$ & $r \Lambda \cdot r$ & $1 r \wedge \Lambda . q$ & 0.1 & $1 . \leq .11$ & Tr.qY & IrAE.VO & $r \cdot \leq r$ & $194 \wedge$ & ror & $1 \leq 7$. & $r \cdot 1 \leq$ \\
\hline 01.99 & $7 V .9 V$ & $\varepsilon .11$ & ז.. & rr. r & $7 V .9 V$ & $1 \leq r \wedge .7$ & $\{.11$ & $1 \wedge .0 V$ & Tr.v & 1 1ro... & YIV & $r \cdot r \Lambda$ & $T \vee \wedge$ & 1001 & $r .10$ \\
\hline Or. . r & 79.1. & זr. & $70 . V V$ & $r . .9$. & 79.1. & $101 \leqslant . r$ & זr. & $V Y .9 V$ & $70 . V V$ & $1 \leq \varepsilon 1 . r Y$ & 1991 & 5119 & TVV & $17 \leqslant r$ & $r .17$ \\
\hline$\wedge 1 \cdot . \wedge \wedge$ & $1.04 . \wedge r$ & $r \leq \varepsilon . \cdot r$ & $\Lambda \mid r . \Lambda$ & $7 \leq r .19$ & $1.04 .1 \mathrm{r}$ & $171 \vee 9.7$ & $r \leq \varepsilon . \cdot r$ & PrY.00 & $\Lambda ! r . \Lambda$ & rVqY. & $I V \cdot A$ & rrvis & q৭४r & $17 \leqslant 9 \wedge$ & الإجمالي \\
\hline$\leq \vee . V$ & TY.IV & $1 \leq .40$ & $\varepsilon V . \wedge$ & $r v . \wedge$ & TY.IV & $901 . V$ & $1 \leq .40$ & $19 \vee . \wedge$ & $\leq V . \wedge$ & 111 & $109 \varepsilon$ & $1 r 90$ & O^r & $9 \vee$. & المتوسط \\
\hline & 1 & & تُ تج التسوي / سع & لَتسئة - سـة سـ & = =سعر & 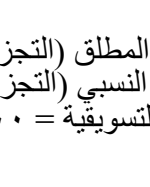 & 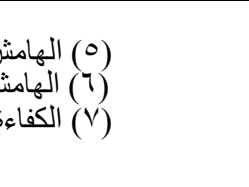 & & & כא' & 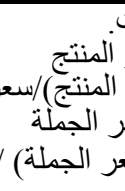 & تجزئة ـــ سـ & (س) & النسبي & (1) \\
\hline
\end{tabular}




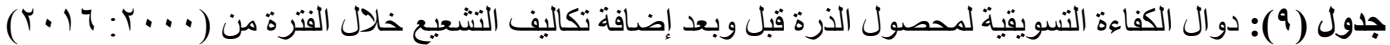

\begin{tabular}{|c|c|c|c|}
\hline $\mathbf{R}^{-2}$ & $\mathbf{F}$ & المعادلة & الت التغير \\
\hline 0.99 & $236.8^{* * *}$ & $\begin{aligned} \mathrm{E}= & 66.63+0.07 \mathrm{P}_{\mathrm{f}}-0.06 \mathrm{P}_{\mathrm{r}}+0.01 \mathrm{C}_{\mathrm{P}_{* * *} \rightarrow(5)} \\
& (9.79)^{* * *}(9.37)^{* * *}(-5.9)^{* * *}(1.88)^{* * *}\end{aligned}$ & قالكفاءة التسويقية \\
\hline 0.99 & $440.1^{* * *}$ & $\begin{aligned} \mathrm{E}= & 71.39+0.06 \mathrm{P}_{\mathrm{f}}-0.06 \mathrm{P}_{\mathrm{r}}+0.01 \mathrm{C}_{\mathrm{p}} \rightarrow(6) \\
& (15.57)^{* * *}(10.82)^{* * *}(-8.65)^{* * *}(3.34)^{* * *}\end{aligned}$ & بعد إضافة التشويقية \\
\hline
\end{tabular}

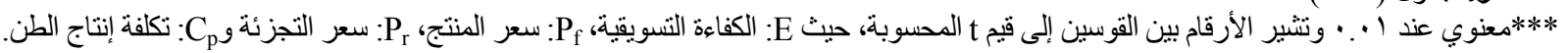

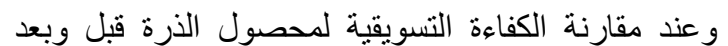

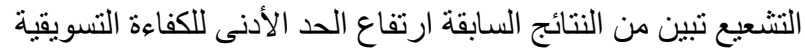

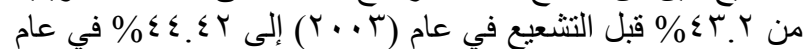

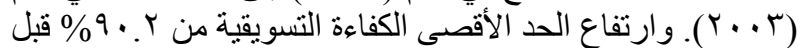

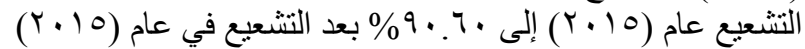

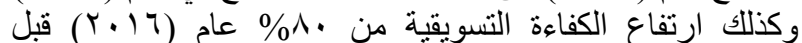

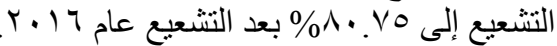

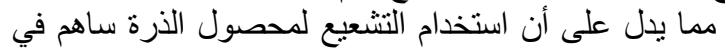
تحسين الكفاءة التسويقية لهذا المحصول حيث الخيث ساهم التشعيع في إطالة

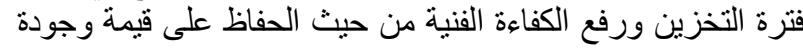

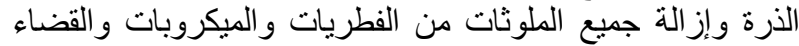

على الحثرات بها الأمر الذي يعني تحسين الجوات النب التسويقية

رابعاً: أثر استخدام التثعيع في تحسين الكفاعة التسويقية لمحصول البطاطس قبل وبعد إضافةٌة تكاليف التشعيع في مصر.

1- الهوامش التسويقية وتوزيع جنيه المستهلك لمحصول البطاطس قبل إضافة تكاليف التشعيع

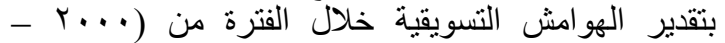

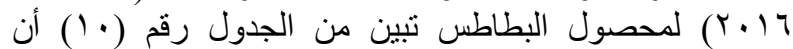

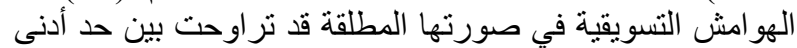

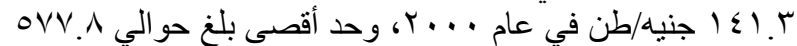

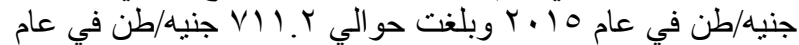

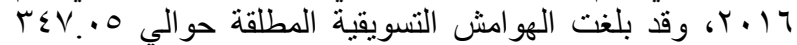

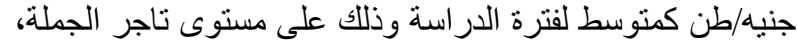

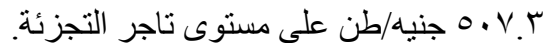

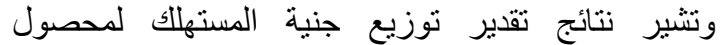

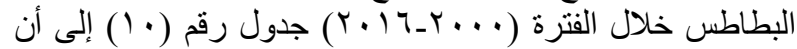

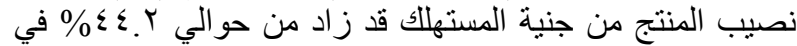

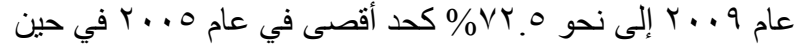

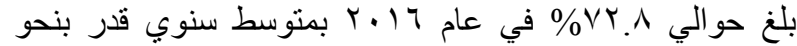

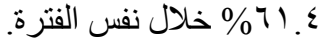

أما نصيب الوسطاء من جنية المستهلك فقد تذبذب النب حيث بلغ

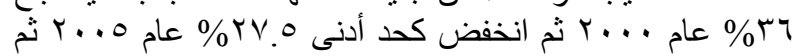

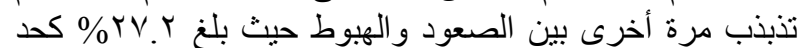

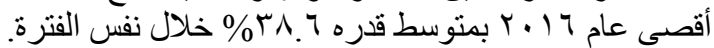

r- الهوامش التسويقية وتوزيع جنيه المستهلك لمحصول البطاطس بعد إضافة تكاليف التشعيع:

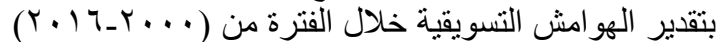

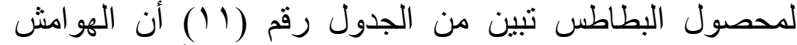

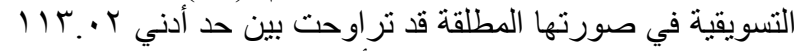

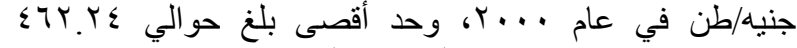

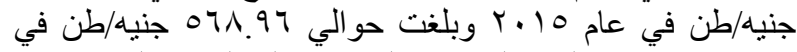

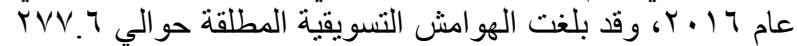
جنيه/طن كمتوسط لفترة الدر اسة وذللك على مستوى تاجر الجنه الجملة،

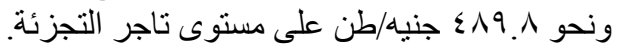

كما تبين من در اسة الكفاءة التسويقية وجود علاقة طردية مؤكدة احصائياً بين كل من الكفاءة التسويقية كمتغير تابع (E)

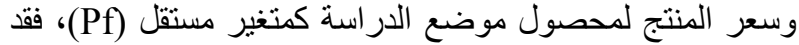

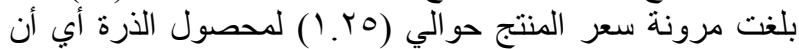

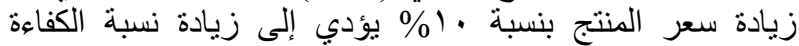

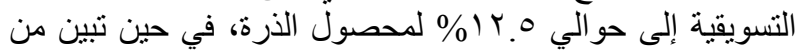

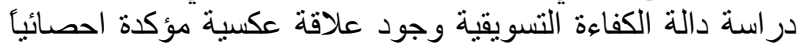

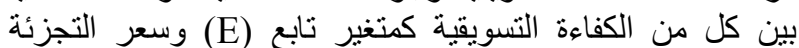

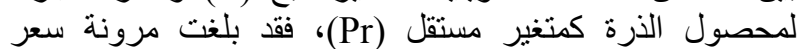

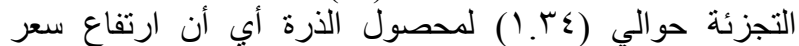

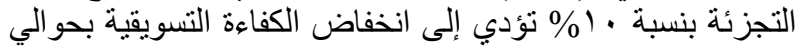
ع.با

كما تبين من دراسة دالة الكفاءة التسويقية وجود علاعة علاقة طردية بين كل من الكفاءة التسويقية كمتغير تابع وتكلفة إنتاج الطن

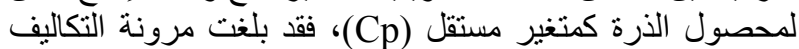
حوالي ( • • . • ) لمحصول كلغيرل الذرة.

أوضحت النتائج في الجدول رقم (^) أن الكفاءة التسويقية

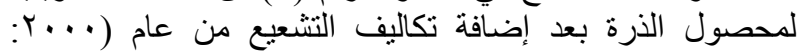

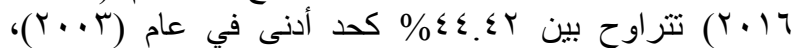

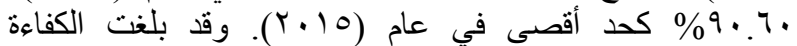

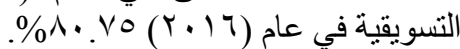

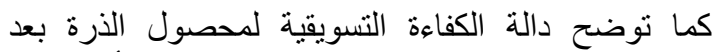

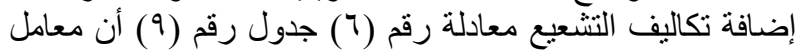

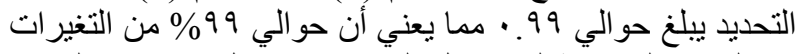

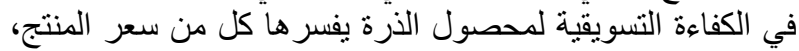

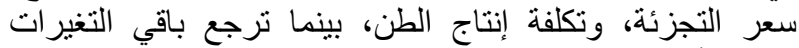
لعو امل أخرى لم تشملها دالة الكفاءة التسويقية.

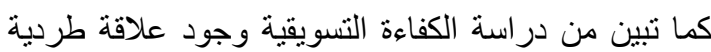
مؤكدة احصائياً بين كل من الكفاءة التسويقية كمنغير تابع (E)

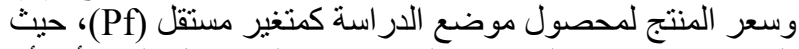

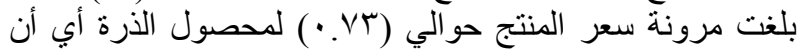

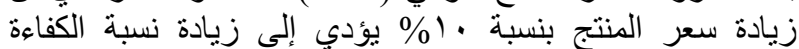

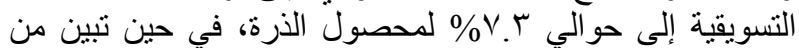
در اسة دالة

الكفاءة التسويقية وجود علاقة عكسية مؤكدة احصائياً بين كل

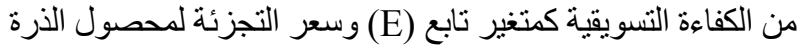

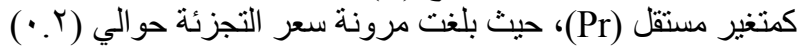

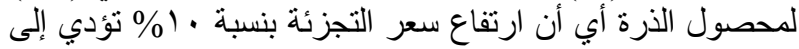

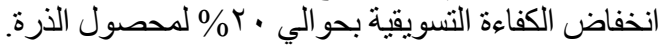

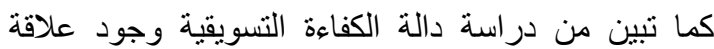
طردية بين كل من الكفاءة التسويقية كمتغير تابع وتكلفة دالة إنتاج الطن

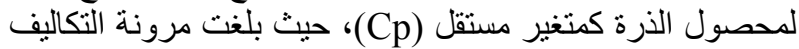

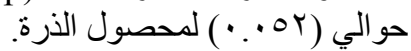




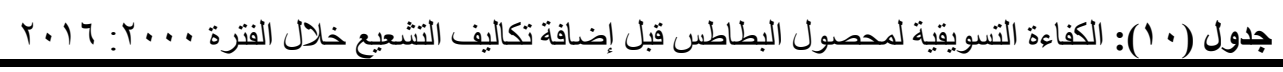

\begin{tabular}{|c|c|c|c|c|c|c|c|c|c|c|c|c|c|c|c|}
\hline \multirow{3}{*}{$\begin{array}{c}\text { التسويقية } \\
\text { \% }\end{array}$} & \multirow{3}{*}{$\begin{array}{c}\text { الوسطباء } \\
\text { \% }\end{array}$} & \multirow{3}{*}{ تاجزئ } & \multirow{3}{*}{ نصيب } & \multirow{3}{*}{ المنتج } & \multicolumn{2}{|c|}{ الانتشار السعرى } & \multicolumn{4}{|c|}{ الهوامش التسويقية } & \multirow{3}{*}{ جنيه/طن } & \multirow{3}{*}{ جنيه/طن الجملة } & \multirow{3}{*}{ جنيه/طن } & \multirow{3}{*}{ تكاليف } & \multirow{3}{*}{ السنوات } \\
\hline & & & & & \multirow[b]{2}{*}{ النسبى } & \multirow[b]{2}{*}{ المطلق } & \multicolumn{2}{|c|}{ هامش التجزئة } & \multicolumn{2}{|c|}{ هامش الجملة } & & & & & \\
\hline & & & & & & & النسبيش(") & المطلق المامثل) & النسبيى\%) & المطلق ('المامق & & & & & \\
\hline$\leqslant 9.9$ & r.. & $r \cdot$. & 17. & $T \leqslant$. & ז." & MIV.q & $r \cdot$. & 187.7 & 17. & $1 \leq 1 . r$ & ANז & $V \cdot T$ & 070 & T17 & $r \ldots$ \\
\hline$\varepsilon r . \varepsilon$ & $\varepsilon r . r$ & $r V . \wedge$ & $1 \leqslant . \varepsilon$ & ov.A & $\varepsilon r . r$ & $\varepsilon V Y . T$ & $r V_{. \wedge}$ & "11. & $1 \leqslant . \varepsilon$ & 171.7 & 1119 & $\Lambda \cdot \Lambda$ & $7 \leq 7$ & rדז & $r \ldots r$ \\
\hline$\leq 9.9$ & $r{ }^{\prime} \cdot$ & $r \cdot$. & 17. & $T \varepsilon$. & ד." & rov.o & $r \cdot$. & 191.7 & 17. & 101.9 & 994 & Vq & דזד & rot & $r \ldots r$ \\
\hline r.^.A & or. s & $\leqslant 1 . \wedge$ & 11.7 & $\leq 7.7$ & or. s & $v \leqslant 1 . \wedge$ & $\leqslant 1 . \wedge$ & $0 \wedge \cdot$. & 11.7 & 171.1 & $1 \% \wedge 9$ & 1.9 & $T \leq V$ & & $r \ldots r$ \\
\hline$\leq 9.9$ & ז." & $r \cdot$. & 17. & $T \varepsilon$. & r.. & $0 \leq \cdot V$ & $r \cdot$. & r... & 17. & $r \varepsilon \cdot r$ & $10 . r$ & $M \cdot r$ & 971 & ors & $r \ldots \varepsilon$ \\
\hline 09.7 & rV.O & $9 . \varepsilon$ & 11.1 & VY.O & rV.o & TI.Y & $9 . \varepsilon$ & Irs.. & 11.1 & $r \varepsilon \cdot r$ & DTO & $1 K \cdot 1$ & 971 & ors & $r \ldots o$ \\
\hline$\leq 9.9$ & r.. & $r \cdot \cdot$ & 17. & $T \leq$. & r." & $71 . V$ & $r \cdot$. & $r \leqslant r .7$ & 17. & $r V \leq .1$ & MIT & irv. & 1.97 & $7 \leq$ & $r \ldots r$ \\
\hline$\varepsilon 1$. & $\leq \varepsilon .7$ & $r \cdot . \wedge$ & $1 \pi . \wedge$ & $00 . \varepsilon$ & $\varepsilon \varepsilon .7$ & 170.7 & $r \cdot . \wedge$ & ०१V. & $1 T .1$ & r.^.7 & $19 \varepsilon$. & $1 T \leqslant r$ & $1 \cdot v \varepsilon$ & $T \cdot r$ & $r \ldots r$ \\
\hline$\leq 9.9$ & $r{ }^{\prime}$. & $r \cdot$. & 17. & $T \leq$. & דז. & $v \leqslant r . \leqslant$ & $r \cdot$. & 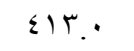 & 17. & s. & r. 70 & 1704 & ITY & $V \varepsilon$. & $r \ldots \wedge$ \\
\hline$r \cdot . \wedge$ & 00.1 & $\varepsilon \varepsilon . V$ & 11.1 & $\leq \varepsilon . r$ & 00.1 & M.人 & $\varepsilon \varepsilon . V$ & $1 . \wedge 7$. & 11.1 & 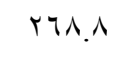 & $r \leqslant r$. & $\mid r \leq \varepsilon$ & 1.10 & $T . r$ & $r \ldots q$ \\
\hline$\leq 7.9$ & r." & $r \cdot$. & 17. & $T \varepsilon$. & r.. & $q \cdot V . r$ & $r \cdot$. & $0 . \varepsilon_{.}$ & 17. & $\varepsilon \cdot r . r$ & ror. & $r .17$ & $171 \pi$ & $\Lambda \cdot r$ & $r \cdot 1 \cdot$ \\
\hline$\leq \varepsilon .9$ & $\varepsilon \cdot . \wedge$ & rı. & $1 \leqslant .1$ & $09 . r$ & $\varepsilon \cdot \wedge$ & $1101 . r$ & rт. & VTV.V & $1 \leqslant .1$ & $\varepsilon r \cdot . T$ & $r \wedge \varepsilon l$ & $r) \cdot r$ & ITAr & $q \leq r$ & $r .11$ \\
\hline$\leq 9.9$ & ז." & $r \cdot . \cdot$ & 17. & $T \leqslant$. & r.. & $1.99 . \varepsilon$ & $r \cdot$. & $\pi \cdot . \wedge$ & 17. & $\varepsilon \wedge \wedge .7$ & $r .0 \leqslant$ & $r \leq \varepsilon r$ & 1900 & 1.90 & $r \cdot I r$ \\
\hline$\leq 7 . \varepsilon$ & r & $r \leqslant . r$ & 10.9 & $7 \cdot . V$ & rq. & Irso. & $r \leqslant . r$ & $\vee \wedge 9 . \Sigma$ & $10 . r$ & $\leq 90.7$ & & $r \leq V \wedge$ & I 914 & 111. & $r \cdot 1 r$ \\
\hline$\leq 9.9$ & r." & $r \cdot \cdot$ & 17. & $T \leq$. & rq. & kor.l & $r \cdot$. & 797.1 & 17. & 007.9 & $r \leqslant \lambda l$ & rVAO & TYYA & $I r \leqslant V$ & $r \cdot I \leq$ \\
\hline$\varepsilon \Lambda . r$ & $r v . \varepsilon$ & r..A & 10.7 & Tr.T & rV. $\varepsilon$ & Irkr.q & YI.A & 1.0 .1 & 10.7 & orv.A & M79s & rANq & सTII & $1 Y q \leq$ & $r \cdot 10$ \\
\hline $7 \cdot$ & rV.r & 9. & $11 . r$ & $V Y . \wedge$ & TV.r & 1.74 .7 & 9. & rol. & $11 . r$ & $v 11 . r$ & $r q . v$ & ro07 & $r \wedge \leqslant 0$ & 1094 & $r .17$ \\
\hline$\Lambda \cdot r . \varepsilon$ & 707.9 & rqo.0 & $Y 4 \cdot . \wedge$ & $1 \cdot \leq r . \wedge$ & 907.4 & I \&Orr.V & $r q 0.0$ & Aฯrr.V & $r צ \cdot . \wedge$ & $0 \wedge 99.9$ & rAIrr & $r q \leq q q$ & $r r \Delta q q$ & 111. & الإجمالي \\
\hline$\sum V, Y Y$ & r^. & $r r . r$ & $10 . r$ & $71 . \varepsilon$ & rی. ${ }^{\prime}$ & $\Lambda 0 \leq$ & $r r . r$ & $0 . v . r$ & $10 . r$ & $r \leqslant V$ & $r Y \leq Y$ & IVTO & $\mid r \wedge \Lambda$ & VVI & المتوسط \\
\hline
\end{tabular}




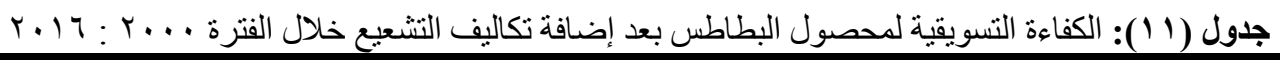

\begin{tabular}{|c|c|c|c|c|c|c|c|c|c|c|c|c|c|c|c|}
\hline \multirow{3}{*}{ التسويقية } & \multirow{3}{*}{$\begin{array}{c}\text { الوسطيب } \\
\text { \% }\end{array}$} & \multirow{3}{*}{$\begin{array}{c}\text { نصيب } \\
\text { تاجزئة } \\
\text { \% }\end{array}$} & \multirow{3}{*}{ نصاجز } & \multirow{3}{*}{ نصيب } & \multicolumn{2}{|c|}{ الانتثار السعرى } & \multicolumn{4}{|c|}{ الهوامش التسويقية } & \multirow{3}{*}{ جنيه/طن التجنة } & \multirow{3}{*}{ جنيه/طن الجملة } & \multirow{3}{*}{ جنيه/طن } & \multirow{3}{*}{ جنياليف/طن } & \multirow{3}{*}{ السنوات } \\
\hline & & & & & \multirow[b]{2}{*}{ النسبى } & \multirow[b]{2}{*}{ المطلق } & \multicolumn{2}{|c|}{ هامش التجزئة } & \multicolumn{2}{|c|}{ هامش الجملة } & & & & & \\
\hline & & & & & & & $\begin{array}{l}\text { النسامشى(") } \\
\text { \% }\end{array}$ & الهامشت (") & النسبيى\%) & الهامشت ('المطق & & & & & \\
\hline or.sr & r.^. & $r \cdot \ldots$ & Ir.A. & TV.Y. & r..A. & Y^९.T & $r . .$. & $1 \times 7.7$. & IY.A. & $11 r . r$ & גᄉr & $V \cdot T$ & $09 \pi$ & זמז & $r \ldots$ \\
\hline אז.r & ס ז. & YV.Vq & 11.00 & $7 . .70$ & ס ז.r & $\varepsilon \varepsilon \cdot r$ & YV.Vq & M.... & 11.00 & IYq.YA & 1119 & $\Lambda \cdot \Lambda$ & $7 \times 9$ & rᄉ. & $r \ldots l$ \\
\hline r. & r..A. & $r \cdot .$. & Ir.A. & TV.Y. & M.^. & MTO.V & $r \cdot .$. & 191.7 & IY.A. & IrV.1. & 994 & V৭ะ & TTV & $r v \varepsilon$ & $r \ldots r$ \\
\hline$r \leq .91$ & 01.11 & $\leqslant 1 . V 7$ & Q.Tr & $\leqslant \Lambda .9 r$ & 01.11 & $v \cdot 9 . \varepsilon$ & «l.V & $0 \wedge . .$. & a.r & $1 \times 9 . \varepsilon \varepsilon$ & $1 \% \wedge q$ & 1.9 & 71. & rNi & $r \ldots r$ \\
\hline or. $\leqslant$ r & r.... & $r \cdot$. & Ir.A. & $T V . Y$. & r.... & $\leqslant q Y . V$ & $r \cdot . \cdot$ & r... & Ir.A. & 194.17 & $10 . r$ & $I r \cdot r$ & $1 \ldots 9$ & 070 & $r \ldots \varepsilon$ \\
\hline$T \leq .1 T$ & rT.AT & 9.47 & $1 \leq .0$. & $V 7.1 \leq$ & rT.AT & rIT.r & 9.47 & $\mid r \leq \ldots$ & $1 \leq .0$. & 194.17 & IrTo & $\mid r \cdot 1$ & $1 \ldots 9$ & 070 & r... \\
\hline r. & r..A. & $r \cdot .$. & IY.A. & TV.Y. & rY.A. & 071.9 & $r \cdot .$. & $r \leqslant r .7$. & IY.A. & Y) & IVIT & Irv. & 1101 & $T \leqslant 0$ & $r \ldots r$ \\
\hline$\varepsilon r . \vee \tau$ & $\leqslant 1.10$ & $r \cdot . V V$ & 11.11 & 01.10 & $\leqslant 1.10$ & 111.9 & $r \cdot . v V$ & oqv... & 11.11 & $Y I \leqslant . \wedge \Lambda$ & $19 \leq$. & $1 \pi \leq r$ & $11 \mathrm{rA}$ & TrT & $r \ldots v$ \\
\hline or. $\leqslant$ r & r..A. & $r \cdot . \cdot$ & Ir.A. & $T V . Y$. & r.... & TVV.T & $r \cdot . \cdot$ & $\varepsilon 1 т .$. & Ir.A. & rTE. Y T & $r .70$ & $170 r$ & ITAN & VVV & $r \cdots \lambda$ \\
\hline rr.v. & or.os & $\varepsilon \leqslant .79$ & 1.10 & $\leq 7, \leqslant 7$ & or.os & $1+\cdot 1$. & $\leqslant \leqslant .79$ & $1 . \wedge 7 .$. & 1.10 & rlo. & $r \leqslant r$. & $1 \pi \leq \varepsilon$ & 1149 & אזד & $r \ldots q$ \\
\hline $0 . . \leqslant V$ & r.... & $r \cdot . \cdot$ & Ir.A. & $T V . Y$. & r.... & ArT.T & $r \cdot . \cdot$ & $0 . \varepsilon \ldots$ & Ir.A. & MrY.0T & ror. & $r .17$ & 1794 & $\Lambda \leq r$ & $r \cdot 1$. \\
\hline$\leq V .9 \leq$ & rV.AI & ro.9V & 11.10 & 97.19 & rV.Al & $1 \cdot V \varepsilon . r$ & ro.9V & VTV.TA & 11.10 & ג צזיד & rᄉミl & $r) \cdot r$ & IVTV & 919 & $r .11$ \\
\hline or. $\leqslant$ r & r..A. & $r \cdot$. & Ir.A. & $T V . Y$. & r.... & $1 \ldots 1 . v$ & $r \cdot$. & $71 . \wedge$. & Ir.A. & $r q . . q r$ & r. $0 \leqslant$ & $r \leq \leqslant r$ & r.or & $11 \leq 9$ & $r \cdot I r$ \\
\hline$\leqslant 9.0 \mathrm{~V}$ & r...9 & $r \leq .17$ & M.IT & Tr.VI & r...9 & 1110.1 & $r \leq .17$ & V^q. rV & $1 Y .1 T$ & r97. $\leqslant \wedge$ & Trty & $r \leqslant V A$ & $r \cdot N r$ & 1177 & $r \cdot 1 r$ \\
\hline or. $\leqslant$ r & r..A. & $r \cdot$. & Ir.A. & $T V . Y$. & r.... & $11 \leq 1 . V$ & $r \cdot$. & $797.1 \leq$ & Ir.A. & $\varepsilon \leqslant 0.0 r$ & $r \leq \lambda 1$ & rVAO & qruq & $|\pi|$. & $r \cdot 1 \leq$ \\
\hline 01.10 & $r \varepsilon . r$ & YI.Vq & $1 Y .01$ & 70.79 & $r \leqslant . r$ & IrTV.r & YI.Vq & 0.1 .7 & $1 Y .01$ & $\varepsilon T Y . Y \varepsilon$ & Tา9ะ & rA^q & $r \leqslant r V$ & 1509 & $r .10$ \\
\hline $7 \leq .01$ & $r T .00$ & 1.99 & $1 \leq .07$ & $V 7 . \leqslant 0$ & $r T .00$ & $q r \cdot . \varepsilon$ & 1.99 & rol. $\leqslant$. & $1 \leq .07$ & 071.97 & $r q . v$ & roor & YQAV & ITVT & $r .17$ \\
\hline$\wedge \neg \cdot . \vee \vee$ & $7 . \varepsilon$. & rq०.r^ & $r \cdot \Lambda . v 0$ & 1.90 .97 & $7 . \varepsilon . \cdot \varepsilon$ & $I r r \leq r . V$ & rq०.r & Arr & $r \cdot \Lambda . v_{0}$ & $\leqslant V Y \cdot .19$ & rNIrr & $r q \leq q q$ & $r \leqslant \vee \wedge$. & IrVvi & الإجمالي \\
\hline 0.09 & ro.0 & rr.r & Ir.rA & $7 \leqslant . \leqslant V$ & ro.0 & $\vee \wedge \varepsilon .9$ & rr.r & $\leqslant \wedge ৭ . \wedge$ & IY.YA & rVV.7 & $r Y \leq r$ & IVTo & $1 \leq 0 V$ & $\wedge 1$. & المتوسط \\
\hline
\end{tabular}


حين تبين من دراسة دالة الكفاءة التسويقية وجود علاقة عكسية مؤكدة احصائياً بين كل من الكفاءة التسويقية كمتغير تنابع (E)

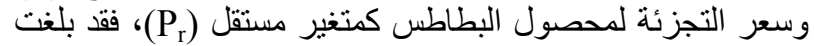

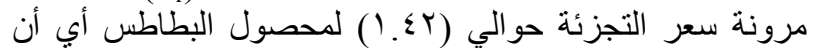

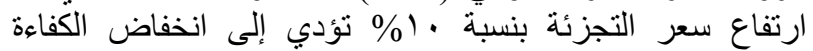

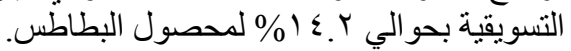

كما تبين من دراسة دالة الكفاءة التسويقية وجود علاقة طردية بين كل من الكفاءة التسويقية كمتغير تابع وتكلفة إنتاج الطن الطن

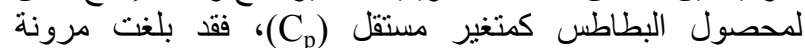

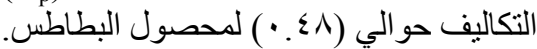

أوضحت النتائج في جدول رقم (11) أن الكفاءة التسويقية

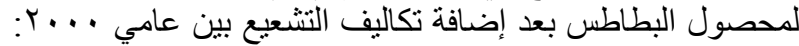

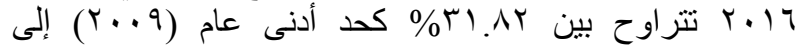

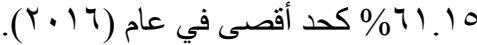

كما توضح دالة الكفاءة التسويقية لمحصول البطاطس بعد

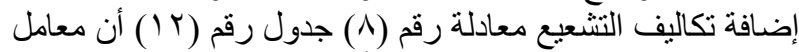

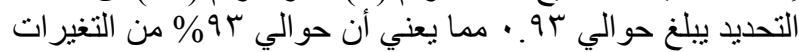

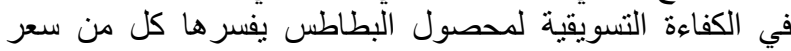

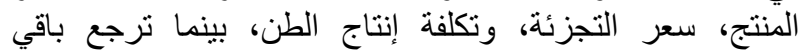
التغيرات لعو امل أخرى لم تشملها دالة الكفاءة التسويقية.

حيث تبين من دراسة الكفاءة التسويقية وجود علاقة طردية مؤكدة احصائياً بين كل من الكفاءة التسويقية كمتغير تنابع (E)

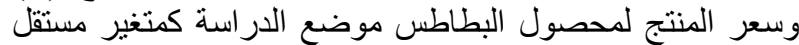

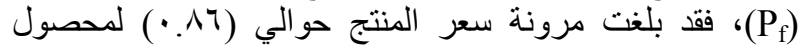

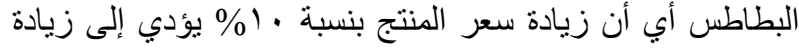

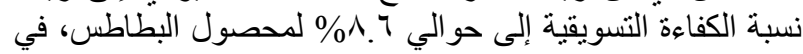
حين تبين من دراسة دالة الكفاءة التسويقية وجود التهاءة علاقة عكسية

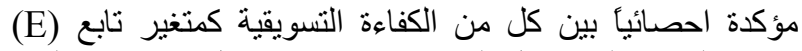

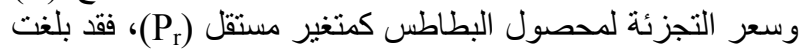

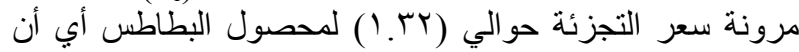

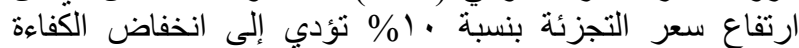

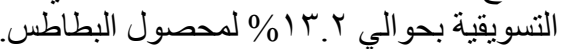

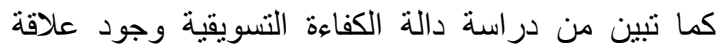
طردية بين كل من الكفاءة التسويقية كمتغير تنابع وتكلفة إنتاج الطن الطن

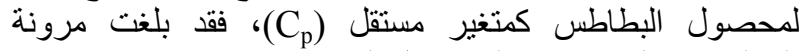
التكاليف حو الي (r • • ) لمحصول البطير البطاطس.

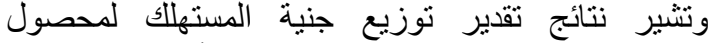

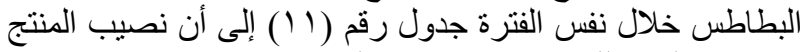

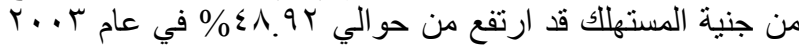

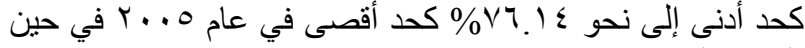

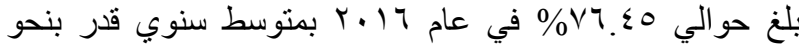

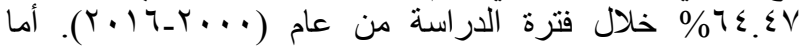

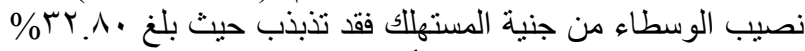

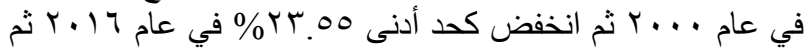

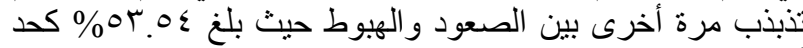

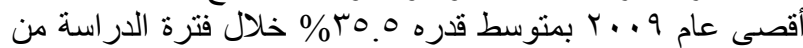

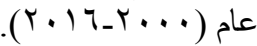

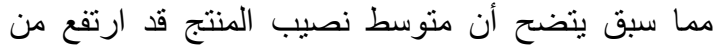

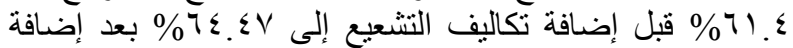
تكاليف التشعيع من جنيه المستهلك لمحصول البطاطس خلال

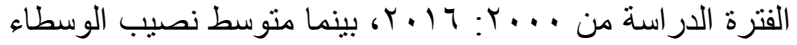

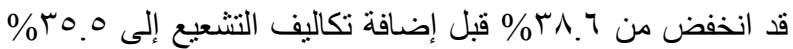

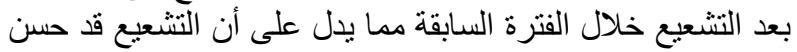
من نصيب المنتج وحد من نصيب الوسطاء.

r- العوامل المؤثرة على الكفاءة التسويقية لمحصول البطاطس قبل إضافة تكاليف التشعيع:

أوضحت النتائج في جدول رقم (• (1) أن الكفاءة التسويقية

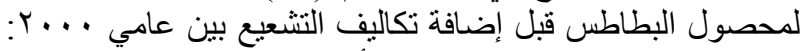

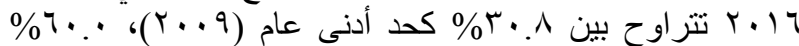

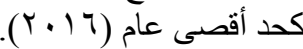

وتوضح دالة الكفاءة التسويقية لمحصول البطاطس قبل

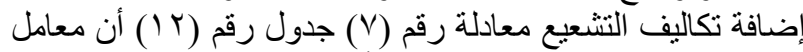

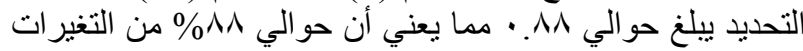

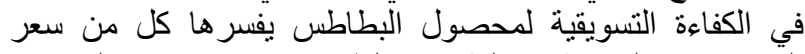

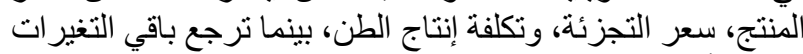
لعو امل أخرى لم تشملها دالة الكفاءة التسويقية.

حيث تبين من در اسة الكفاءة التسويقية وجود علاقة طردية مؤكدة احصائياً بين كل من الكفاءة التسويقية كمتغير تابع (E) وسعر المنتج لمحصول البطاطس موضع الدون الدراسة كمتغير مستقل

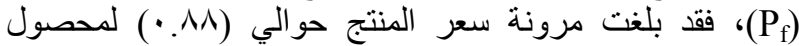

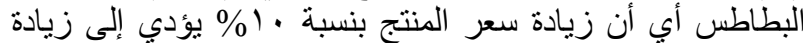

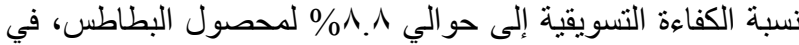

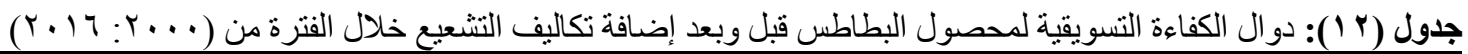

\begin{tabular}{|c|c|c|c|}
\hline $\mathbf{R}^{-2}$ & $\mathbf{F}$ & المعادلة & التغتير \\
\hline 0.88 & $33.9^{* * *}$ & $\begin{array}{l}\mathrm{E}=49.11+0.03 \mathrm{P}_{\mathrm{f}}-0.03 \mathrm{P}_{\mathrm{r}}+0.03 \mathrm{C}_{\mathrm{p}} \rightarrow(7) \\
\quad(26.62)^{* * * *}(3.18)^{* * *}(-9.25)^{* * *}(1.86)^{* * *}\end{array}$ & قابل تكايفة التسويقية \\
\hline 0.93 & $55.74^{* * *}$ & $\begin{aligned} \mathrm{E}= & 54.95+0.03 \mathrm{P}_{\mathrm{f}}-0.03 \mathrm{P}_{\mathrm{r}}+0.002 \mathrm{C}_{\mathrm{p}_{* * *}} \rightarrow(8) \\
& (12.82)^{* * *}(7.09)^{* * *}(-11.87)^{* * *}(3.07)^{* * *}\end{aligned}$ & بعد الكاءة التسويقية \\
\hline
\end{tabular}

(ألمصدر: جدول (·) (1) (1)

لمحصول البطاطس ساهم في تحسين الكفاءة التسويقية لهذا التهاء

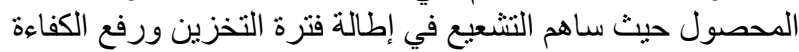

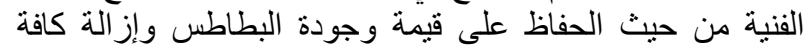

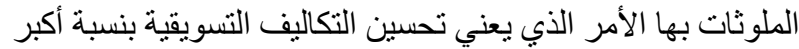
من التحسين في التكاليف الإنتاجية.

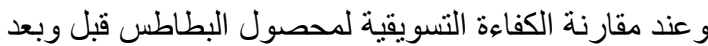
التشعيع تبين من النتائج السابقة ارتفاع الحد الأدنى للكفاءة التسويقية

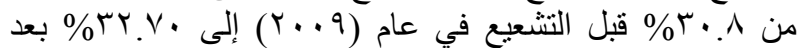

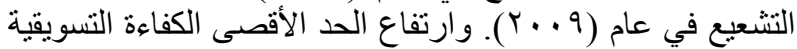

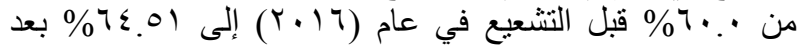

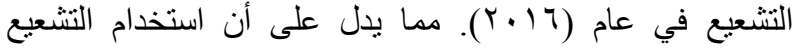




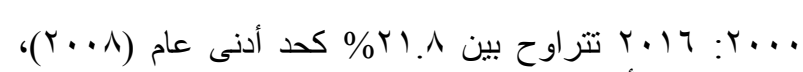

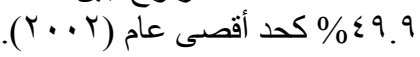

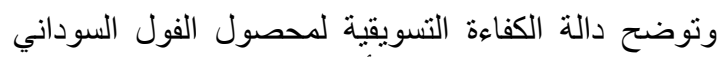

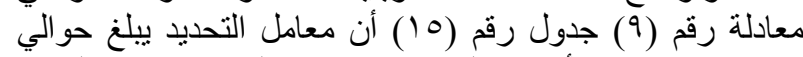

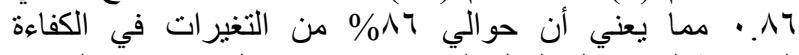

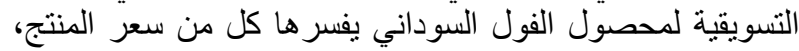

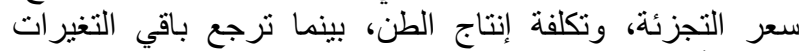
لعو امل أخرى لم تثنملها دالة الكفاءة التنسويقية.

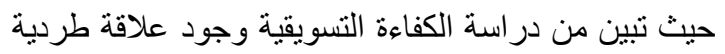

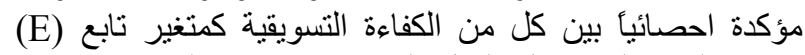

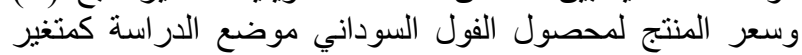

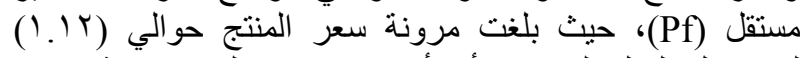

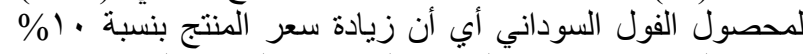

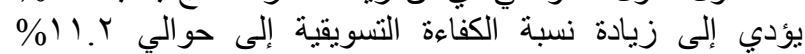

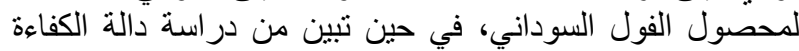

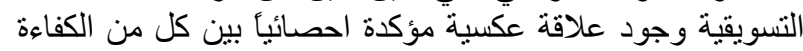

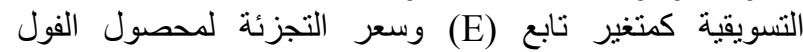

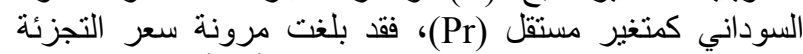

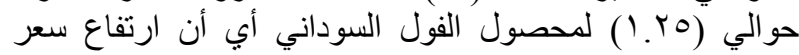

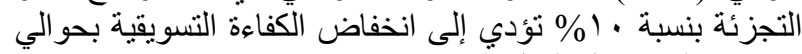

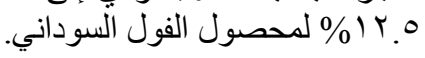

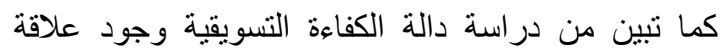

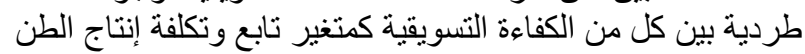

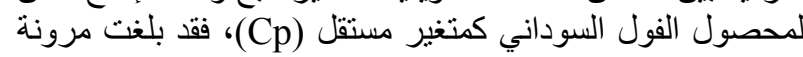

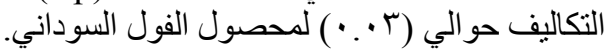

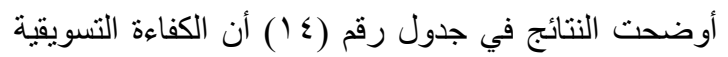

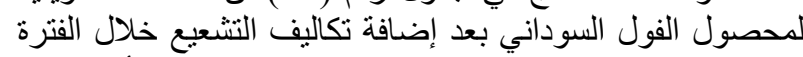

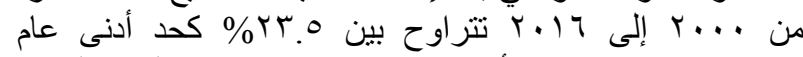

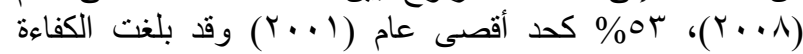

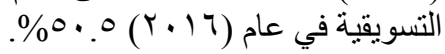

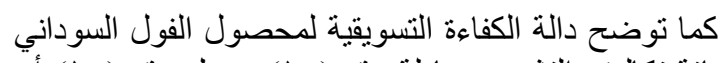

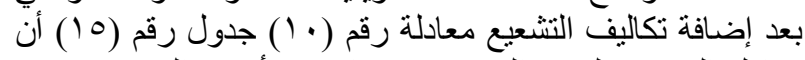

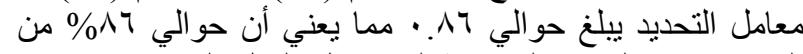

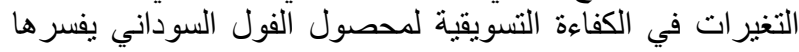

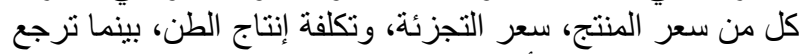

باقي التغير ات لعوامل أخرى لم تنشملها دالة الكفاءة التسويقية.

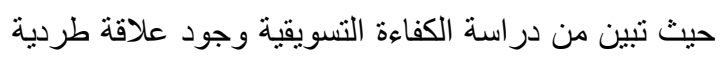

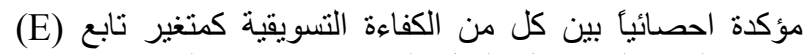

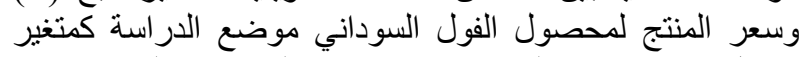

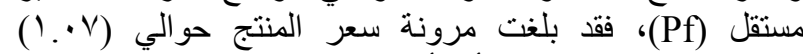

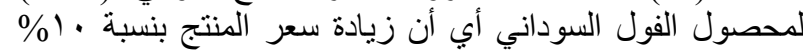

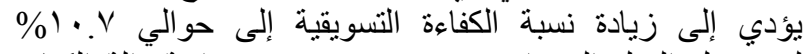

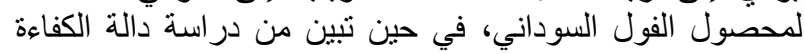

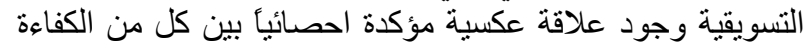

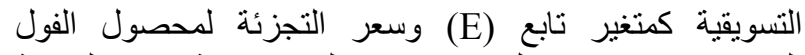

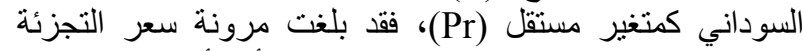

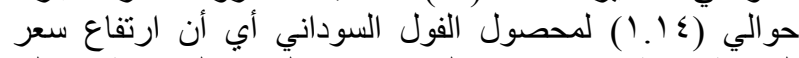

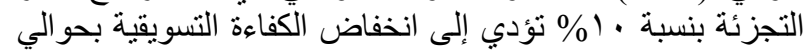

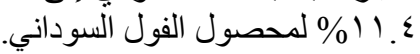

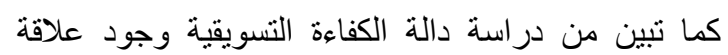

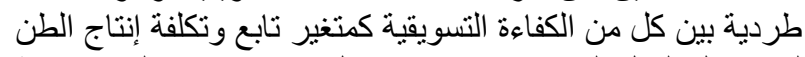

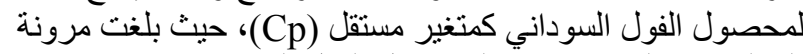

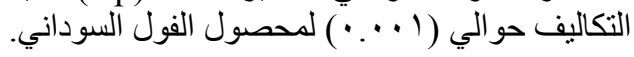

خامساً: أثر استخدام التشعيع في تحسين الكفاءة التسويقية لمحصول الفول السودانيقبل ويعد إضافة تكاليف التشعيع في مصر.

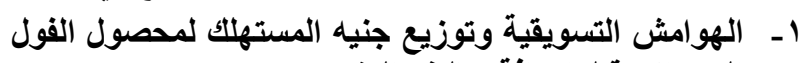
السوداني قبل إضافة تكاليف التشعيع.

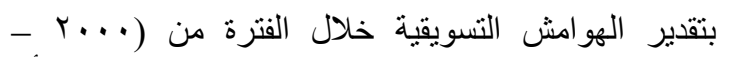

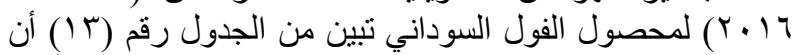

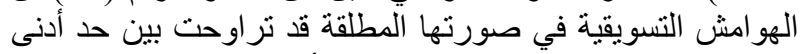

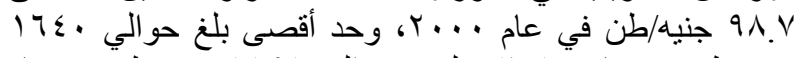

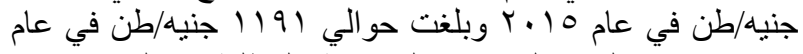

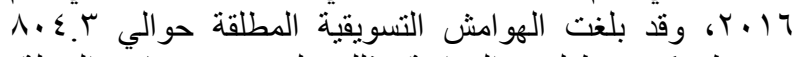

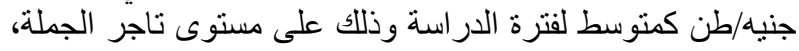

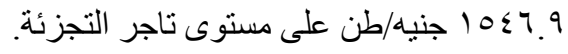

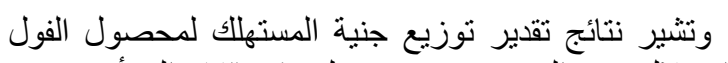

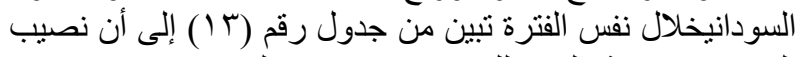

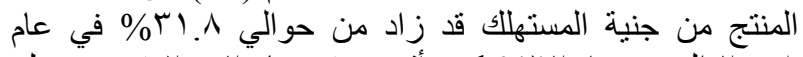

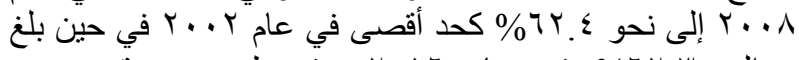

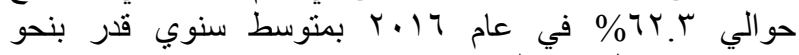

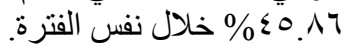

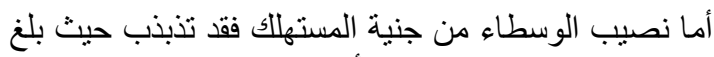

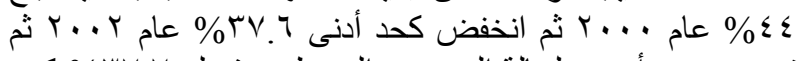

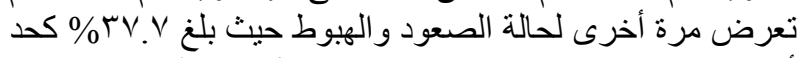

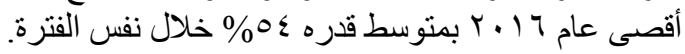

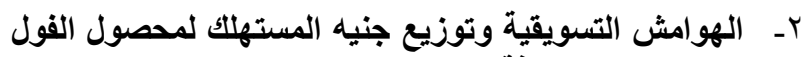
السوداني بعد إضافة تكاليف التشعيع:

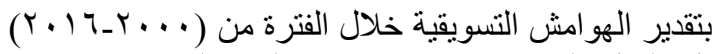

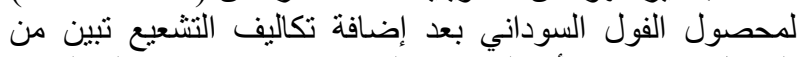

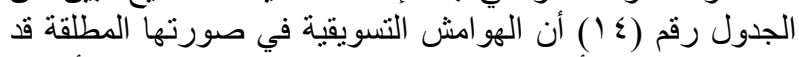

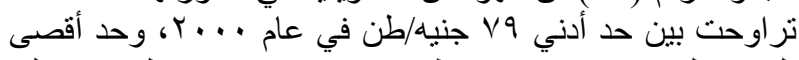

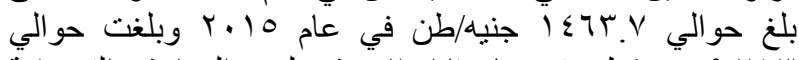

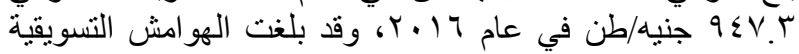

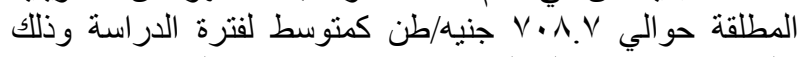

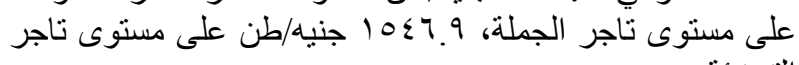

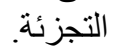

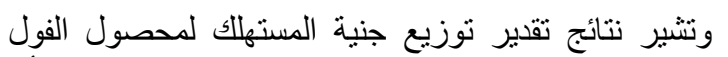

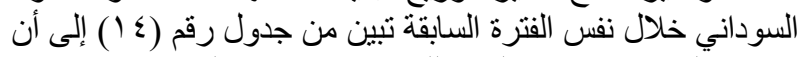

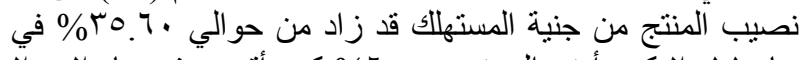

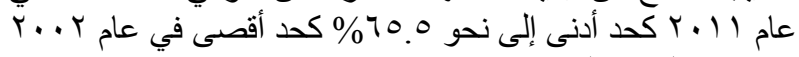

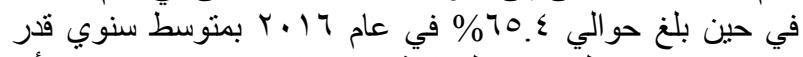

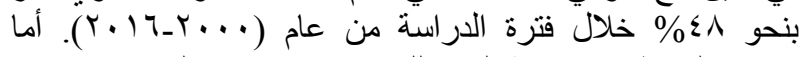

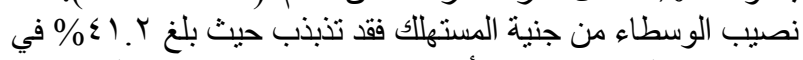

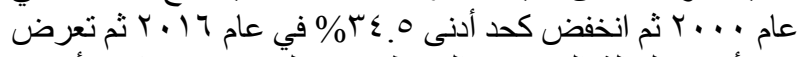

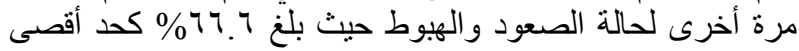

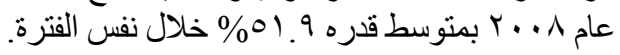

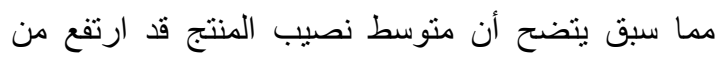

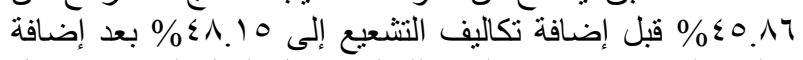

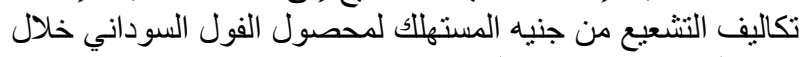

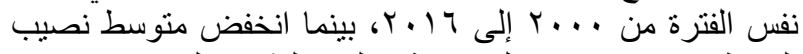

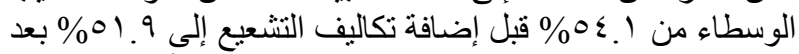

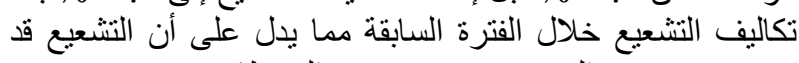
حسن من نصيب المنتج وحد من نصيب الوسطاء.

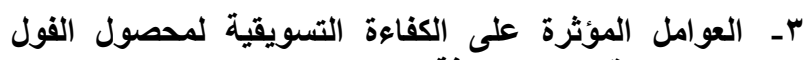
السوداني قبل وبعد إضافة تكاليف التشبيع:

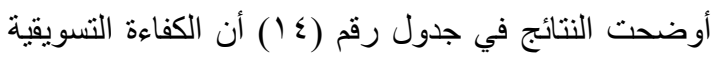
لمحصول الفول السوداني بعد إضافة تكاليف التشعيع بين عامي النيانية 


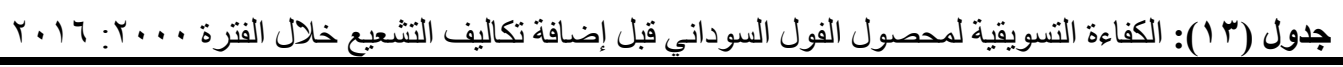

\begin{tabular}{|c|c|c|c|c|c|c|c|c|c|c|c|c|c|c|c|}
\hline \multirow{3}{*}{$\begin{array}{c}\text { التسويقية } \\
\text { \% }\end{array}$} & \multirow{3}{*}{$\begin{array}{c}\text { نصيب } \\
\text { الوسطاء }\end{array}$} & \multirow{3}{*}{ تصاجزيب } & \multirow{3}{*}{ نصيب تاجر } & \multirow{3}{*}{ المنتج } & \multicolumn{2}{|c|}{ الانتشار السعرى } & \multicolumn{4}{|c|}{ الهوامش التسويقية } & \multirow{3}{*}{ جنيه/طن } & \multirow{3}{*}{ جنيه/طن } & \multirow{3}{*}{ جنيه/طن } & \multirow{3}{*}{ جنيالة/طناج } & \multirow{3}{*}{ السنوات } \\
\hline & & & & & \multirow[b]{2}{*}{ النسبى } & \multirow[b]{2}{*}{ المطلق } & \multicolumn{2}{|c|}{ هامش التجزئة } & \multicolumn{2}{|c|}{ هامش الجملة } & & & & & \\
\hline & & & & & & & النسبيى\%) & المامشلق المطلق & 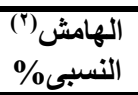 & المهلق('املق & & & & & \\
\hline$\varepsilon r . r$ & $\varepsilon \varepsilon$. & $r \cdot$. & $1 \varepsilon$. & 07. & $\varepsilon \varepsilon$. & $r \cdot \cdot r$ & $r \cdot$. & $r 11.0$ & $1 \leqslant$. & $9 \wedge . \vee$ & $V .0$ & $\leq 9 \leq$ & $r 90$ & rrV & $r \ldots$ \\
\hline$\varepsilon \Lambda . r$ & rq.1 & 19.7 & 19.7 & $7 . .9$ & rq.1 & $\leq 0 .$. & 19.7 & rro. & 19.7 & rro. & 110. & qro & $v \ldots$ & $\varepsilon r$. & $r \ldots 1$ \\
\hline$\leq 9.9$ & rV.T & r.. & 10.7 & Tr. & rv. T & $099 . \wedge$ & r.. & rol." & 10.7 & $r \leq \Lambda . \wedge$ & 1090 & $1 T \leq \varepsilon$ & 990 & $09 V$ & $r \ldots r$ \\
\hline$\leqslant r_{0} 0$ & $\varepsilon \varepsilon .9$ & $r \cdot$. & $1 \leqslant .9$ & 00.1 & $\leqslant \varepsilon .9$ & 910. & $r \cdot$. & TI. & $1 \leqslant .9$ & $r \cdot r$. & $r \cdot \varepsilon$. & $1 \leq r \wedge$ & 11 ro & TVO & $r \ldots r$ \\
\hline$r \cdot r$ & ov. 9 & $r v .1$ & $r \cdot . \wedge$ & $\leq r_{.} 1$ & ov. 9 & $1 \leq \varepsilon$. & rv.l & qrr. & $r \cdot . \wedge$ & 011. & $r \leqslant 10$ & r & $1 . \leqslant 0$ & THV & $r \ldots \varepsilon$ \\
\hline TV.r & 71.7 & ro.A & ro.A & $r \wedge . \varepsilon$ & 7.7 & 11.0. & ro.A & $1 . \leqslant \Lambda_{.}$. & ro.A & vor. & тат. & IANT & 11 ro & TVo & $r \ldots o$ \\
\hline r. & Tr.V & $r \cdot$. & Tr.v & $r v . r$ & Tr.V & rIV. & $r \cdot$. & 1.15 .0 & rT.V & $11 . \leqslant 0$ & Troo & אדיזי & IrON & voo & $r \ldots T$ \\
\hline$r \leqslant . r$ & $70 . r$ & $\varepsilon r . \varepsilon$ & r. $q$ & $r \varepsilon . V$ & $70 . r$ & $r \leqslant 90$. & $\varepsilon r . \varepsilon$ & 1719. & rr.q & 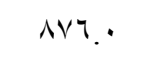 & rAr. & $r+1$ & ITro & 190 & $r \ldots v$ \\
\hline YI.A & $T \Lambda . r$ & $\varepsilon \cdot .9$ & rV.r & ז.^ & $T \Lambda . r$ & rq1... & $\varepsilon \cdot .9$ & $1 v \leqslant 0$. & rV.r & 1170. & STro & ror. & 1700 & או & $r \ldots \lambda$ \\
\hline r^. & 7.7 & rq. & $r . r$ & rq. & $7 . .7$ & rNor." & rq. & $110 \leqslant$. & r). r & $1 \ldots r$. & $\leq v 1$. & rNoT & $110 \leqslant$ & $111 \mathrm{r}$ & $r \ldots q$ \\
\hline$r \leqslant$. & 70.0 & $\varepsilon \varepsilon .9$ & $r \cdot .0$ & $r \varepsilon .0$ & 10.0 & rrvo." & $\leqslant \varepsilon .9$ & rMIT. & $r \cdot .0$ & 1.09. & 0100 & rArq & IVA. & 1.71 & $r \cdot 1$. \\
\hline rr.o & 7. & $\varepsilon r . \tau$ & r.to & 9 & 77.1 & rv. ... & $\varepsilon r . \tau$ & $r \leqslant \varepsilon r$. & r.Po & irtr. & 0т.. & T101 & 11997 & Nא & $r \cdot 11$ \\
\hline ro.s & or.r & $r \cdot \cdot$ & rY. & $\leq \vee . \wedge$ & or.r & mon. & $r \cdot \cdot$ & 1N1r.0 & TY. & $\mu \leq \varepsilon .0$ & $7 . \leq 0$ & STr & YAAV & IVT & $r \cdot 1 r$ \\
\hline$r \varepsilon . \varepsilon$ & or.s & $\leqslant 7 . \varepsilon$ & $v_{.} \cdot$ & $\leq 7.7$ & or. & $r \leq 70$. & $\leqslant 7 . \varepsilon$ & $r .1 r$. & $v_{.} \cdot$ & sor. & $7 \leq 9$. & $r \leq V V$ & r. ro & 1110 & $r \cdot 1 T$ \\
\hline r. & $01 . \varepsilon$ & $\leq 0 . r$ & $7 . r$ & $\leq \Lambda . T$ & $01 . \varepsilon$ & r074. & $\leq 0 . r$ & rाrq. & $7 . Y$ & $\varepsilon r V_{.}$. & 7970 & rVaT & 9דושי & $r \cdot r_{1}$ & $r \cdot 1 \leq$ \\
\hline ro.s & Or.r & $r \cdot \cdot$ & TY. & $\leq V_{.} \wedge$ & or.r & rᄉos. & $r \cdot \cdot$ & TYIE. & TY. & $17 \varepsilon \cdot$ & VTA. & 0177 & TOYt & 5117 & $r .10$ \\
\hline$\leq 9 . \wedge$ & $r v . V$ & $r r_{0} 0$ & $10 . r$ & r.r & rv.v & r90... & $r r_{0} 0$ & 1809. & $10 . r$ & 1191. & vAro & 7.79 & $\leqslant \wedge V_{0}$ & raro & $r .17$ \\
\hline$\Delta \wedge \cdot .0$ & $q r \cdot . \varepsilon$ & $0 \wedge 9.9$ & & $\vee \vee 9.7$ & $q \uparrow \cdot . \varepsilon$ & rqqv. & $0 \wedge 9.9$ & ห৭ห१५.० & $r r \cdot . v$ & irgvr.o & VYO.O & \&qr). & rYoro & $19 Y \wedge \varepsilon$ & الإجمالي \\
\hline$r \varepsilon .1 r$ & O & $r \varepsilon . V$ & $19 . \leqslant 0$ & $\leqslant 0 . \wedge 4$ & $0 \leqslant$ & rrol & $r \varepsilon . V$ & $10 \leq 7.9$ & 19.50 & $\Lambda \cdot \varepsilon$ & \& r & rVIA & 1914 & ع & المتوسط \\
\hline
\end{tabular}

(1) الهامش المطلق (التجزئة) = سعر التجزئة - سعر المنتج

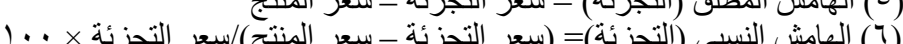

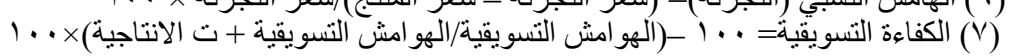

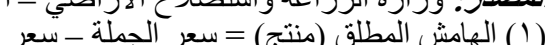

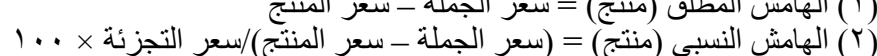

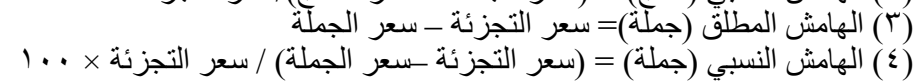




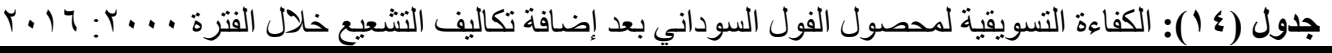

\begin{tabular}{|c|c|c|c|c|c|c|c|c|c|c|c|c|c|c|c|}
\hline \multirow{3}{*}{ التسويقية } & \multirow{3}{*}{$\begin{array}{c}\text { نصبيب } \\
\text { الوسطاء }\end{array}$} & \multirow{3}{*}{$\begin{array}{c}\text { نصيب تاجزة } \\
\text { التجزئة } \\
\text { \% }\end{array}$} & \multirow{3}{*}{ نصيب تاجز } & \multirow{3}{*}{$\begin{array}{l}\text { المنتج } \\
\text { \% }\end{array}$} & \multicolumn{2}{|c|}{ الانتثار السعرى } & \multicolumn{4}{|c|}{ الهوامش التسويقية } & \multirow{3}{*}{ جنيه/طنئة } & \multirow{3}{*}{ جنيه/طن } & \multirow{3}{*}{ جنيه/طن } & \multirow{3}{*}{ تكاليف الإنتاج } & \multirow{3}{*}{ السنوات } \\
\hline & & & & & \multirow[b]{2}{*}{ النسبى } & \multirow[b]{2}{*}{ 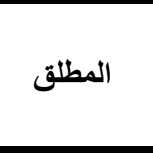 } & \multicolumn{2}{|c|}{ هامش التجزئة } & \multicolumn{2}{|c|}{ هامش الجملة } & & & & & \\
\hline & & & & & & & 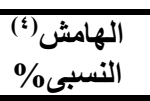 & المامشق المط") & النسبيى") & الهامش"'الق & & & & & \\
\hline or. & $\varepsilon 1 . r$ & $r \cdot$. & $11 . r$ & $0 \wedge . \wedge$ & $\varepsilon 1 . r$ & $r q . .0$ & $r \cdot$. & Y11.0 & $11 . r$ & $v^{\prime}$. & $v .0$ & $\leq 9 \leq$ & $\leqslant 10$ & MIV & r... \\
\hline $0 \leqslant .7$ & r. & 19.7 & 17.0 & 9.9. & T. & $\leqslant 10$. & 19.7 & rro. & 17.0 & $19 .$. & 110. & qro & VTO & $0 .$. & $r \ldots l$ \\
\hline $00 . r$ & $r \leqslant .0$ & r.. & Ir.o & 70.0 & $r \leqslant .0$ & $00 .$. & r.. & ro1. & $1 r .0$ & 199. & 1090 & IrEs & $1 . \leq 0$ & TVV & $r \ldots r$ \\
\hline$\leq 7 . \wedge$ & $\leq r_{.} 1$ & $r \cdot$. & Ir. & ov. $q$ & $\leq r .1$ & 101.1 & $r \cdot \cdot$ & TI. & Ir. & $r \leq 7 . \wedge$ & r. $\varepsilon$. & $1 \leq r \wedge$ & 1111 & voo & $r \ldots r$ \\
\hline ר & 00.1 & rV.l & iA.V & $\varepsilon \varepsilon, Y$ & 00.1 & Irیv.^ & r. & qr.. & IA.V & $\leqslant 70 . \wedge$ & $r \leq 10$ & 107r & $1.9 V$ & $v \cdot v$ & $r \ldots \varepsilon$ \\
\hline$r \cdot r$ & $09 . V$ & ro.^ & rT.q & $\varepsilon \cdot r$ & $09 . \mathrm{V}$ & $\mid V \leq \wedge . \wedge$ & ro.A & $1 \cdot \leqslant \wedge$. & rr.q & $\vee \cdot \cdot \wedge$ & ५१५. & INAT & 1111 & voo & r...o \\
\hline rA. $q$ & 7.9 & $r \cdot$. & $r \cdot .9$ & rq. & 7.9 & r.0 $0 \leqslant .1$ & $r \cdot$. & $1.1 r_{0} 0$ & $r \cdot .9$ & $1 \cdot \leq 1.7$ & mogo & מדזי & | & nto & $r \ldots r$ \\
\hline r..० & ר. & $\leqslant r . \varepsilon$ & r. & r.s & (ז. & $r \leqslant \curlyvee \wedge . \wedge$ & $\varepsilon r . \varepsilon$ & 1719. & r. & $\wedge \cdot 9 . \wedge$ & rat. & $r+1$ & $\mid r q 1$ & $\Lambda V_{0}$ & $r \cdots v$ \\
\hline rr.q & 77.7 & $\varepsilon \cdot .9$ & ro. & Tr. & 77.7 & $r \wedge \leqslant Y . r$ & $\varepsilon \cdot .9$ & $1 V \leq 0$. & ro. & $1.9 V . r$ & $\leq r 70$ & ror. & $1 \leq r r$ & 人9ז & $r \cdots \Lambda$ \\
\hline$r \cdot .1$ & $0 \wedge . \vee$ & rq. & $19 . r$ & $\varepsilon 1, r$ & $0 \wedge . \vee$ & rVדr.r & rq. & 110ะ. & $19 . r$ & $9.9 . r$ & $\leq v 1$. & r 107 & $19 \leq V$ & 1194 & $r \ldots q$ \\
\hline ro.9 & (ז.ק & $\varepsilon \varepsilon .9$ & $1 \wedge . \wedge$ & r. & (ז.ק & тr人т. & $\leqslant \leqslant .9$ & rMIT. & $1 \wedge . \wedge$ & $9 \vee \cdot \cdot$ & 0100 & rArq & 1179 & $11 \leq 1$ & $r \cdot 1 \cdot$ \\
\hline ro.r & $7 \leqslant .0$ & $\varepsilon r .7$ & $r \cdot \wedge$ & ro.T & $T \leqslant .0$ & r. & $\varepsilon r .7$ & $r \leq \varepsilon r$. & $r \cdot \wedge$ & $117 V . r$ & $07 .$. & rion & 1991 & MTK & $r \cdot 11$ \\
\hline rV.T & $\leqslant 9.9$ & $r \cdot$. & 19.9 & $0 . .1$ & $\leq 9.9$ & r. & $r \cdot$. & $|N| r .0$ & 19.9 & $M r . r$ & $7 . \leqslant 0$ & ETrT & r.r. & Mir & $r \cdot 1 r$ \\
\hline ז & 01.1 & $\leqslant 7 . \varepsilon$ & $\varepsilon .7$ & $\leqslant \wedge .9$ & 01.1 & ג.זוז & $\leqslant 7 . \leqslant$ & $r .1 r$. & $\varepsilon .7$ & $r \cdots . \wedge$ & $7 \leqslant 9$. & $r \leqslant V V$ & TIVT & 11990 & $r .1 T$ \\
\hline r^.r & $\leq 9$. & $\leq 0 . r$ & $r . v$ & 01. & $\leqslant 9$. & rrqv. & $\leq 0 . r$ & "r . & $r . v$ & roN. 7 & 7970 & rVqT & rorv & YI. & $r \cdot 1 \varepsilon$ \\
\hline$r V . \varepsilon$ & $\leqslant 9 . \wedge$ & $r \cdot \cdot$ & 19.1 & $0 . . r$ & $\leq 9 . \wedge$ & TTVV.V & $r \cdot \cdot$ & YYIS. & 19.1 & I & VrA. & 0177 & $r v \cdot r$ & Y)97 & $r .10$ \\
\hline or. 7 & $r \varepsilon .7$ & r. & Ir. & 70.5 & $r \varepsilon .7$ & $r v \cdot \tau . r$ & r.P.O & 1809. & Ir.1 & $q \leq V . r$ & VAYO & 7.77 & 0119 & $r \ldots o$ & $r .17$ \\
\hline ס.0.0 & $\wedge \wedge$ ^.^ & $0 \wedge 9.9$ & rq1.V & $\wedge \wedge . r$ & $\wedge \wedge \perp . \wedge$ & $r \wedge r \leq r . V$ & $0 \wedge 9.9$ & หчrqч.० & rq१.V & $I r \cdot \varepsilon V_{0} r$ & VYo.o & \&4YI. & एะ171 & $r \cdot \wedge \wedge l$ & الإجمالي \\
\hline$r v . \varepsilon$ & 01.9 & $r \varepsilon . V$ & IV.r & $\varepsilon \wedge$ & 01.9 & rro0.0 & $\Gamma \varepsilon . V$ & $10 \leqslant 7.9$ & IV.r & $v \cdot \Lambda \cdot v$ & \&YY & rVIA & $r \ldots q$ & Irrs & المتوسط \\
\hline
\end{tabular}

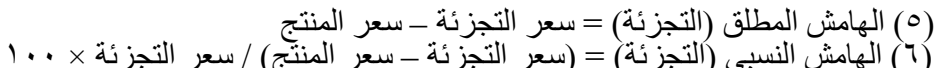

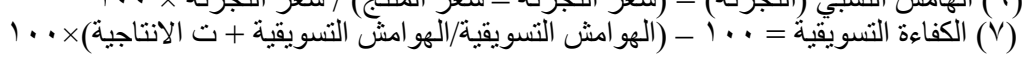

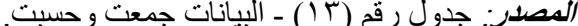

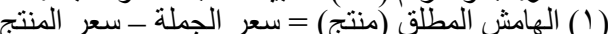

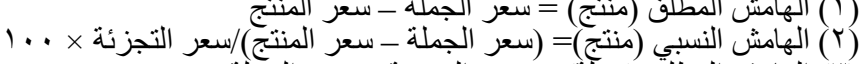

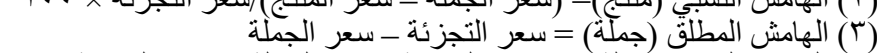

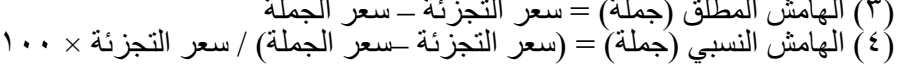




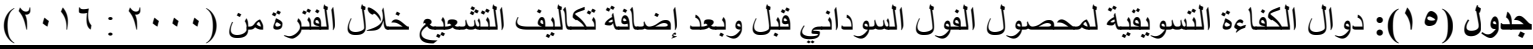

\begin{tabular}{|c|c|c|c|}
\hline $\mathbf{R}^{-2}$ & $\mathbf{F}$ & المعادلة & التختير \\
\hline 0.86 & $26.81^{* * *}$ & $\begin{aligned} \mathrm{E}= & 41.89+0.02 \mathrm{P}_{\mathrm{f}}-0.01 \mathrm{P}_{\mathrm{r}}+0.001 \mathrm{C}_{\mathrm{p}} \rightarrow(9) \\
& (19.24)^{* * *}(3.82)^{* * *}(-8.70)^{* * *}(0.09)^{* * *}\end{aligned}$ & قالكفاءة التسويقية \\
\hline 0.86 & $28.51^{* * *}$ & $\begin{aligned} \mathrm{E}= & \varepsilon q . \wedge \varepsilon+0.02 \mathrm{P}_{\mathrm{f}}-0.01 \mathrm{P}_{\mathrm{r}}+0.005 \mathrm{C}_{\mathrm{p}} \rightarrow(10) \\
& (12.82)^{* * *}(5.54)^{* * *}(-7.64)^{* * *}(0.99)^{* * *}\end{aligned}$ & بعد تكاليف التشويقية \\
\hline
\end{tabular}

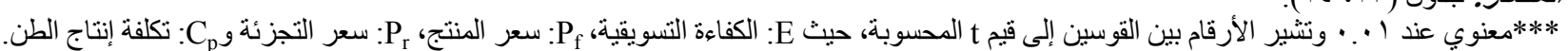

وتشير نتائج تقدير توزيع جنية المستهلك لمحصول البرتقال

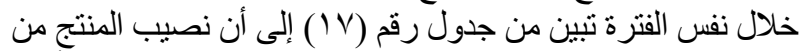

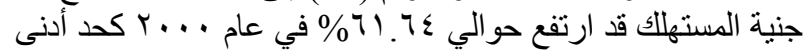

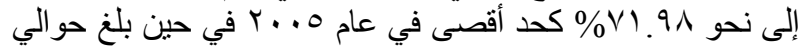

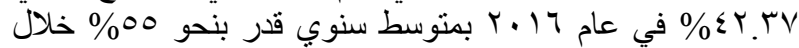
نفس الفترة. أما نصيب الوسطاء من جنية المستهلك فقد تذبذب حئ حيث

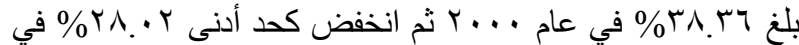

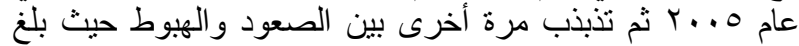

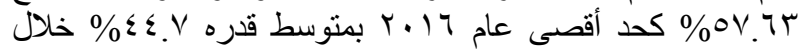

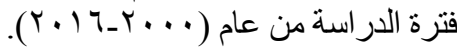

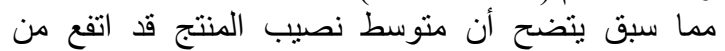

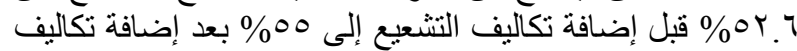

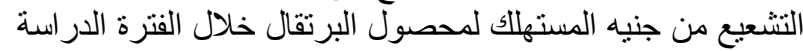

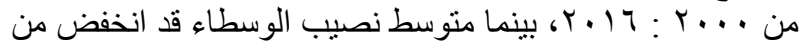

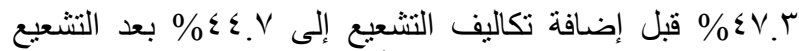

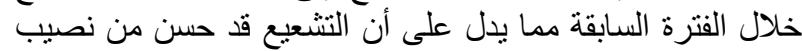
المنتج وحد من نصيب الوسطاء.

r- العوامل المؤثرة على الكفاءة التسويقية لمحصول البرتقال

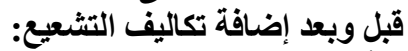

أوضحت النتائج في جدول رقالفم (IV) أن الكفاءة التسويقية

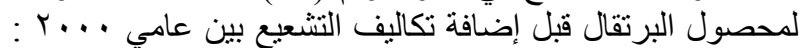

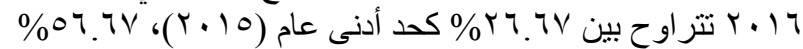

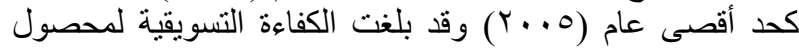

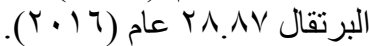
وتوضح دالة الكفاءة التسويقية لمحصول (بام البرتقال قبل إضافة

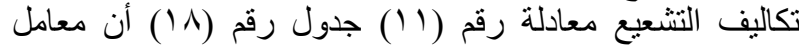

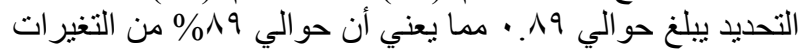

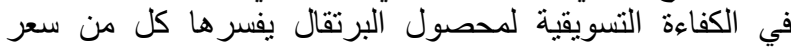

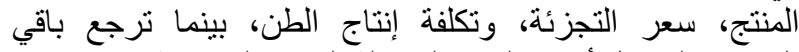

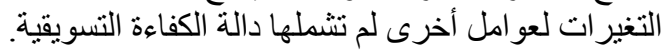

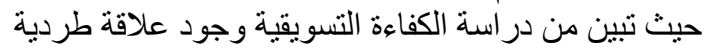
مؤكدة احصائياً بين كل من الكفاءة التسويقية كمتغير تنابع (E)

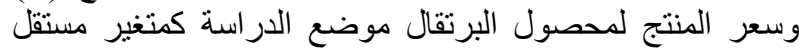

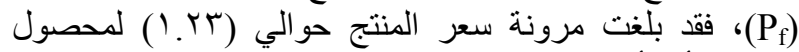

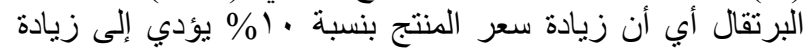

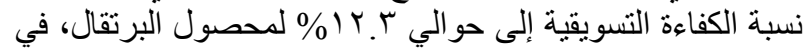

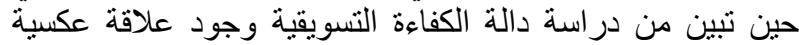
مؤكدة احصائياً بين كل من الكفاءة التسويقية كمتغير تابع (E)

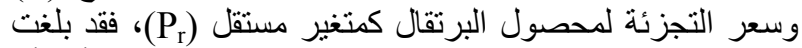

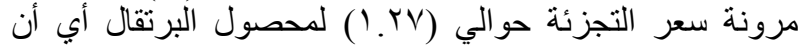

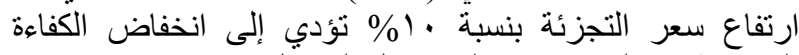

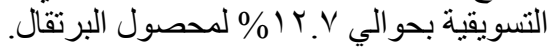

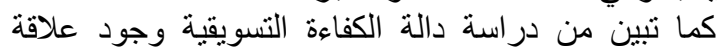

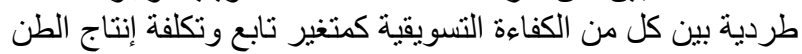

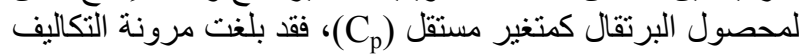
حوالي (1 1 . • ) لمحصول البرال كمغير مدتقل

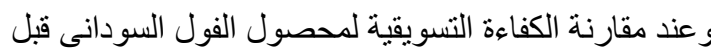
وبعد التشعيع تبين من النتائج السابقة ارتفاع الحد الأدنى للكفاءة الكناء

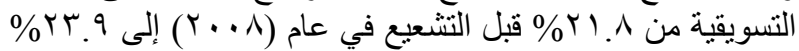

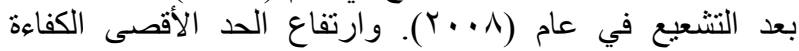

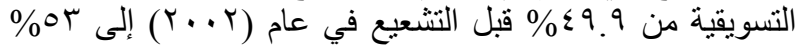

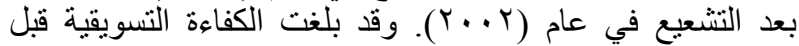

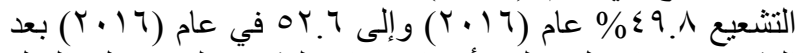

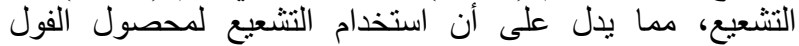

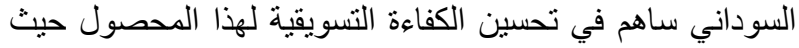

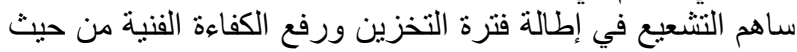

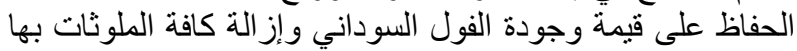

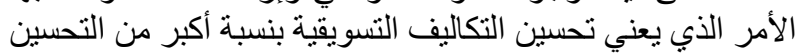

في التكاليف الإنتاجية.

سادساً: أثر استخدام التشعيع في تحسين الكفاءة التسويقية لمحصول البرتقال قبل وبعد إضافة تكاليف التشعيع في مصر.

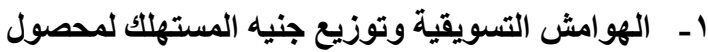
البرتقال قبل إضافة تكاليف التشعيع

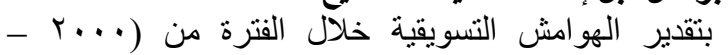

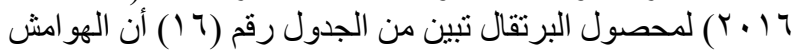

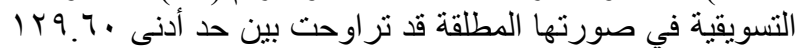

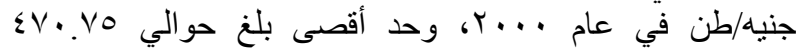

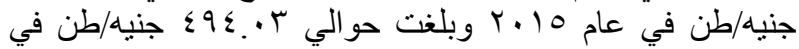

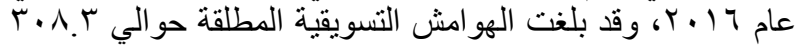
جنيه/طن كمتوسط لفترة الدراسة وذلك على على مستوى تاجر الجملة، A . . ا جنيه/طن على مستوى تاجر التجزئة.

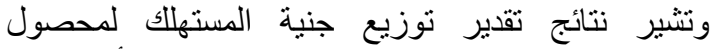

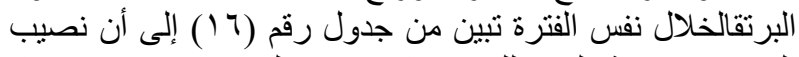

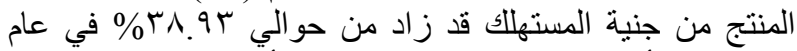

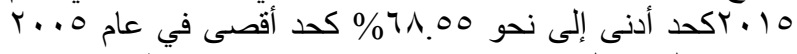

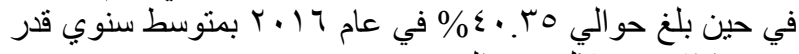

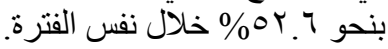

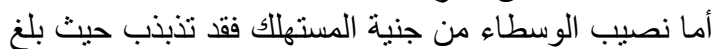

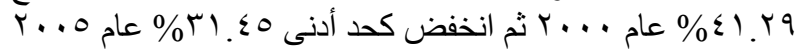

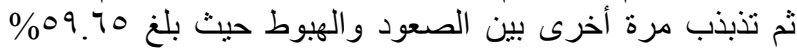

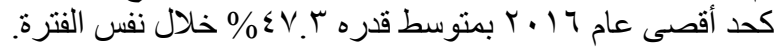

r- الهوامش التسويقية وتوزيع جنيه المستهلك لمحصول

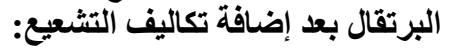

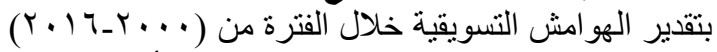

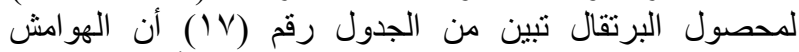

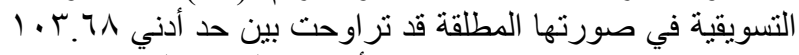

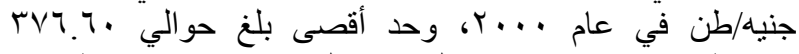

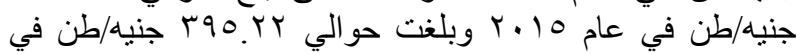

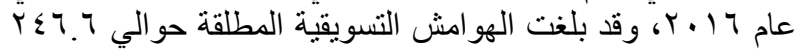

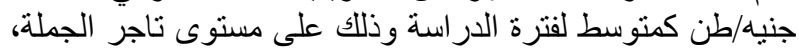

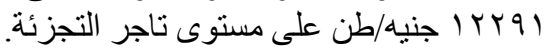




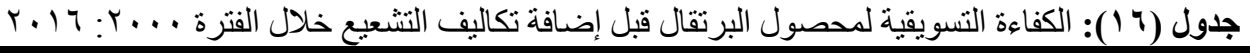

\begin{tabular}{|c|c|c|c|c|c|c|c|c|c|c|c|c|c|c|c|}
\hline \multirow{3}{*}{ التسويقية } & \multirow{3}{*}{$\begin{array}{c}\text { الوسطيبء } \\
\text { \% }\end{array}$} & \multirow{3}{*}{ 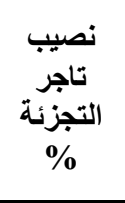 } & \multirow{3}{*}{ نصيب تاجر } & \multirow{3}{*}{$\begin{array}{l}\text { المنتجج } \\
\text { \% }\end{array}$} & \multicolumn{2}{|c|}{ الانتشار السعرى } & \multicolumn{4}{|c|}{ الهوامش التسويقية } & \multirow{3}{*}{ جنيه/طن التجنة } & \multirow{3}{*}{ جنيه/طن الجملة } & \multirow{3}{*}{ جنيه/طن المنتج } & \multirow{3}{*}{ 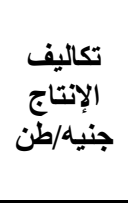 } & \multirow{3}{*}{ السنوات } \\
\hline & & & & & \multirow[b]{2}{*}{ النسبى } & \multirow[b]{2}{*}{ المطلق } & \multicolumn{2}{|c|}{ هامش التجزئـة } & \multicolumn{2}{|c|}{ هامش الجملة } & & & & & \\
\hline & & & & & & & النسبيى(") & الهاملق (") & النسبيى\%(") & الهامشق(') & & & & & \\
\hline$\leqslant 1.00$ & $\leqslant 1.49$ & r..71 & $1 \leq .71$ & $0 \wedge . \vee 1$ & $\leqslant 1.49$ & Tาะ.7 & $r 7.71$ & rro... & $1 \leq .71$ & $1 \times 9.7$. & גᄉr & $T \leqslant \Lambda$ & 011 & roq & $r \ldots$ \\
\hline rq.qr & $\leqslant V . \leqslant 0$ & $r \leqslant . r T$ & $14.1 \leq$ & or.00 & $\leqslant V . \leqslant 0$ & orl. & r & 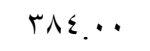 & $1 T .1 \varepsilon$ & $1 \leqslant v_{.}$. & 1119 & VTo & $0 \wedge \Lambda$ & ror & $r \ldots l$ \\
\hline$\leqslant 0.70$ & $\sum 1.7 \mathrm{~V}$ & $r v . \cdot q$ & $1 \leq .01$ & Tr.ron & $\sum 1.7 \mathrm{~V}$ & $\Sigma \| . \wedge$ & $r V . \cdot q$ & rчq... & $1 \leq .01$ & $1 \leq \leqslant . \wedge$. & 994 & $V Y \leq$ & ovq & $r \leq \Lambda$ & $r \ldots r$ \\
\hline$\leq 9 . r \wedge$ & rᄉ. $\cdot \Lambda$ & Yr.TI & $10 . \leqslant 1$ & 71.94 & rᄉ. & orq. & Yr.TI & M $\leq . .$. & 10.21 & rio... & $1 \pi \wedge 9$ & $1 \cdot 10$ & ᄉ५. & 017 & $r \ldots r$ \\
\hline$\leqslant \Lambda . O V$ & rᄉ..人 & $r$ r.OV & 10.19 & 71.10 & rᄉ..人o & ONT. & rT.OV & ros... & 10.19 & rrq.7. & $10 . r$ & $11 \leq 1$ & 911 & 001 & $r \ldots \varepsilon$ \\
\hline $07.7 \mathrm{~V}$ & $M . \leqslant 0$ & $\mid \leq . M$ & $\mid V .1 \leq$ & 71.00 & $M_{1} \leq 0$ & $\varepsilon r r_{.} \cdot$ & $1 \leq .4$ & $194 . .$. & $\mid V .1 \leq$ & $r r \cdot .$. & $1 r \leqslant r$ & 110. & $9 r$. & $00 Y$ & $r \ldots o$ \\
\hline$\varepsilon r . \vee T$ & $\leqslant r .0 \leqslant$ & $r q . \leqslant r$ & $1 \leq .1 T$ & $07 . \leqslant 7$ & $\varepsilon r .0 \leqslant$ & $V \leq 0 . \wedge$ & $r q . \leqslant r$ & $0 . \leqslant \ldots$ & $1 \leq .1 Y$ & $r \leqslant 1 . \wedge$. & IVIT & $1 r \cdot 9$ & 97V & $0 \wedge$. & $r \ldots r$ \\
\hline$\leq \Lambda .1 \leq$ & rq. r & $r \varepsilon . V$ & 10.19 & $7 \cdot . V \leqslant$ & rq.r & $V 71.7$ & $r \leq . \cdot V$ & $\leq T V_{.}$. & 10.19 & rqะ.7. & $19 \leq$. & $1 \leqslant V T$ & $11 \mathrm{VA}$ & $V \cdot v$ & $r \ldots r$ \\
\hline$\leqslant 7.91$ & $\varepsilon \cdot r \Lambda$ & $r_{0} \leqslant V$ & $1 \leq .91$ & 09.74 & $\varepsilon \cdot . r \Lambda$ & גז.זrA & $r_{0} \leqslant V$ & orr... & $1 \leq .91$ & $r \cdot v . \wedge$. & $r .70$ & 1049 & $|r T|$ & vrq & $r \ldots \Lambda$ \\
\hline$\leqslant 1 . \wedge 1$ & $\leqslant 0.0$. & r.AN & Tr.Tr & $0 \leqslant .0$. & $\leqslant 0.0$. & $11.0 . \mathrm{V}$ & M.AN & $V V \leq .71$ & IT.TY & ג•. וזr & $r \leqslant r$. & 1700 & ITKE & 190 & $r \ldots q$ \\
\hline$r v .9$ & $0 . . \leqslant \leqslant$ & rı.. 0 & Ir.rq & $\leqslant 9.07$ & $0 . . \leq \leqslant$ & $1 \leq \leqslant\} .7$ & rᄉ.. 0 & $\mid \cdot \wedge \Lambda . r 1$ & 1Y.rq & T T T & r人t. & IVVY & $I \leq 1 V$ & 10. & $r \cdot 1 \cdot$ \\
\hline$r \leq . r$. & or. $\leqslant v$ & $\leqslant 1 . \wedge \varepsilon$ & אד.711 & 纟7.0r & or. $\leqslant v$ & IVT.' & $\varepsilon 1 . \wedge \varepsilon$ & $1 r 0 \Lambda_{\varepsilon} \leqslant q$ & س.7. & TVV.T & $T Y \leqslant V$ & $\mid \wedge \wedge \Lambda$ & 1011 & 9.7 & $r .11$ \\
\hline rY..$\varepsilon$ & 00.99 & $\leq \leqslant .99$ & $11 \ldots$ & $\varepsilon \varepsilon . \cdot 1$ & 00.99 & $r \cdot \varepsilon \cdot .0$ & $\leq \leqslant .99$ & 1749.7. & $11 \ldots$ & $\leq \cdots .91$ & Tr & $r \ldots o$ & 17.5 & 974 & $r .1 T$ \\
\hline$r \cdot . r v$ & $0 \Lambda .+r$ & $\varepsilon V . O r$ & 1.0. & $\leqslant 1.91$ & $01 . \cdot r$ & $r r \leq \varepsilon .9$ & $\sum V . O T$ & $19 r \cdot . V \cdot$ & 1.0. & $\leq r \leq .19$ & $\varepsilon \cdot \varepsilon r$ & $Y|Y|$ & 1798 & 1.11 & $r .1 T$ \\
\hline$r \wedge . \wedge \varepsilon$ & 09.71 & $\leqslant 9.7$ & $1 \cdot .1$ & $\varepsilon \cdot r r$ & 09.71 & $r \tau \leq q . r$ & $\leq 9.7$. & $r r \cdot 1 . \wedge 1$ & $1 \cdot .1$ & $\leqslant \leqslant V . \leqslant V$ & $\varepsilon \leqslant \Psi q$ & TrTV & $1 \vee 9$. & $1 \cdot v \varepsilon$ & $r \cdot 1 \leq$ \\
\hline rV.TV & $71 . V$ & $01 . r \varepsilon$ & $9 . v r$ & $r \wedge .9 r$ & $71 . V$ & raor.v & $01 . \Gamma \varepsilon$ & $r \leqslant \wedge r . q r$ & $9 . V r$ & $\leq v \cdot v_{0}$ & $\varepsilon \wedge r V$ & rros & INAr & $11 \%$. & $r .10$ \\
\hline 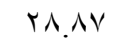 & 09.70 & $\leqslant 9.07$ & $1 \cdot .9$ & $\varepsilon \cdot$ r $_{0}$ & 09.70 & $r q r . . q$ & $\leqslant 9.07$ & $r \leq Y T . \wedge T$ & $1 . .9$ & $\leq q \leq .+r$ & $\varepsilon \wedge 9 \vee$ & $r \leqslant V$. & 1987 & 1117 & $r .17$ \\
\hline 711.01 & 1.0.vq & OAY.YO & YrY.OV & $\wedge q \varepsilon . Y$ & $1.0 v . q$ & rrrv^.q & OAY.ro & IVIrA.r & YrY.OV & Ors . .Tr & $\varepsilon r r \leqslant r$ & $r q r \cdot r$ & r.97 & I rory & الإجمالي \\
\hline$\varepsilon \cdot .99$ & $\varepsilon V . r$ & rE.Yo & 17.10 & or.t & $\leqslant V_{.} \varepsilon$ & 1417 & $r \leq . Y 0$ & $1 \cdots 1$ & 14.10 & $r \cdot \Lambda . r$ & $r 0 \leqslant q$ & $10 \leqslant 1$ & Irr & VTV & المتوسط \\
\hline
\end{tabular}
(0) الهامش وحسبت المطلق (التجزئة) = سعر التجزئة - سعر المنتج

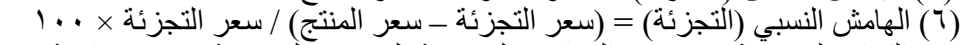

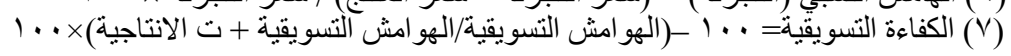

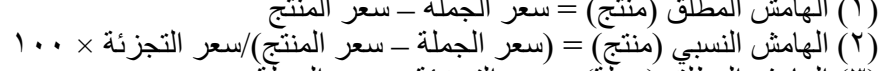

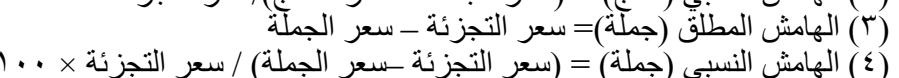




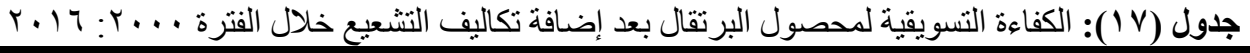

\begin{tabular}{|c|c|c|c|c|c|c|c|c|c|c|c|c|c|c|c|}
\hline \multirow{3}{*}{ التسويقية } & \multirow{3}{*}{$\begin{array}{c}\text { الوسطاء } \\
\text { \% }\end{array}$} & \multirow{3}{*}{$\begin{array}{c}\text { تصاجزيب } \\
\text { التجزئة } \\
\text { \% }\end{array}$} & \multirow{3}{*}{$\begin{array}{c}\text { نصيب } \\
\text { الجملة } \\
\text { \% }\end{array}$} & \multirow{3}{*}{ المنتج } & \multicolumn{2}{|c|}{ الانتثار السعرى } & \multicolumn{4}{|c|}{ الهوامش التسويقية } & \multirow{3}{*}{ جنيه/طن } & \multirow{3}{*}{ جنيه/طن } & \multirow{3}{*}{ جنيه/طن } & \multirow{3}{*}{ جنيه/طاليف } & \multirow{3}{*}{ السنوات } \\
\hline & & & & & \multirow[b]{2}{*}{ النسبى } & \multirow[b]{2}{*}{ المطلق } & \multicolumn{2}{|c|}{ هامش التجزئة } & \multicolumn{2}{|c|}{ هامش الجملة } & & & & & \\
\hline & & & & & & & 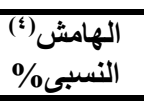 & الماملق الم") & النسبيى") & الهامشق المطق & & & & & \\
\hline Or.Ar & rᄉ.r & หฺ.7) & $11 . v \leq$ & $71.7 \varepsilon$ & rA.rT & rr..V & r..71 & rro... & $11 . v \varepsilon$ & $1 . r .71$ & גᄉr & $7 \leqslant \Lambda$ & $0 \leqslant \varepsilon$ & rvq & $r \ldots$ \\
\hline$\varepsilon \wedge .0 Y$ & $\leqslant \leqslant . \wedge r$ & $r \leqslant . r T$ & $1 \cdot .01$ & $00.1 \mathrm{~V}$ & $\varepsilon \varepsilon . \wedge r$ & 0.1 .7 & $r \leqslant . r T$ & rᄉะ... & $1 . .01$ & 118.7 & 1119 & VTO & $71 V$ & $\varepsilon V r$ & $r \ldots r$ \\
\hline $0 \leqslant .10$ & 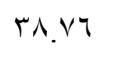 & $r V . \cdot q$ & $11.7 \mathrm{~V}$ & $T, Y \leq$ & 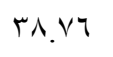 & $\Gamma \wedge \varepsilon . \wedge$ & $r V .9 q$ & rrq... & $11.7 \mathrm{~V}$ & $110 . \wedge \varepsilon$ & ११५ & $V Y \leq$ & 7.1 & $\leq 7 \wedge$ & $r \ldots r$ \\
\hline 07.71 & $r \leqslant .99$ & r.TI & Ir.rk & 70.1 & $r \leqslant .99$ & $\leqslant \wedge \uparrow$. & Tr.TI & TI... & ה.r. & IVT... & $1 \pi \wedge 9$ & 1. vo & 9.4 & דזד & $r \ldots r$ \\
\hline $00.0 r$ & ro.s. & rT.OV & MTr & $T \leq, Y$. & ro.A. & orv. & rr.ov & ros... & Tr.rT & 1ג. & $10 . r$ & $11 \leqslant 1$ & $97 \varepsilon$ & TVI & $r \ldots \varepsilon$ \\
\hline$T \leq .1 T$ & $r \wedge . r$ & $1 \leq . r \mid$ & $|r . v|$ & $\vee 1.91$ & $r \wedge . \cdot r$ & rV.. & $1 \leq . r \mid$ & $194 . .$. & $|r . v|$ & $\mid \wedge \varepsilon .$. & $1 T \leqslant r$ & 110. & 977 & TVY & $r \ldots o$ \\
\hline $0 . .1$. & $\varepsilon \cdot v)$ & $r q . \leqslant r$ & $11 . r_{9}$ & 09.19 & $\varepsilon \cdot V)$ & $79 \vee . \varepsilon$ & $r q . \leqslant r$ & $0 . \varepsilon .$. & 11.49 & $194 . \leqslant \varepsilon$ & IVIT & $1 Y \cdot 9$ & 1.17 & $v \ldots$ & $r \ldots r$ \\
\hline $0 \leqslant .7$ & T., & $r \varepsilon . V$ & $1 T_{10}$ & תז. & T. TY & $V \cdot r . V$ & $r \varepsilon . \cdot V$ & $\leq 7 V_{.}$. & 15.10 & rro.TA & $19 \varepsilon$. & $1 \leqslant V T$ & ITrV & ATV & $r \ldots v$ \\
\hline or.7o & $r v_{.} \varepsilon \cdot$ & $r_{0} \leqslant V$ & $11.9 r$ & Tr.T. & rv. $\varepsilon$. & VVY.Y & $r_{0} \leqslant V V$ & ort... & $11.9 r$ & $r \leq Y, r \leq$ & r. 70 & 1049 & Irar & 109 & $r \ldots \Lambda$ \\
\hline$\leq\rceil . \wedge$. & $\varepsilon r . \vee \wedge$ & $r . \wedge \Lambda$ & $1 \cdot .9$. & OV.YY & $\varepsilon r . \vee \wedge$ & $1 .+9.0$ & T.A1 & $V V \leq .71$ & 1.9. & 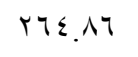 & $r \leqslant r$. & 1700 & $|r q|$ & 910 & $r \ldots q$ \\
\hline$\varepsilon 1 . \leqslant \mu$ & $\leqslant V .97$ & $r \wedge . .0$ & 9.91 & or. $\cdot \varepsilon$ & $\sum V .97$ & $|r v| . v$ & rı.. 0 & $|\cdot \lambda \Lambda . r|$ & 9.91 & rAr. $\varepsilon q$ & rAT. & IVVT & $1 \leq \wedge \Lambda$ & q४. & $r \cdot 1 \cdot$ \\
\hline rл.r. & 01.10 & $\varepsilon 1 . \wedge \varepsilon$ & 9.4 & $\leqslant \wedge . \wedge 0$ & 01.10 & 177.7 & $\S . \wedge \varepsilon$ & $1 T 01 . \leqslant 9$ & 9.4 & $r \cdot r .11$ & $T r \leqslant V$ & $|\wedge \wedge|$ & 1017 & 1.YT & $r .11$ \\
\hline ro.ov & or.va & $\leqslant \leqslant .99$ & $\wedge . \wedge$ & $\leq 7, r)$ & or.va & $197 \cdot . r$ & $\leqslant \leqslant .99$ & 1749.7. & $\wedge . \wedge$ & $r r \cdot V r$ & ع & $r \ldots o$ & $171 \varepsilon$ & $1.1 r$ & $r \cdot 1 r$ \\
\hline Q & 00.94 & $\sum V . O r$ & $\Lambda . \varepsilon$. & $\leq \varepsilon . \wedge$ & 00.94 & YrT.. & $\varepsilon V . O r$ & $19 r \cdot . V \cdot$ & $\wedge . \varepsilon$. & סף. & $\varepsilon \cdot \leq r$ & YIY & IVAT & I & $r \cdot 1 r$ \\
\hline M. II & OV.TT & $\leqslant 9.7$ & $\Lambda .7$ & $\varepsilon r . r \leqslant$ & $0 V .77$ & ro09.1 & $\leqslant 9.7$ & $r+1 . \wedge 1$ & A. .7 & rov.91 & $\varepsilon \leqslant r q$ & TrTV & 1189 & $119 \leq$ & $r \cdot 1 \leq$ \\
\hline$r \cdot . \Sigma l$ & 09.14 & $01 . r \varepsilon$ & $\vee . \vee q$ & $\varepsilon \cdot . \wedge$ & 09.14 & r^०9.0 & $01 . r \leq$ & $r \leq \wedge r . q r$ & V.Vq & rVT.T. & 纟ภrv & Tros & $19 V V$ & iro. & $r \cdot 10$ \\
\hline זד. וץ & OV.T & $\{9.07$ & $\Lambda . \cdot \vee$ & $\varepsilon r . r V$ & ov.7r & rAYr.l & $\leqslant 9.07$ & $r \leqslant Y T . \wedge T$ & ^. $\cdot V$ & rq०. r & $\varepsilon \wedge 9 \vee$ & $r \leqslant V$. & r. vo & $1 T \cdot 7$ & $r .17$ \\
\hline Vマ^.7 & V4.1 & OAY.YO & $\mid \vee \wedge . \wedge \leq$ & $9 r \wedge . q$ & V.1. & $r \mid r r \cdot V$ & OAY.YO & $r \cdot \wedge q \leq q . r$ & $\mid \vee \wedge . \wedge \leq$ & $\leqslant 194.0$ & $\varepsilon r r \leqslant r$ & ห५q ч & $r r+1$. & $1 \leqslant 074$ & الإجمالي \\
\hline$\varepsilon 0 . \wedge$ & $\varepsilon \varepsilon . V$ & $r \varepsilon . Y_{0}$ & $1.0 r$ & 00 & 8.7 & Iros.V & $r \leqslant . r_{0}$ & $|r+q|$ & $1.0 r$ & $r \leq 4.7$ & $r 0 \leq q$ & $1 \varepsilon .9$ & $1 \times 90$ & Nov & المتوسط \\
\hline
\end{tabular}

(0) الهامش المطلق (التجزئة) = سعر التجزئة - سعر المنتج

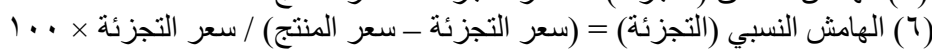

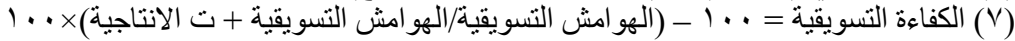

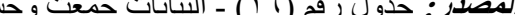

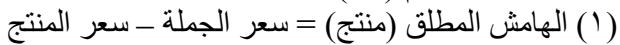

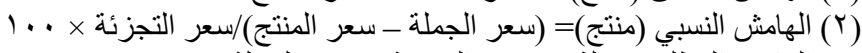

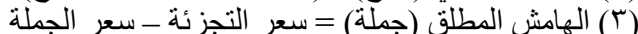

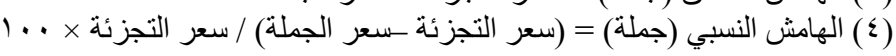




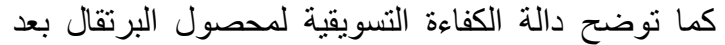

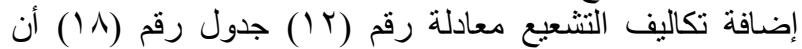

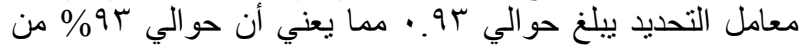

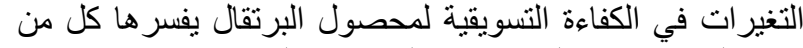

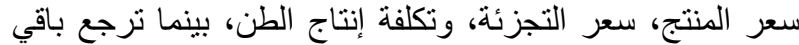

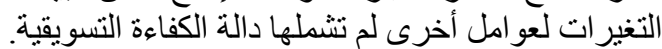

أوضحت النتائج في جدول رقم (IV) أن الكفاءة التسويقية

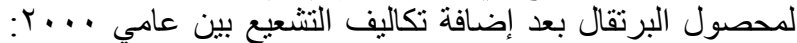

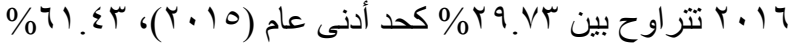

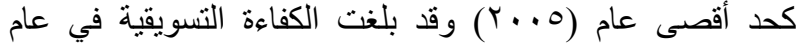
$. \% r \cdot . \wedge 9(Y \cdot 17)$

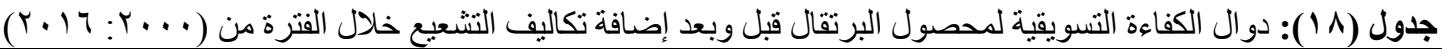

\begin{tabular}{|c|c|c|c|}
\hline $\mathbf{R}^{-2}$ & $\mathbf{F}$ & المعادلة & 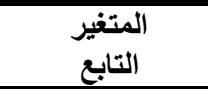 \\
\hline$\because \wedge 9$ & $35.9^{* * *}$ & $\begin{aligned} \mathrm{E}= & 41.27+0.04 \mathrm{P}_{\mathrm{f}}-0.02 \mathrm{P}_{\mathrm{r}}+0.01 \mathrm{C}_{\mathrm{p} \rightarrow(11)} \rightarrow(11) \\
& (12.01)^{* * *}(3.84)^{* * * *}(-6.0)^{* * *}(-1.10)^{* * *}\end{aligned}$ & تكاليف التشاعة التسويقية \\
\hline 0.93 & $57.9^{* * *}$ & $\begin{aligned} \mathrm{E}=0 \mathrm{~V} . & \tau \mathrm{r}+0.003 \mathrm{P}_{\mathrm{f}}-0.011 \mathrm{P}_{\mathrm{r}}+0.001 \mathrm{C}_{\mathrm{p}} \rightarrow(12) \\
& (13.88)^{* * *}(0.21)^{* * *}(-3.66)^{* * *}(1.65)^{* * * *}\end{aligned}$ & تكاليف التفاعة الضافويقية \\
\hline
\end{tabular}

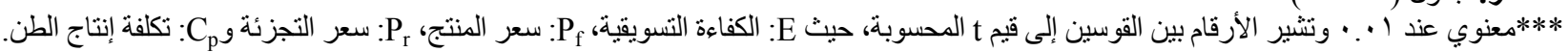

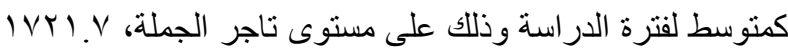
جنيه/طن على مستوى تاجر التجزئة.

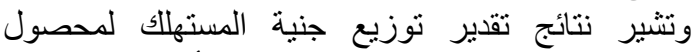

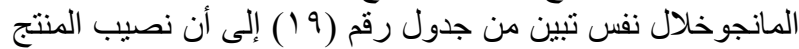

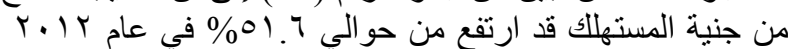

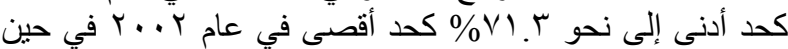

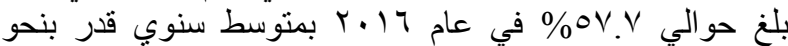

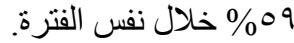

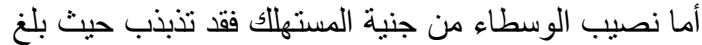

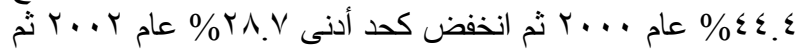

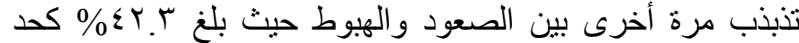

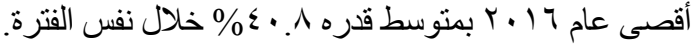

r- الهوامش التسويقية وتوزيع جنيه المستهلك لمحصول الماتجو بعد إضافة تكاليف التشعيع:

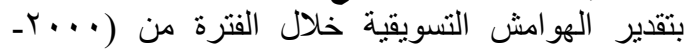

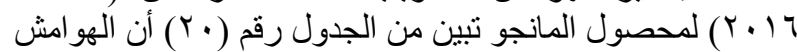

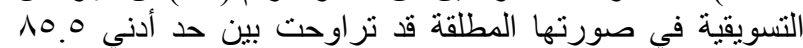

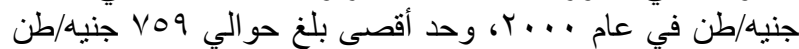

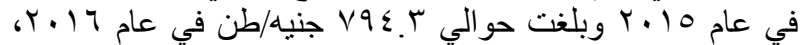

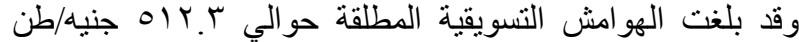

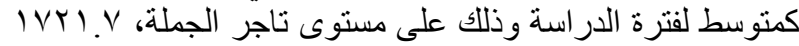
جنيه/طن على مستوى تاجر التجزئة.

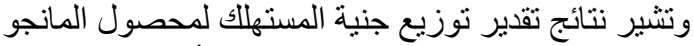

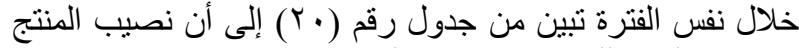

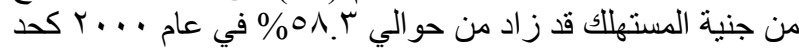

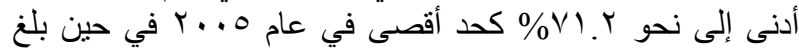

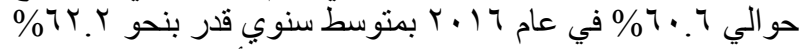

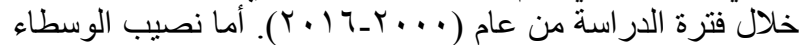

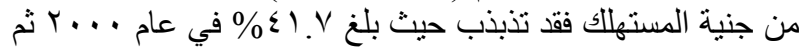

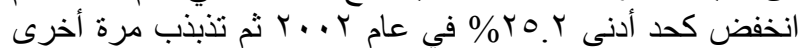

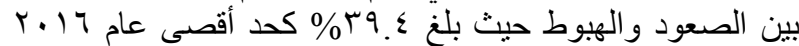

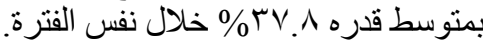

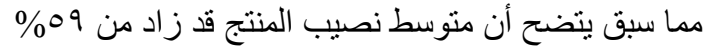

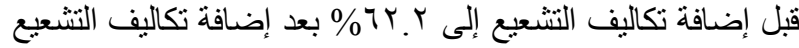

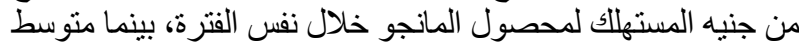

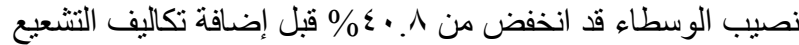

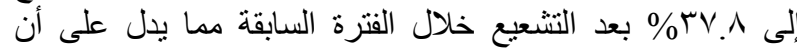
التشعيع قد حسن من نصيب المنتج وحد من نصيب الوسطاء.
حيث تبين من در اسة الكفاءة التسويقية وجود علاقة طردية

مؤكدة احصائياً بين كل من الكفاعة التسويقية كمتغير نابع (E)

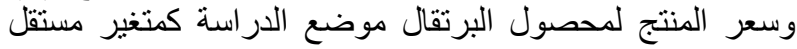

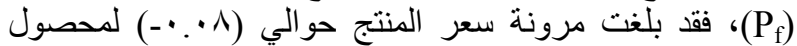

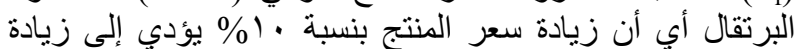

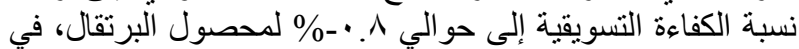

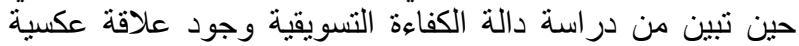
مؤكدة احصائياً بين كل من الكفاءة التسويقية كمتغير تنابع (E)

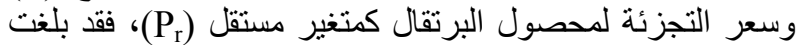

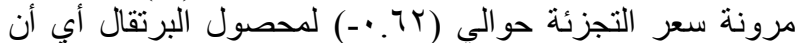

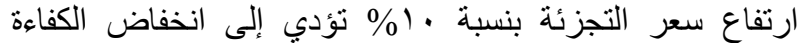

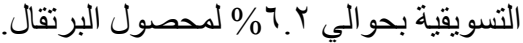

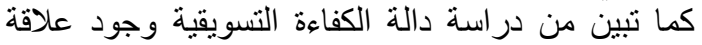
طردية بين كل من الكفاءة التسويقية كمتغير تنابع وتكلفة إنتاج الطن

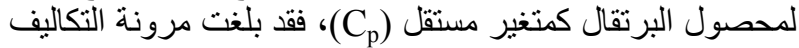

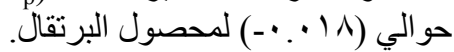

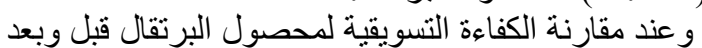
تكاليف التشعيع تبين من النتائج السابقة ارتفاع الحفة الحد الأدنى للكفاءة

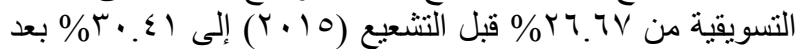

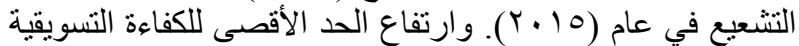

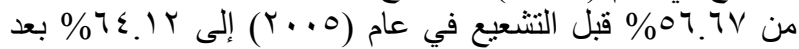

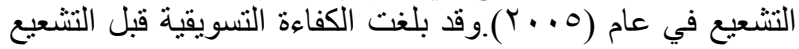

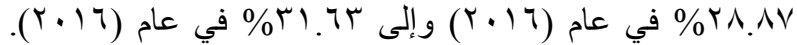

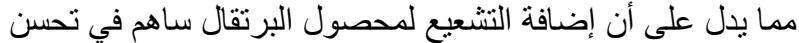
الكفاءة التسويقية لهذا المحصول حيث إنه ساهم التشعيع في إطالة فترة

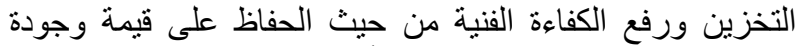
البرتقال و إز الة كافة الملوثات بها الأمر الذية من الذي يعني تحسين التكاليف التسويقية بنسبة أكبر من التحسين في التكاليف الانتاجية.

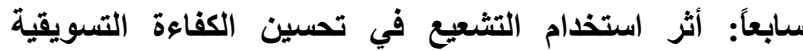
لمحصول المانجوقبل وبعد إضافة تكاليف التشعيع في مصر.

1- الهوامش التسويقية وتوزيع جنيه المستهلك لمحصول المانجو قبل إضافة تكاليف التشعيع

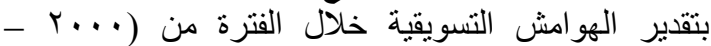

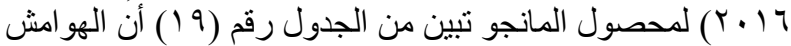

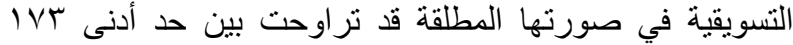

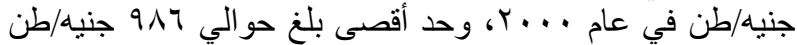

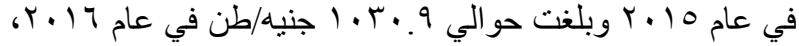
وقد بلغت الهوامش التسويقية المطلقة حوالي TV TV جنيه/طن 


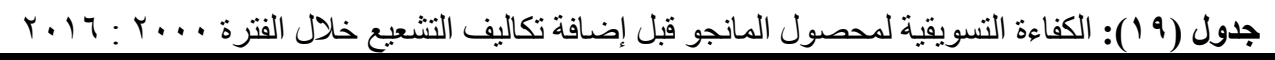

\begin{tabular}{|c|c|c|c|c|c|c|c|c|c|c|c|c|c|c|c|}
\hline \multirow{3}{*}{$\begin{array}{c}\text { التسويقية } \\
\text { \% }\end{array}$} & \multirow{3}{*}{$\begin{array}{c}\text { الوسطاء } \\
\text { \% }\end{array}$} & \multirow{3}{*}{$\begin{array}{c}\text { نتجيب } \\
\text { التجزئة } \\
\text { \% }\end{array}$} & \multirow{3}{*}{ نصيب تاجر } & \multirow{3}{*}{ المنيب } & \multicolumn{2}{|c|}{ الانتشار السعرى } & \multicolumn{4}{|c|}{ الهوامش التسويقية } & \multirow{3}{*}{ " التجزئة } & \multirow{3}{*}{ جنيه/طنة } & \multirow{3}{*}{ جنيه/طن المنتج } & \multirow{3}{*}{ تكاليف } & \multirow{3}{*}{ السنوات } \\
\hline & & & & & \multirow[b]{2}{*}{ النسبى } & \multirow[b]{2}{*}{ المطلق } & \multicolumn{2}{|c|}{ هامش التجزئة } & \multicolumn{2}{|c|}{ هامش الجملة } & & & & & \\
\hline & & & & & & & النسامشي(") & المطامش ("الق & النسامشي(") & المطلق المامش") & & & & & \\
\hline$\leq 7 . \vee$ & $\varepsilon \varepsilon . \varepsilon$ & rq. & 0.0 & 00.7 & $\leqslant \leqslant . \leqslant$ & $1 \leq \cdots$ & rq. & IKr.. & 0.0 & IVT. & Mlo. & 19rr & 1vo. & IrTo & $r \ldots$ \\
\hline or. s & rv.q & r.T.T & $10 . r$ & Tr.1 & rv. $q$ & $\| \mu \cdot$ & r.T.T & TVT. & $10 . r$ & sov. & rqA. & $r T \cdot V$ & 110. & 1490 & $r \ldots l$ \\
\hline ع. & Y^.V & 17.8 & $\mid Y .1$ & $v i r$ & YA.V & $\Delta r v$. & 17.8 & $\varepsilon \Lambda \cdot$. & IT.1 & $r \leq v$. & rAVV & rrqV & r.o. & $1 \leqslant r 0$ & $r \ldots r$ \\
\hline $0 \leqslant . Y$ & r. . V & 19.1 & $1 V .7$ & ז.זיד & r.. & $M I V$. & 19.1 & Tा. & $1 V .7$ & oגז. & VוTr & TTAT & 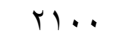 & $1 \leqslant V$. & $r \ldots r$ \\
\hline$\leqslant 7.0$ & $\leqslant \leqslant .7$ & $r V . r$ & IV.r & $00 . \leqslant$ & $\leqslant \leqslant .7$ & IVqY. & $r V . r$ & $1.9 V$. & IV.r & 790. & $\varepsilon .1 V$ & rqr. & TrYO & 1001 & $r \ldots \varepsilon$ \\
\hline 09.7 & r. & ro.1 & $v$. & $7 V .9$ & r.t. & 1500. & ro.1 & 1.01. & $v_{.} \cdot$ & rqv. & $\sum r 10$ & MIOV & үᄉт. & $r \ldots r$ & $r \ldots o$ \\
\hline 07.1 & ro.s & $r r$. & 1r. & $7 \leqslant .7$ & ro.s & lork.r & $r r_{.} \cdot$ & 907. & $1 \pi . \varepsilon$ & ONYT & sto. & צq זr & rAIr & 1971 & $r \ldots r$ \\
\hline ov. & $r \leqslant .0$ & $r \cdot . q$ & IT.V & 10.0 & $r \leqslant .0$ & 10NE. & $r \cdot . q$ & $90 \mathrm{~V}$. & IT.V & $T Y V . r$ & $\varepsilon 0 \wedge 1$ & ודוT & $r \ldots \varepsilon$ & $r) \cdot r$ & $r \ldots v$ \\
\hline ov. 7 & $r \leq$. & $r \cdot r$ & $1 r .9$ & 77. & $r \varepsilon$. & $17 \leqslant 9.0$ & $r \cdot r$ & $9 \vee \vee .0$ & $1 \% .9$ & TVY. & $\leqslant \wedge \leqslant 7$ & r人тА & T197 & TrTV & $r \ldots \lambda$ \\
\hline$\leq 0 . r$ & $\leqslant 0.1$ & $r \leqslant . r$ & 11.0 & $0 \leqslant . Y$ & $\varepsilon 0 . \wedge$ & ห人т). 9 & $r \leqslant . r$ & $r) \leq 0$. & 11.0 & V17.9 & tro. & $\leqslant 1.0$ & Aגו & TrVt & $r \ldots q$ \\
\hline$\leq 7 . r$ & $\leqslant \leqslant .9$ & rT. & $11 . \mathrm{V}$ & 00.1 & $\leqslant \leqslant .9$ & rq।V.० & rT. & r100.1 & $11 . \mathrm{V}$ & $\vee 7 l . \wedge$ & $7 \leq 91$ & $\varepsilon r \leqslant r$ & ron. & ro. & $r \cdot 1$. \\
\hline$\varepsilon \varepsilon, r$ & $\leqslant \uparrow . \wedge$ & ro.s & $11 . \varepsilon$ & or. r & $\varepsilon\urcorner . \wedge$ & TrYI.l & ro.s & $r_{0} \mid \leq 0$ & $11 . \varepsilon$ & 1.7 .7 & $V \cdot q \varepsilon$ & 纟ov9 & TVYT & YT & $r+11$ \\
\hline$\varepsilon r . V$ & $\varepsilon \wedge . \varepsilon$ & $r v . \varepsilon$ & 11.1 & 01.7 & $\varepsilon \wedge . \varepsilon$ & $r V Y \leq . V$ & $r v . \varepsilon$ & rAvr.r & 11.1 & 101.0 & Vฯ1 & $\{\wedge 17$ & ए970 & rVVo & $r \cdot I r$ \\
\hline$\varepsilon r . \wedge$ & $\sum V . r$ & ro. 9 & $11 . \varepsilon$ & or.y & $\varepsilon V . r$ & TVYA.r & ro.9 & rATr. & $11 . \varepsilon$ & 197. & VA^O & 0.04 & $\leqslant 10 \mathrm{~V}$ & rq1. & $r .1 T$ \\
\hline$\leq 0.1$ & $\leqslant 0 . \varepsilon$ & ס ס ז & 11.1 & $0 \leqslant .7$ & $\leq 0 . \varepsilon$ & r. & ס שי & หัтА. & 11.1 & $9 \leq 1.4$ & 1901 & orq. & $\leq r \leq q$ & $r \cdot \leq \varepsilon$ & $r \cdot 1 \leq$ \\
\hline$\left.\leqslant v_{.}\right)$ & $\varepsilon \varepsilon$. & T.1. & Ir. & 07. & $\varepsilon \varepsilon$. & rora. & $r . \wedge$ & roNt. & $M . r$ & 9人.. & 111. & $00 \mathrm{rV}$ & $\leq 0 \leq 1$ & TIVq & $r \cdot 10$ \\
\hline$\leqslant 1.9$ & $\varepsilon r . r$ & rq.V & $1 Y .7$ & ov. . & $\varepsilon r . r$ & $r \leq 71 . q$ & rq.V & $r \leq r \Lambda$. & $1 Y .7$ & $1 \cdot r \cdot .9$ & $A r \cdot r$ & OVTE & EVTr & זוזr & $r .17$ \\
\hline 1०9.1 & 79T. & $\leq \wedge \leq .1$ & $r .9 .0$ & $1 \cdots 7.1$ & 994.4 & एя११५. & $\leq \wedge \leq .1$ & rqrqq.1 & 4.9 .0 & $11 \leq r \leq . \wedge$ & 90.19 & 70V0 & $0 \leqslant r r r$ & $r \Lambda \cdot r r$ & الإجمالي \\
\hline $0 . .0$ & $\varepsilon \cdot . \vee \wedge$ & $r \wedge .0$ & Ir & 09 & $\varepsilon \cdot . \vee \wedge$ & $r r r \leqslant . q$ & $r \wedge .0$ & IVYI.V & ir & TVY & $\Delta \Delta \wedge q$ & 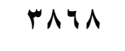 & 4197 & rrrv & المتوسط \\
\hline
\end{tabular}

(0) (الهامش المطلت (التجزئة) = سعر التجزئة - سعر المنتج

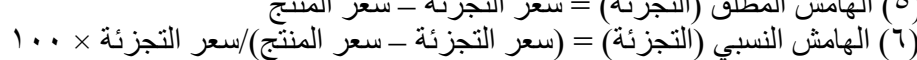

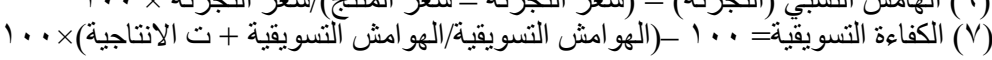

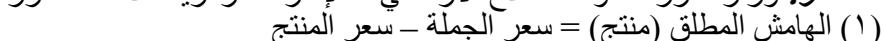

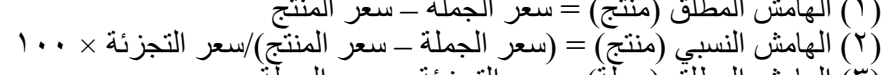

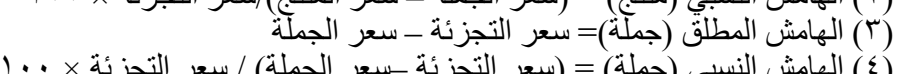




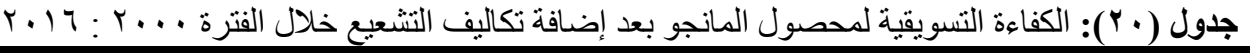

\begin{tabular}{|c|c|c|c|c|c|c|c|c|c|c|c|c|c|c|c|}
\hline \multirow{3}{*}{ التسويقية } & \multirow{3}{*}{ الوسطيء } & \multirow{3}{*}{$\begin{array}{c}\text { نتاجزيب } \\
\text { التجزئة } \\
\text { \% }\end{array}$} & \multirow{3}{*}{ نصيب تاجر } & \multirow{3}{*}{ المنتج } & \multicolumn{2}{|c|}{ الانتثار السعرى } & \multicolumn{4}{|c|}{ الهوامش التسويقية } & \multirow{3}{*}{ جنيه/طن التجنة } & \multirow{3}{*}{ جنيه/طن الجملة } & \multirow{3}{*}{ جنيه/طن المنتج } & \multirow{3}{*}{ 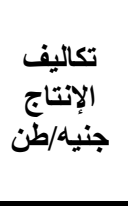 } & \multirow{3}{*}{ السنوات } \\
\hline & & & & & \multirow[b]{2}{*}{ النسبى } & \multirow[b]{2}{*}{ المطلق } & \multicolumn{2}{|c|}{ هامش التجزئة } & \multicolumn{2}{|c|}{ هامش الجملة } & & & & & \\
\hline & & & & & & & 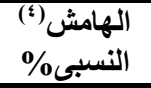 & الهامشق (") & النسبيى\%(") & المطامشق (') & & & & & \\
\hline $01 . Y$ & «. . & rq. & r.v & OA.r & $\varepsilon 1 . V$ & $M M .0$ & rq. & ITrV. & r.. & 10.0 & Mlo. & I9r & INH人 & irvo & $r \ldots$ \\
\hline$O \Lambda . Y$ & $r \leqslant . \wedge$ & TY.T & IT.Y & $70 . Y$ & $r \leqslant . \wedge$ & $1 . r v .0$ & rY.T & TVT. & Ir.r & rus.0 & rqA. & $r r \cdot v$ & $19 \leqslant r$ & $1 \leq \leqslant 0$ & $r \ldots l$ \\
\hline 71.7 & ro.r & 17.8 & 1.0 & $V \leqslant . \wedge$ & ro.r & $V Y \leq .0$ & 17.8 & $\leqslant \wedge \cdot$. & 1.0 & $r \leq \leq .0$ & rNVV & rrqV & rlor & 1010 & $r \ldots r$ \\
\hline $09 . r$ & ס.זr & 19.1 & $1 \leqslant . \varepsilon$ & 77.0 & ס. & 1114. & 19.1 & דะ. & $1 \leqslant . \varepsilon$ & $\varepsilon \vee \wedge$. & & rצו & Tr.O & ITr. & $r \ldots r$ \\
\hline $0 . . \varepsilon$ & $\S 1 . \wedge$ & TV.r & $1 \leqslant 0$ & $O \wedge . Y$ & $\leq 1 . \wedge$ & $171 \cdot .1$ & rV.r & $1.9 V$. & $1 \leq 0$ & 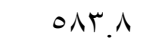 & $\varepsilon .1 V$ & rqr. & דומשr & $1 V \cdot \Lambda$ & $r \ldots \varepsilon$ \\
\hline$T \leqslant$. & $r \wedge . \wedge$ & ro.1 & r.v & $V I . Y$ & $r \wedge . \wedge$ & $M T K$. & ro. 1 & 1.01. & r.v & $10 \leqslant$. & $\leqslant r 10$ & MIOV & $r$ & rlor & $r \ldots o$ \\
\hline$T \cdot . r$ & r.l & $r r_{.} \cdot$ & $1 \cdot r$ & $7 \vee .9$ & r.. & IrqV.^ & $r r_{.} \cdot$ & 907. & $1 \cdot . r$ & $\leqslant \leqslant 1 . \wedge$ & $\leqslant$ so. & rrqs & r90 & YIA & $r \ldots r$ \\
\hline 71.1 & r. & $r \cdot . q$ & $1 \cdot . \varepsilon$ & $7 \wedge . V$ & r. & $1 \leq r \leq$. & $r \cdot . q$ & $90 \mathrm{~V}$. & $1 \cdot . \varepsilon$ & $\varepsilon V V$. & $\leqslant O \wedge \Lambda$ & וזדי & Mlos & rrot & $r \ldots v$ \\
\hline 71.7 & $r \cdot . v$ & $r \cdot r$ & $1 \cdot .7$ & $79 . r$ & $r \cdot . v$ & $1 \leqslant \wedge 9 . \wedge$ & $r \cdot r$ & $9 \vee \vee .0$ & $1 \cdot .7$ & $01 Y . T$ & $\leqslant \Lambda \leqslant 7$ & ґАт^ & Trot & TrAV & $r \ldots \Lambda$ \\
\hline$\leqslant \wedge . \varepsilon$ & $\varepsilon r .1$ & $r \leqslant . r$ & $\wedge . \wedge$ & 07.9 & $\varepsilon r .1$ & r.9Y.0 & $r \varepsilon . r$ & $r) \leq 0$. & $\wedge . \wedge$ & $0 \leqslant V .0$ & tro. & $\leqslant 1.0$ & roov & rort & $r \ldots q$ \\
\hline$\varepsilon q . r$ & $\left.\leq r_{.}\right)$ & Tr. & 9. & ov. 9 & $\varepsilon r .1$ & YVTA.O & Tr.t & r100.1 & 9. & 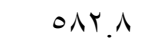 & $7 \leqslant 91$ & $\leq r \leq r$ & rvoq & r707 & $r \cdot 1$. \\
\hline$\left.\leq V_{.}\right)$ & $\varepsilon \varepsilon . Y$ & ro.s & ^. V & $00 . \wedge$ & $\varepsilon \varepsilon . r$ & ס.זוT & ro.s & rol $\leqslant 0$ & $\wedge . \vee$ & $71 \wedge$. & $V \cdot 9 \varepsilon$ & sovq & एवтा & rVq) & $r .11$ \\
\hline$\varepsilon 0 . r$ & $\leqslant 0.9$ & rv. & 1.0 & $0 \leq .1$ & $\leq 0.9$ & rort.o & $r v . \varepsilon$ & rNVT.r & 1.0 & Tor.r & $\vee \vee \wedge 9$ & $\sum \Lambda 17$ & ร17 & rqYo & $r .1 T$ \\
\hline$\leqslant 7.0$ & $\varepsilon \varepsilon .7$ & ro. 9 & ^. $\vee$ & 00.5 & $\varepsilon \varepsilon .7$ & ror. o & ro.9 & rATr. & ^. $\vee$ & $7 \wedge \wedge .0$ & VA^० & 0.04 & 纟หา & $r \cdot \tau$. & $r .1 T$ \\
\hline$\leqslant 1.0$ & $\leq Y_{.} T$ & o & 9.1 & ov. $\varepsilon$ & $\varepsilon r . T$ & ^. & rr.o & r7rı. & 9.1 & VYT.A & V৭०ᄉ & orq. & 5077 & M19 & $r \cdot 1 \leq$ \\
\hline$\leq 9.9$ & $\varepsilon 1 . r$ & $M .1$ & $9 . \varepsilon$ & $0 \wedge . \wedge$ & $\varepsilon 1 . r$ & $r r \leqslant r$. & 1.1 & ront. & $9 . \varepsilon$ & voq." & $\wedge 1$. & OOKV & $\leqslant \vee \neg \Lambda$ & Truq & $r .10$ \\
\hline $01 . V$ & $r q . \varepsilon$ & rq. . & 9.7 & $7 . .7$ & rq.ร & TrTY & rq.V & $r \leqslant r \Lambda$. & 9.7 & Vqะ.r & $\lambda r \cdot r$ & OVTE & $\varepsilon q \vee$. & $r \leq 7 r$ & $r .17$ \\
\hline 941 & $r \leq r$ & $\varepsilon \wedge \leqslant .1$ & $1 \leqslant \Lambda . \vee$ & $1.0 \mathrm{~V}$ & $r \leqslant r$ & 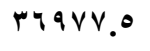 & $\leq \wedge \leq .1$ & rqrq৭.1 & $1 \leqslant \Lambda . V$ & $\Lambda \vee \cdot \Lambda . \nearrow$ & $90 . Y 4$ & 10V0 & $\Delta \vee \cdot \leq \Lambda$ & $\varepsilon \cdot \Delta \wedge r$ & الإجمالي \\
\hline $0 \leqslant . Y$ & $r v . \wedge$ & $r \wedge .0$ & ^. V & Tr.r & $r v . \wedge$ & YIVO & $r \wedge .0$ & IVYI.V & $\wedge . \vee$ & $01 Y . r$ & $00 \wedge q$ & 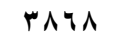 & rror & rrAV & المتوسط \\
\hline & & & إلمنتِج & الزئة & ग) - & ة التسويقية النسبي & (1) & & & ئة & للة) & زَئة ـسعر & (سعر = = & بلني (جما & 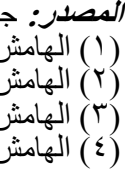 \\
\hline
\end{tabular}


وتوضح دالة الكفاءة التسويقية لمحصول المانجو قبل إضافة

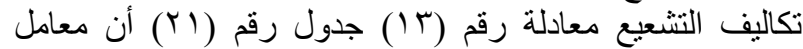

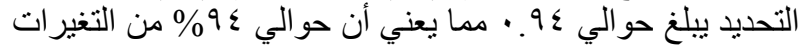

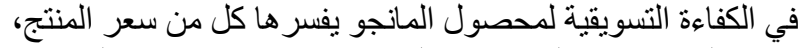

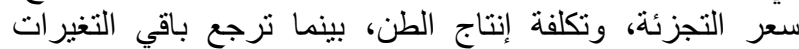
لعو امل أخرى لم تشملها دالة الكفاءة التسويقية. r- العوامل المؤثرة على الكفاءة التسويقية لمحصول المـاتجو قبل وبعد إضافة تكاليف التشعيع:

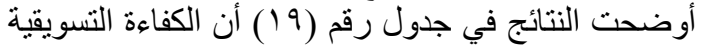

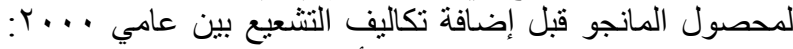

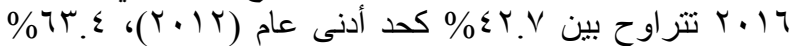

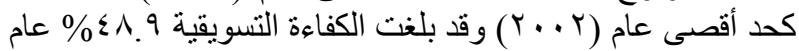

( $($. 17$)$

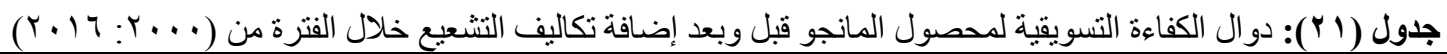

\begin{tabular}{|c|c|c|c|}
\hline $\mathbf{R}^{-2}$ & $\mathbf{F}$ & المعادلة & التغتغير \\
\hline 0.94 & $69.7^{* * *}$ & $\begin{aligned} \mathrm{E}= & 52.26+0.02 \mathrm{P}_{\mathrm{f}}-0.01 \mathrm{P}_{\mathrm{r}}+0.01 \mathrm{C}_{\mathrm{p}} \rightarrow(13) \\
& (25.86)^{* * *}(5.54)^{* * *}(-10.86)^{* * *}(-1.64)^{* * *}\end{aligned}$ & الكفاءة التسويقية \\
\hline 0.55 & $5.2^{* * *}$ & $\begin{aligned} \mathrm{E}=0 \cdot r \wedge+0.01 \mathrm{P}_{\mathrm{f}}-0.007 \mathrm{P}_{\mathrm{r}}+0.0013 \mathrm{C}_{\mathrm{p}} \rightarrow(14) & (29.49)^{* * *}(3.60)^{* * *}(-3.65)^{* * *}(1.01)^{* * *}\end{aligned}$ & بعد إضافة التشعائيفة نكاليفة \\
\hline
\end{tabular}

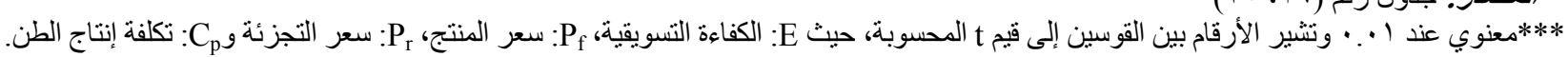

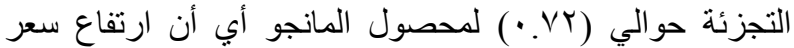

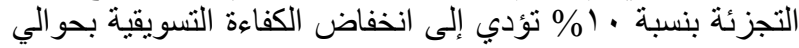
\% \%.Y

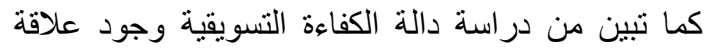
طردية بين كل من الكفاءة التسويقية كمتغير تابع وتكلفة إنتاج الطن

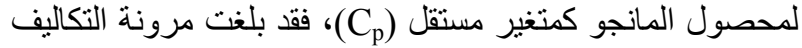
حوالي (0 • . • ) لمحصول الجو كمنغر المانجو.

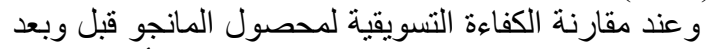

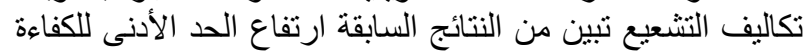

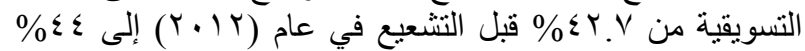

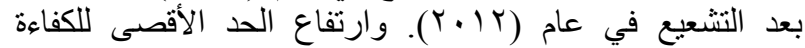

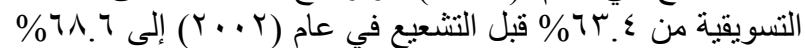

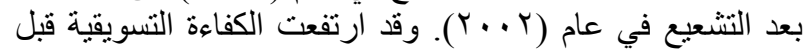

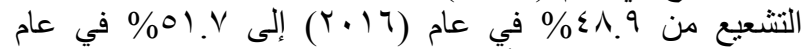

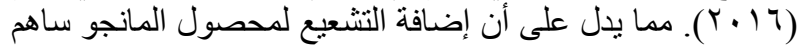

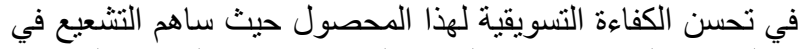

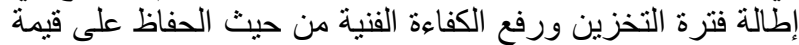

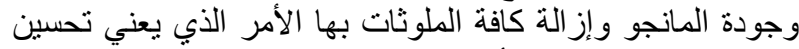
التكاليف التسويقية بنسبة أكبر من التحسين في التكاليف الانتاجية.

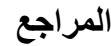

1- إسماعيل، إسماعيل، والقنبيط، محمد (990 (19): (التسويق

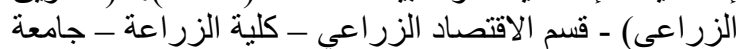

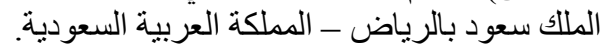

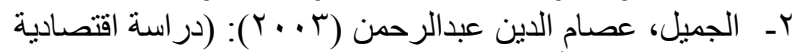

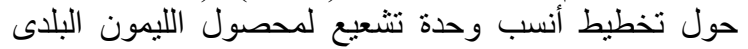

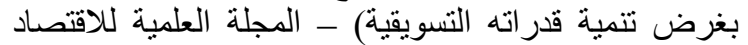
و التجارة. الجميل، عصام الدين عبدالرحمن وميرفت الخطيب (0 . ب):

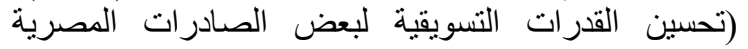

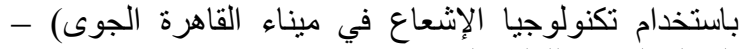

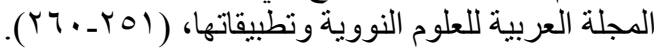

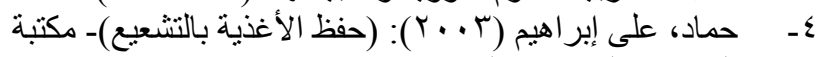

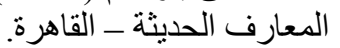

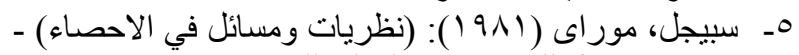
دار ماكجو هيل للنشر - ـدار الدولية للنشر.
حيث تبين من در اسة الكفاءة التسويقية وجود علاقة طردية مؤكدة احصائياً بين كل من الكفاءة التسويقية كمتغير تنابع (E)

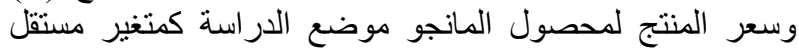

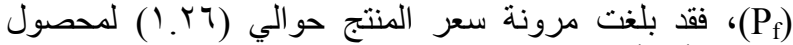

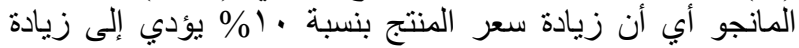

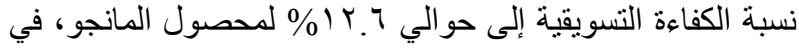

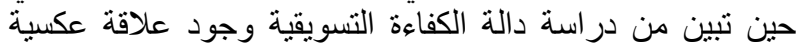
مؤكدة احصائياً بين كل من الكفاءة التسويقية كمنغير تنابع (E)

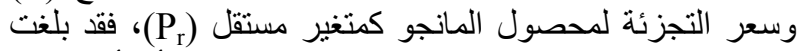

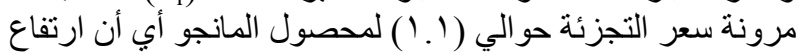

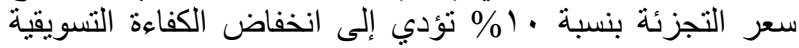

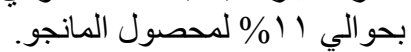

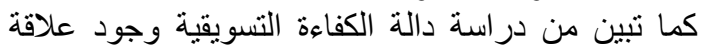

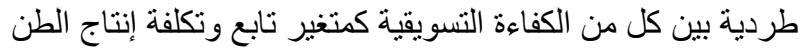

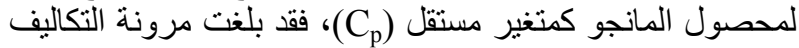

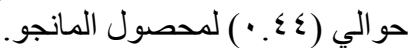

أوضحت النتائج في جدول رقم (Y) (†) أن الكفاءة التسويقية

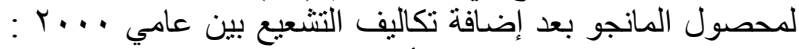

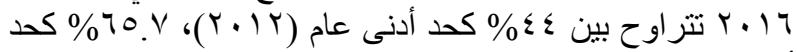

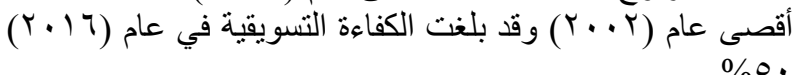

كما توضح دالة الكفاءة التسويقية لمحصول المانجو بعد أنداء

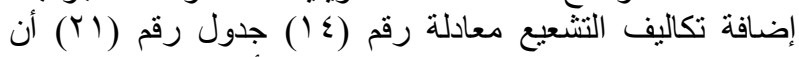

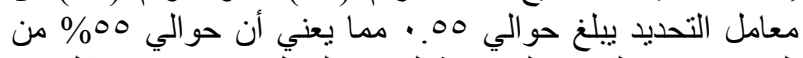

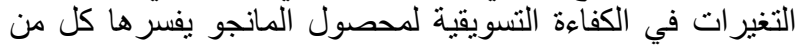

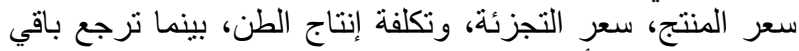

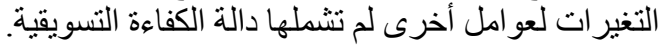

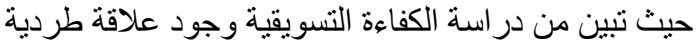
مؤكدة احصائياً بين كل من الكفاءة التسويقية كمنغير تابع (E)

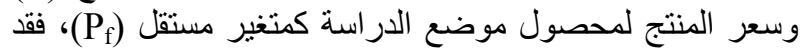

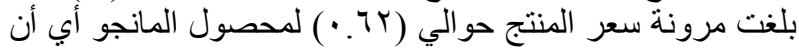

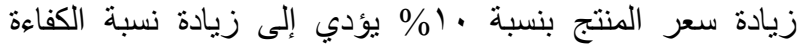

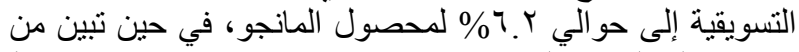
در اسة دالة الكفاءة التسويقية وجود علاقة الكة عكسية مؤكدة احصائياً

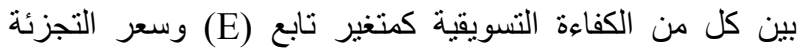
لمحصول المانجو كمتغير مستقل (Prr)، فقد بلغت (E) مرونة سعر 


$$
\begin{aligned}
& \text { الزراعة جامعة القاهرة - جامعة الأزهر - المجلة المصرية }
\end{aligned}
$$

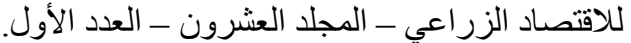

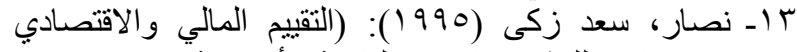

$$
\begin{aligned}
& \text { و الاجتماعي للمشرو عات) - المكتبة الأكاديمية. }
\end{aligned}
$$

14- EFSA (European Food Safety Authority), (2011). Statement summarizing the conclusions and recommendations from the opinions on the safety of irradiation of food adopted by the BIOHAZ and CEF panels, The EFSA J., Vol. 9, no. 4, pp. 2107.

15- Farkas, J. and Mohácsi-Farkas, C., History and future of food irradiation, Food Sci. Technol. 22(2011),121-128.

16- Kume, T.; Furuta, M.; Todoriki, S.; Uenoyama, N. \& Kobayashi, Y. (2009). Status of food irradiation in the world, Radiation Physics and Chemistry, Vol. 78, no. 3, pp. 222-226.

17- Tomlins, K. (2008). Food safety and quality management,

Food

Africa.http://foodafrica.nri.org/safety/safetydis cussions 1.html.

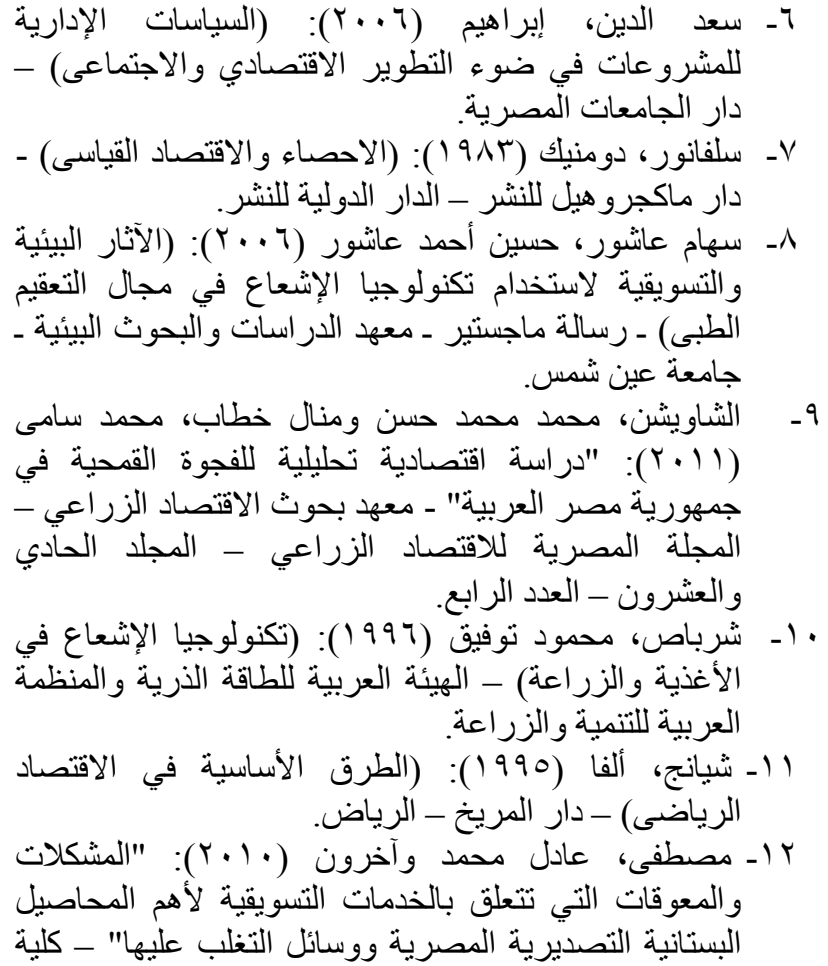

\title{
Marketing Efficiency of some Irradiated Agricultural Crops in Egypt
}

\author{
Shams, S. E. ${ }^{1}$; I. B. Ibrahim ${ }^{1}$; A. A. El Shaer ${ }^{1}$; Essam E. A. El Gamil ${ }^{2}$ and Siham H. A. Ashour ${ }^{2}$
}

${ }^{1}$ Economic Extension and Rural Sociology Department, Faculty of Agriculture, Suez Canal University, Ismaillia, Egypt

${ }^{2}$ Department of Food Irradiation Research - Atomic Energy Authority - National Center for Radiation Research and Technology - Cairo - Egypt

Received: 10/3/2019

\begin{abstract}
This study investigates the use of irradiation technology to improve the marketing efficiency of some agricultural food crops: dates, wheat, maize, potatoes, peanuts, oranges and mango. The factors affecting the marketing efficiency of these agricultural crops were determined using irradiation and a comparative study of marketing efficiency. And after the use of irradiation through the years of 2000: 2016 for agricultural crops under study, where the study showed the increase of the minimum and maximum marketing efficiency after irradiation compared to irradiation, where the contribution of irradiation in reducing the loss and prolong the period Conservation and removal of microbial contaminants and insecticides and fungal food and maintain a fresh image and its value and quality, which contributed to increasing the marketing capabilities of these crops, which means improving the marketing costs greater than the improvement in productivity costs by. Where for example in the date crop the minimum marketing efficiency increased from $8.16 \%$ before irradiation to $40.48 \%$ after irradiation and the maximum marketing efficiency increased from 55.28 before irradiation to 57.54 after irradiation.
\end{abstract}

Keywords: Food losses, financial analysis, national returns, electronic accelerator, time trend 